\title{
Search for chargino-neutralino production using recursive jigsaw reconstruction in final states with two or three charged leptons in proton-proton collisions at $\sqrt{s}=13 \mathrm{TeV}$ with the ATLAS detector
}

\author{
M. Aaboud et al. \\ (ATLAS Collaboration)
}

(Received 7 June 2018; published 19 November 2018)

\begin{abstract}
A search for electroweak production of supersymmetric particles is performed in two-lepton and threelepton final states using recursive jigsaw reconstruction, a technique that assigns reconstructed objects to the most probable hemispheres of the decay trees, allowing one to construct tailored kinematic variables to separate the signal and background. The search uses data collected in 2015 and 2016 by the ATLAS experiment in $\sqrt{s}=13 \mathrm{TeV}$ proton-proton collisions at the CERN Large Hadron Collider corresponding to an integrated luminosity of $36.1 \mathrm{fb}^{-1}$. Chargino-neutralino pair production, with decays via $W / Z$ bosons, is studied in final states involving leptons and jets and missing transverse momentum for scenarios with large and intermediate mass splittings between the parent particle and lightest supersymmetric particle, as well as for the scenario where this mass splitting is close to the mass of the $Z$ boson. The latter case is challenging since the vector bosons are produced with kinematic properties that are similar to those in Standard Model processes. Results are found to be compatible with the Standard Model expectations in the signal regions targeting large and intermediate mass splittings, and chargino-neutralino masses up to $600 \mathrm{GeV}$ are excluded at $95 \%$ confidence level for a massless lightest supersymmetric particle. Excesses of data above the expected background are found in the signal regions targeting low mass splittings, and the largest local excess amounts to 3.0 standard deviations.
\end{abstract}

DOI: 10.1103/PhysRevD.98.092012

\section{INTRODUCTION}

Supersymmetry (SUSY) [1-6] is a generalization of space-time symmetries which predicts new bosonic (fermionic) partners for the fermions (bosons) of the Standard Model (SM). If $R$-parity [7] is conserved, SUSY particles (called sparticles) are produced in pairs and the lightest supersymmetric particle (LSP) is stable and represents a possible dark-matter candidate $[8,9]$. Superpartners of the charged and neutral electroweak (EW) and Higgs bosons mix, producing charginos $\left(\tilde{\chi}_{l}^{ \pm}, l=1,2\right)$ and neutralinos $\left(\tilde{\chi}_{m}^{0}, m=1,2,3,4\right)$, collectively known as electroweakinos. The indices of these particles are ordered by mass in ascending order.

The production cross sections of sparticles at the Large Hadron Collider (LHC) depend both on the type of interaction involved and on the sparticle masses. The

*Full author list given at the end of the article.

Published by the American Physical Society under the terms of the Creative Commons Attribution 4.0 International license. Further distribution of this work must maintain attribution to the author(s) and the published article's title, journal citation, and DOI. Funded by SCOAP . colored sparticles (squarks and gluinos) are produced in strong interactions with significantly larger production cross sections than noncolored sparticles of equal mass, such as the charginos and neutralinos. However, should the masses of gluinos and squarks prove to be out of reach at the LHC, the direct production of charginos and neutralinos could be the dominant sparticle production mode. With searches performed by the ATLAS and CMS Collaborations during LHC Run 2, the exclusion limits on colored-sparticle masses extend up to approximately $2 \mathrm{TeV}$ [10-12], making electroweak production an increasingly promising probe for SUSY signals at the LHC.

This paper presents a search for pair-produced electroweakinos $\left(\tilde{\chi}_{1}^{ \pm} \tilde{\chi}_{2}^{0}\right)$, with each of $\tilde{\chi}_{1}^{ \pm}$and $\tilde{\chi}_{2}^{0}$ decaying to a $\tilde{\chi}_{1}^{0}$ (assumed to be the LSP) and a $W$ or $Z$ gauge boson, respectively, leading to final states with two or three isolated leptons (here taken to be electrons or muons only) which may be accompanied by jets and missing transverse momentum. The analysis uses an integrated luminosity of $36.1 \mathrm{fb}^{-1}$ of proton-proton ( $p p$ ) collision data delivered by the LHC at a center-of-mass energy of $\sqrt{s}=13 \mathrm{TeV}$. The search employs the recursive jigsaw reconstruction (RJR) technique $[13,14]$ in the construction of a suite of complementary discriminating variables. Signal regions are 
defined to probe a wide range of $\tilde{\chi}_{1}^{ \pm} / \tilde{\chi}_{2}^{0}$ (assumed to be mass degenerate) and $\tilde{\chi}_{1}^{0}$ masses, with mass differences $\Delta m=m_{\tilde{\chi}_{1}^{ \pm} / \tilde{\chi}_{2}^{0}}-m_{\tilde{\chi}_{1}^{0}}$ ranging from $\approx 100 \mathrm{GeV}$ to $\approx 600 \mathrm{GeV}$. This search has improved sensitivity to supersymmetric models previously studied by the ATLAS [15-18] and CMS [19-21] Collaborations with the same integrated luminosity, which had expected exclusion sensitivities at 95\% confidence level (C.L.) of $\tilde{\chi}_{1}^{ \pm} / \tilde{\chi}_{2}^{0}$ masses up to 530 and $570 \mathrm{GeV}$, respectively, for a massless LSP.

In a separate search by ATLAS detailed in Ref. [18], where the same SUSY scenarios are considered and the same data set is used, an approach based on conventional variables complements the use of recursive jigsaw variables herein. In both cases, regions are enriched with events containing two or three leptons sensitive to the production of sparticles. In the approach described in Ref. [18], selection criteria are imposed on object momenta, missing transverse momentum and angular parameters to reduce the background and define regions sensitive to signal events. On the other hand, the RJR approach provides a way to reconstruct the event from the detected particles in the presence of kinematic and combinatoric ambiguities by factorizing missing information according to decays and rest frames of intermediate particles. This yields a basis of largely uncorrelated variables that are subsequently used to design the search presented herein. The two different approaches yield event samples that are largely unique and nonoverlapping in the signal regions targeted, with improved sensitivity in the simplified model used to optimize the search. The main SM backgrounds to the search arise from diboson and $Z+$ jet processes.

\section{THE ATLAS DETECTOR}

The ATLAS detector [22] is a multipurpose particle detector with a forward-backward symmetric cylindrical geometry and nearly $4 \pi$ coverage in solid angle. ${ }^{1}$ The inner detector (ID) tracking system consists of silicon pixel and microstrip detectors covering the pseudorapidity region $|\eta|<2.5$, surrounded by a transition radiation tracker, which improves electron identification over the region $|\eta|<2$.0. The innermost pixel layer, the insertable B-layer [23], was added between Run 1 and Run 2 of the LHC, at

\footnotetext{
${ }^{1}$ ATLAS uses a right-handed coordinate system with its origin at the nominal interaction point in the center of the detector. The positive $x$ axis is defined by the direction from the interaction point to the center of the LHC ring, with the positive $y$ axis pointing upwards, while the beam direction defines the $z$ axis. Cylindrical coordinates $(r, \phi)$ are used in the transverse plane, $\phi$ being the azimuthal angle around the $z$ axis. The pseudorapidity $\eta$ is defined in terms of the polar angle $\theta$ by $\eta=-\ln \tan (\theta / 2)$ and the rapidity is defined as $y=(1 / 2) \ln \left[\left(E+p_{z}\right) /\left(E-p_{z}\right)\right]$ where $E$ is the energy and $p_{\mathrm{z}}$ the longitudinal momentum of the object of interest. The transverse momentum $p_{\mathrm{T}}$, the transverse energy $E_{\mathrm{T}}$ and the missing transverse momentum $E_{\mathrm{T}}^{\text {miss }}$ are defined in the $x-y$ plane unless stated otherwise.
}

an average radius of $33 \mathrm{~mm}$ around a new, narrower and thinner beam pipe. The ID is surrounded by a thin superconducting solenoid providing an axial $2 \mathrm{~T}$ magnetic field and by a fine-granularity lead/liquid-argon (LAr) electromagnetic (EM) calorimeter covering $|\eta|<3.2$. A steel/ scintillator-tile hadronic calorimeter provides coverage in the central pseudorapidity range $(|\eta|<1.7)$. The end cap and forward regions are instrumented with LAr calorimeters for both EM and hadronic energy measurements up to $|\eta|=4$.9. The muon spectrometer with an air-core toroid magnet system surrounds the calorimeters. Three layers of high-precision tracking chambers provide coverage in the range $|\eta|<2.7$, while dedicated chambers allow triggering in the region $|\eta|<2.4$.

The trigger system [24] consists of two levels. The first level is a hardware-based system and uses a subset of the detector information. The second is a software-based system called the high-level trigger which runs offline reconstruction and calibration software, reducing the event rate to about $1 \mathrm{kHz}$.

\section{DATA AND MONTE CARLO SAMPLES}

The data were collected by the ATLAS detector during 2015 with a peak instantaneous luminosity of $L=$ $5.2 \times 10^{33} \mathrm{~cm}^{-2} \mathrm{~s}^{-1}$, and during 2016 with a maximum of $L=1.37 \times 10^{34} \mathrm{~cm}^{-2} \mathrm{~s}^{-1}$. The mean number of $p p$ interactions per bunch crossing (pileup) in the data set was $\langle\mu\rangle=14$ in 2015 and $\langle\mu\rangle=24$ in 2016. Application of beam, detector and data-quality criteria resulted in a total integrated luminosity of $36.1 \mathrm{fb}^{-1}$. The uncertainty in the integrated luminosity is $\pm 2.1 \%$. It is derived, following a methodology similar to that detailed in Ref. [25], from a calibration of the luminosity scale using $x-y$ beam-separation scans performed in August 2015 and May 2016.

A set of Monte Carlo (MC) background and signal samples of simulated events is used to optimize the selection criteria and assess the sensitivity to specific SUSY signal models. Where applicable, the MC samples are used in the background estimation as well.

The production of $Z$ bosons in association with jets [26] was performed with the SHERPA 2.2.1 generator [27]. The NNPDF3.0NNLO [28] parton distribution function (PDF) was used in conjunction with dedicated parton shower tuning developed by the SHERPA authors. The matrix elements (ME) were calculated for up to two partons at next-to-leading order (NLO) and with up to two additional partons at leading order (LO) using the COMIX [29] and OPEN LOOPS [30] matrix-element generators, and merged with the SHERPA parton shower (PS) [31] using the $\mathrm{ME}+\mathrm{PS} @$ NLO prescription [32]. For MC closure studies of the data-driven $Z+$ jets background estimate (described in Sec. VIII A), $\gamma+$ jets events were generated at LO with up to four additional partons using the SHERPA 2.1.1 generator with CT10 [33] PDF set. 
The Powheg-Box v2 [34] generator was used for the generation of $t \bar{t}$ and single-top-quark processes in the $W t$ and $s$-channels [35], while $t$-channel single-top production was modeled using PowHEG-Box v1 [36]. For the latter process, the decay of the top quark was simulated using MADSPIN [37] preserving all spin correlations. For all processes the CT10 [33] PDF set was used for the matrix element, while the parton shower, fragmentation, and the underlying event were generated using PYTHIA 6.428 [38] with the CTEQ6L1 [39] PDF set and a set of tuned parameters called the Perugia 2012 tune [40]. The topquark mass in all samples was set to $172.5 \mathrm{GeV}$. The $t \bar{t}$ and the $W t$-channel single-top events were normalized to cross sections calculated at next-to-next-to-leading order plus next-to-next-to-leading-logarithm (NNLO + NNLL) [41-44] accuracy, while $s$ - and $t$-channel single-top-quark events were normalized to the NLO cross sections $[45,46]$. The production of $Z t$ events was generated with the MG5_aMC@NLO 2.2.1 [47] generator at LO with the CTEQ6L1 PDF set.

The MG5_aMC@NLO 2.2.2 (2.2.3 for $\left.t \bar{t}+Z / \gamma^{*}\right)$ generator at LO, interfaced to the PYTHIA 8.186 [48] partonshower model, was used for the generation of $t \bar{t}+\mathrm{EW}$ processes $(t \bar{t}+W / Z / W W)[49]$, with up to two $[t \bar{t}+W$, $t \bar{t}+Z(\rightarrow \nu \nu / q q)]$, one $\left[t \bar{t}+Z(\rightarrow \ell \ell)^{2}\right]$ or no $(t \bar{t}+W W)$ extra partons included in the matrix element. The events were normalized to their respective NLO cross sections [50,51].

Diboson processes $(W W, W Z, Z Z)[52]$ were simulated using the SHERPA 2.2.1 generator and contain off-shell contributions. For processes with four charged leptons $(4 \ell)$, three charged leptons and a neutrino $(3 \ell+1 \nu)$ or two charged leptons and two neutrinos $(2 \ell+2 \nu)$, the matrix elements contain all diagrams with four electroweak couplings, and were calculated for up to one $(4 \ell, 2 \ell+2 \nu)$ or no extra partons $(3 \ell+1 \nu)$ at NLO. All diboson samples were also simulated with up to three additional partons at LO using the COMIX and OpENLOOPS matrix-element generators, and were merged with the SHERPA parton shower using the ME + PS@ NLO prescription. The diboson events were normalized to their NLO cross sections $[53,54]$. Additional MC simulation samples of events with a leptonically decaying vector boson and photon, $V \gamma$, were generated at LO using SHERPA 2.1.1 [27]. Matrix elements including all diagrams with three electroweak couplings were calculated with up to three partons at LO and merged with the SHERPA parton shower [55] according to the ME+PS@LO prescription [56]. The CT10 PDF set is used in conjunction with dedicated parton shower tuning developed by the SHERPA authors.

\footnotetext{
${ }^{2}$ The letter $\ell$ stands for the charged leptons (electrons, muons and taus). While the contributions from tau leptons are included in all the Monte Carlo samples, in the next sections the symbol $\ell$ refers to electrons and muons only.
}

Triboson processes $(W W W, W W Z, W Z Z$ and $Z Z Z)$ were simulated with the SHERPA 2.2.1 generator with matrix elements calculated at LO with up to one additional parton. The triboson events were normalized to their LO cross sections [57].

Higgs-boson production processes (including gluongluon fusion, associated vector-boson production, $\mathrm{VH},{ }^{3}$ and vector-boson fusion, VBF) were generated using POWHEG v2 [35]+PyTHIA 8.186 and normalized to cross sections calculated at NNLO with soft gluon emission effects added at NNLL accuracy, while $t \bar{t} H$ events were produced using aMC@NLO 2.2.2+ HeRwIG 2.7.1 [58] and normalized to the NLO cross section [59]. All samples assume a Higgs boson mass of $125 \mathrm{GeV}$.

Simplified models [60] are defined by an effective Lagrangian describing the interactions of a small number of new particles, assuming one production process and one decay channel with a $100 \%$ branching ratio. Specifically, the SUSY production modes considered in this paper are studied in the context of simplified models, assuming wino-like chargino-neutralino production with decays via Standard Model $W$ and $Z$ gauge bosons and a bino-like LSP, leading to two- and three-lepton final states. As illustrated in Fig. 1, two scenarios are considered: one where the $W$ boson decays leptonically resulting in a threelepton plus missing-transverse-momentum $\left(E_{\mathrm{T}}^{\text {miss }}\right)$ final state [Fig. 1(a)], and one where the $W$ boson decays hadronically, yielding two leptons with same flavor and opposite-sign charge plus two jets plus $E_{\mathrm{T}}^{\text {miss }}$ in the final state, as in Fig. 1(b). Figures 1(c) and 1(d) show the diagrams where the $\tilde{\chi}_{1}^{ \pm} \tilde{\chi}_{2}^{0}$ system is produced in association with an initial state radiation (ISR) jet leading again to three-lepton and two-lepton final states.

The MC signal samples were generated from leadingorder matrix elements with up to two extra partons using MADGRAPH v2.2.3 [61] interfaced to PYTHIA version 8.186, with the A14 parameter tune [62], for the modeling of the SUSY decay chain, parton showering, hadronization and the description of the underlying event. Parton luminosities were provided by the NNPDF23LO PDF set [33]. Jetparton matching follows the CKKW-L prescription [63], with a matching scale set to one quarter of the $\tilde{\chi}_{1}^{ \pm} / \tilde{\chi}_{2}^{0}$ mass. Signal cross sections were calculated at NLO in the strong coupling constant, with soft gluon emission effects added at next-to-leading-logarithm (NLL) accuracy [64-68]. The nominal cross section and the uncertainty were taken from an envelope of cross-section predictions using different PDF sets and factorization and renormalization scales, as described in Ref. [69]. For $\tilde{\chi}_{1}^{ \pm}$and $\tilde{\chi}_{2}^{0}$ with a mass of $500 \mathrm{GeV}$, the production cross section is $46 \pm 4 \mathrm{fb}$ at $\sqrt{s}=13 \mathrm{TeV}$.

\footnotetext{
${ }^{3}$ The letter $V$ represents the $W$ or $Z$ gauge boson.
} 


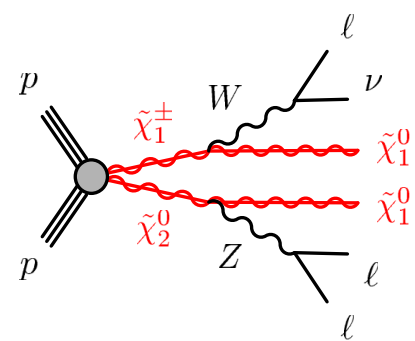

(a)

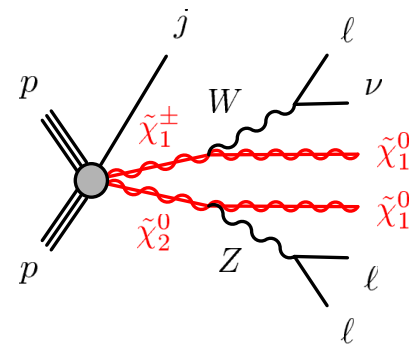

(c)

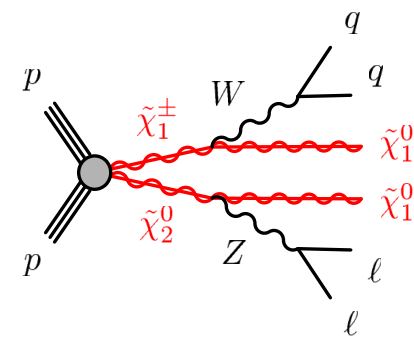

(b)

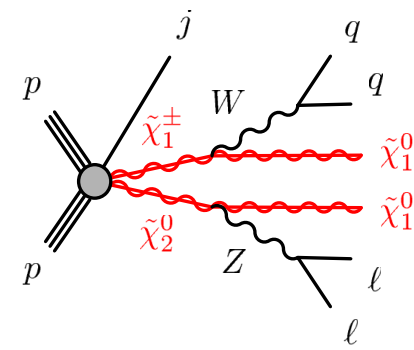

(d)
FIG. 1. Diagrams for the physics scenarios studied in this paper: (a) $\tilde{\chi}_{1}^{ \pm} \tilde{\chi}_{2}^{0}$ with decays via leptonically decaying $W$ and $Z$ bosons, (b) $\tilde{\chi}_{1}^{ \pm} \tilde{\chi}_{2}^{0}$ with decays to two-lepton plus two-jet plus $E_{\mathrm{T}}^{\text {miss }}$ final states through a hadronically decaying $W$ boson and a leptonically decaying $Z$ boson, (c) $\tilde{\chi}_{1}^{ \pm} \tilde{\chi}_{2}^{0}$ production in association with an initial state radiation jet (labeled " $j$ " in the figure) with decays via leptonically decaying $W$ and $Z$ bosons and (d) $\tilde{\chi}_{1}^{ \pm} \tilde{\chi}_{2}^{0}$ production in association with an initial state radiation jet with decays to twolepton plus two-jet plus $E_{\mathrm{T}}^{\text {miss }}$ final states through a hadronically decaying $W$ boson and a leptonically decaying $Z$ boson.

A summary of the SUSY signals and the SM background processes together with the MC generators, cross-section calculation orders in $\alpha_{\mathrm{s}}$, PDFs, parton shower and parameter tunes used is given in Table I.
The EvTGEN v1.2.0 program [70] was used to model the decays of $b$ - and $c$-hadrons in the SM background samples except for those produced with SHERPA. All simulated events were overlaid with multiple $p p$ collisions simulated with the soft QCD processes of PYTHIA 8.186 using the A2 tune [71] and the MSTW2008LO parton distribution functions [72]. The MC samples were generated with a variable number of additional $p p$ interactions in the same and neighboring bunch crossings, and were reweighted to match the distribution of the mean number of interactions observed in data.

For all SM background samples the response of the detector to particles was modeled with a full ATLAS detector simulation [73] based on GEANT4 [74]. Signal samples were prepared using a fast simulation based on a parametrization of the performance of the ATLAS electromagnetic and hadronic calorimeters and on GEANT4 elsewhere.

\section{OBJECT RECONSTRUCTION AND IDENTIFICATION}

The reconstructed primary vertex of the event is required to be consistent with the luminous region and to have at least two associated tracks with $p_{\mathrm{T}}>400 \mathrm{MeV}$. When more than one such vertex is found, the vertex with the largest $\sum p_{T}^{2}$ of the associated tracks is chosen.

Two different classes of reconstructed lepton candidates (electrons or muons) are used in the analysis, labeled baseline and high-purity in the following. When selecting samples for the search, events must contain a minimum of two baseline electrons or muons.

Baseline muon candidates are formed by combining information from the muon spectrometer and ID as

TABLE I. The SUSY signals and the Standard Model background Monte Carlo samples used in this paper. The generators, the order in $\alpha_{\mathrm{S}}$ of cross-section calculations used for yield normalization, PDF sets, parton showers and parameter tunes used for the underlying event are shown.

\begin{tabular}{|c|c|c|c|c|c|}
\hline Physics process & Generator & Cross-section normalization & PDF set & Parton shower & Tune \\
\hline SUSY processes & MADGRAPH v2.2.3 & $\mathrm{NLO}+\mathrm{NLL}$ & NNPDF2.3LO & PYTHIA 8.186 & A14 \\
\hline$Z / \gamma^{*}(\rightarrow \ell \bar{\ell})+$ jets & SHERPA 2.2.1 & NNLO & NNPDF3.0NNLO & SHERPA & SHERPA default \\
\hline$\gamma+$ jets & SHERPA 2.1.1 & LO & CT10 & SHERPA & SHERPA default \\
\hline$H(\rightarrow \tau \tau), H(\rightarrow W W)$ & POWHEG-BOX v2 & NLO & CTEQ6L1 & PYTHIA 8.186 & A14 \\
\hline$H W, H Z$ & MG5_aMC@NLO 2.2.2 & NLO & NNPDF2.3LO & PYTHIA 8.186 & A14 \\
\hline$t \bar{t}+H$ & MG5_aMC@NLO 2.2.2 & NLO & CTEQ6L1 & HERWIG 2.7.1 & A14 \\
\hline$t \bar{t}$ & PowHEG-Box v2 & NNLO + NNLL & CT10 & PYTHIA 6.428 & Perugia2012 \\
\hline Single top ( $W t$-channel $)$ & POWHEG-Box v2 & NNLO + NNLL & CT10 & РYTHIA 6.428 & Perugia2012 \\
\hline Single top ( $s$-channel) & POWHEG-BOX v2 & NLO & CT10 & PYTHIA 6.428 & Perugia2012 \\
\hline Single top ( $t$-channel) & POWHEG-BOX v1 & NLO & CT10f4 & PYTHIA 6.428 & Perugia2012 \\
\hline Single top ( $Z t$-channel) & MG5_aMC@NLO 2.2.1 & LO & CTEQ6L1 & PYTHIA 6.428 & Perugia2012 \\
\hline$t \bar{t}+W / W W$ & MG5_aMC@NLO 2.2.2 & NLO & NNPDF2.3LO & РYTHIA 8.186 & A14 \\
\hline$t \bar{t}+Z$ & MG5_aMC@NLO 2.2.3 & NLO & NNPDF2.3LO & PYTHIA 8.186 & A14 \\
\hline$W W, W Z, Z Z$ & SHERPA 2.2 .1 & NLO & NNPDF30NNLO & SHERPA & SHERPA default \\
\hline$V \gamma$ & SHERPA 2.1.1 & LO & CT10 & SHERPA & SHERPA default \\
\hline Triboson & SHERPA 2.2 .1 & NLO & NNPDF30NNLO & SHERPA & SHERPA default \\
\hline
\end{tabular}


described in Ref. [75], must pass the medium identification requirements defined therein, and have $p_{\mathrm{T}}>10 \mathrm{GeV}$ and $|\eta|<2.7$. High-purity muon candidates must additionally have $|\eta|<2.4$, the significance of the transverse impact parameter relative to the primary vertex $\left|d_{0}^{\mathrm{PV}}\right| / \sigma\left(d_{0}^{\mathrm{PV}}\right)<3$, and the longitudinal impact parameter relative to the primary vertex $\left|z_{0}^{\mathrm{PV}} \sin \theta\right|<0.5 \mathrm{~mm}$. Furthermore, highpurity candidates must satisfy the 'GradientLoose' isolation requirements described in Ref. [75], which rely on tracking-based and calorimeter-based variables and implement a set of $\eta$ - and $p_{\mathrm{T}}$-dependent criteria. The highest- $p_{\mathrm{T}}$ (leading) high-purity muon is also required to have $p_{\mathrm{T}}>25 \mathrm{GeV}$.

Baseline electron candidates are reconstructed from an isolated electromagnetic calorimeter energy deposit matched to an ID track. They are required to have $p_{\mathrm{T}}>10 \mathrm{GeV},|\eta|<2.47$, and to satisfy a set of quality criteria similar to the loose likelihood-based identification criteria described in Ref. [76], but including a requirement of a B-layer hit. High-purity electron candidates additionally must satisfy medium $L H$ selection criteria described in Ref. [76]. They are also required to have $\left|d_{0}^{\mathrm{PV}}\right| / \sigma\left(d_{0}^{\mathrm{PV}}\right)<5$, $\left|z_{0}^{\mathrm{PV}} \sin \theta\right|<0.5 \mathrm{~mm}$, and to satisfy isolation requirements that are the same as those applied to high-purity muons [76]. The leading high-purity electron is also required to have $p_{\mathrm{T}}>25 \mathrm{GeV}$.

Jet candidates are reconstructed using the anti- $k_{t}$ jet clustering algorithm [77-79] with a jet radius parameter of 0.4 starting from clusters of calorimeter cells [80]. The jets are corrected for energy from pileup using the method described in Ref. [81]: a contribution equal to the product of the jet area and the median energy density of the event is subtracted from the jet energy [82]. Further corrections, referred to as the jet energy scale corrections, are derived from MC simulation and data and are used to calibrate the average energies of jets to the scale of their constituent particles [83]. In order to reduce the number of jets originating from pileup, a significant fraction of the tracks associated with each jet must have an origin compatible with the primary vertex, as defined by the jet vertex tagger (JVT) output [84]. Only corrected jet candidates with $p_{\mathrm{T}}>$ $20 \mathrm{GeV}$ and $|\eta|<4.5$ are retained. High-purity jets are defined with the tighter requirement $|\eta|<2.4$. The chosen requirement corresponds to the medium working point of the JVT and is only applied to jets with $p_{\mathrm{T}}<60 \mathrm{GeV}$ and $|\eta|<2.4$. This requirement reduces jets from pileup to $1 \%$ with an efficiency for pure hard-scatter jets of $92 \%$.

An algorithm based on boosted decision trees, MV2c10 $[85,86]$, is used to identify jets containing a $b$-hadron ( $b$-jets), with an operating point corresponding to an efficiency of $77 \%$, and rejection factors of 134 for lightquark and gluon jets and 6 for charm jets [86], for reconstructed jets with $p_{\mathrm{T}}>20 \mathrm{GeV}$ and $|\eta|<2.5$ in simulated $t \bar{t}$ events. Candidate $b$-tagged jets are required to have $p_{\mathrm{T}}>20 \mathrm{GeV}$ and $|\eta|<2.4$.
After the selection requirements described above, ambiguities between candidate jets with $|\eta|<4.5$ and baseline leptons are resolved as follows:

(1) Any electron sharing an ID track with a muon is removed.

(2) If a $b$-tagged jet (identified using the $85 \%$ efficiency working point of the MV2c10 algorithm) is within $\Delta R \equiv \sqrt{(\Delta y)^{2}+(\Delta \phi)^{2}}=0.2$ of an electron candidate, the electron is rejected, as it is likely to originate from a semileptonic $b$-hadron decay; otherwise, if a non- $b$-tagged jet is within $\Delta R=$ 0.2 of an electron candidate then the electron is kept and the jet is discarded as it is likely to be due to the electron-induced shower.

(3) Electrons within $\Delta R=0.4$ of a remaining jet candidate are discarded, to suppress electrons from semileptonic decays of $c$ - and $b$-hadrons.

(4) Jets with fewer than three associated tracks that have a nearby muon that carries a significant fraction of the transverse momentum of the jet $\left(p_{\mathrm{T}}^{\mu}>0.7 \sum p_{\mathrm{T}}^{\text {jet tracks }}\right.$, where ${p_{\mathrm{T}}}^{\mu}$ and $p_{\mathrm{T}}^{\text {jet tracks }}$ are the transverse momenta of the muon and the tracks associated with the jet, respectively) are discarded either if the candidate muon is within $\Delta R=0.2$ or if the muon is matched to a track associated with the jet.

(5) Muons within $\Delta R=0.4$ of a remaining jet candidate are discarded to suppress muons from semileptonic decays of $c$ - and $b$-hadrons.

The events used by the searches described in this paper are selected using high-purity leptons and jets with a trigger logic that accepts events with either two electrons, two muons or an electron plus a muon. The trigger-level requirements on the $p_{\mathrm{T}}$ of the leptons involved in the trigger decision (the $p_{\mathrm{T}}$ thresholds range between 8 and $22 \mathrm{GeV}$ ) are looser than those applied offline to ensure that trigger efficiencies remain high and are constant in the relevant phase space.

Events containing a photon and jets are used to estimate the $Z / \gamma^{*}+$ jets background in the $2 \ell+$ jets channel. These events are selected with a set of prescaled single-photon triggers with $p_{\mathrm{T}}$ thresholds in the range 35-100 GeV and an unprescaled single-photon trigger with threshold $p_{\mathrm{T}}>140 \mathrm{GeV}$. High-purity photons must have $p_{\mathrm{T}}>$ $37 \mathrm{GeV}$ to be on the efficiency plateau of the lowestthreshold single-photon trigger and satisfy a tight identification requirement and $p_{\mathrm{T}}$-dependent requirements on both track- and calorimeter-based isolation [87]. The $\gamma+$ jets control sample, used for the data-driven $Z+$ jets background estimate described in Sec. VIII A, makes use of high-purity photons. The ambiguities between candidate photons, jets and leptons are resolved by applying the following two requirements:

(1) Photons are removed if they reside within $\Delta R=0.4$ of a baseline electron or muon. 
(2) Any jet within $\Delta R=0.4$ of any remaining photon is discarded.

The measurement of the missing transverse momentum vector $\vec{p}_{\mathrm{T}}^{\text {miss }}$ (and its magnitude $E_{\mathrm{T}}^{\text {miss}}$ ) is based on the calibrated transverse momenta of all electron, photon, muon and jet candidates and all tracks originating from the primary vertex and not associated with such objects [88]. The missing transverse momentum is the negative of the vector sum of the object momenta.

\section{ANALYSIS STRATEGY AND BACKGROUND PREDICTION}

To search for a possible signal, selection criteria are defined to enhance the expected signal yield relative to the SM background. Signal regions (SRs) are designed using the MC simulation for both SUSY signals and the SM background processes, before looking at the data in the relevant phase space. They are optimized to maximize the expected sensitivity for the exclusion of each model considered. To estimate the SM backgrounds in an accurate and robust fashion, corresponding control regions (CRs) are defined for each of the signal regions. They are chosen to be orthogonal to the SR selections in order to provide independent data samples enriched in particular backgrounds, and are used to normalize the background MC simulation. The CR selections are optimized to have negligible SUSY signal contamination for the models near the LHC Run 1 excluded region's boundary [17], while minimizing the systematic uncertainties arising from the extrapolation of the CR event yields to estimate backgrounds in the SR. Cross-checks of the background estimates are performed with data in several validation regions (VRs) selected with requirements such that these regions do not overlap with the CR and SR selections, and also have a low expected signal contamination.

To extract the final results, three different classes of likelihood fits are employed, denoted background-only, model-independent and model-dependent fits, using the HistFitter framework [89]. The fits are performed using the total number of events in each region. To obtain a set of background predictions that are independent of the observations in the SRs, the fit can be configured to use only the CRs to constrain the fit parameters: the SR bins are removed from the likelihood and any potential signal contribution is neglected everywhere. This fit configuration is referred to as the background-only fit. The scale factors representing the normalizations of background components relative to $\mathrm{MC}$ predictions are determined in the fit to all the CRs associated with an SR. This is most notably the case for diboson production since it is the dominant background in several SRs. The expected backgrounds in an SR are based on the yields predicted by simulation, corrected by the scale factors derived from the fit. A dedicated datadriven method is used to estimate the $Z+$ jets background yield for the two lepton regions. The systematic and MC statistical uncertainties are included in the fit as nuisance parameters that are constrained by Gaussian distributions with widths corresponding to the sizes of the uncertainties considered and by Poisson distributions, respectively. The background-only fit results are also used to estimate the background event yields in the VRs.

A model-independent fit is used to quantify the level of agreement between background predictions and observed yields and to quantify the number of possible beyond the Standard Model (BSM) signal events in each SR. This fit proceeds in the same way as the background-only fit, except that the number of observed events in the SR is added as an input to the fit, and an additional parameter for the BSM signal strength, constrained to be non-negative, is included. The observed and expected upper limits at 95\% confidence level (C.L.) on the number of events from BSM phenomena for each signal region $\left(S_{\mathrm{obs}}^{95}\right.$ and $\left.S_{\mathrm{exp}}^{95}\right)$ are derived using the $\mathrm{CL}_{\mathrm{s}}$ prescription [90], neglecting any possible signal contamination in the CRs. These limits, when normalized by the integrated luminosity of the data sample, may be interpreted as upper limits on the visible cross section of BSM processes $\left(\langle\epsilon \sigma\rangle_{\text {obs }}^{95}\right)$, where the visible cross section is defined as the product of production crosssection, acceptance and efficiency. The model-independent fit is also used to compute the one-sided $p$-value of the background-only hypothesis $\left(p_{0}\right)$, which quantifies the statistical significance of an excess; $p_{0}$ cannot exceed 0.5.

Finally, a model-dependent fit is used to set exclusion limits on the signal cross sections for specific SUSY models. Such a fit proceeds in the same way as the model-independent fit, except that the yields in both the SRs and the CRs are taken into account. Signal-yield systematic uncertainties due to detector effects and the theoretical uncertainties in the signal acceptance are included in the fit. Correlations between signal and background systematic uncertainties are taken into account where appropriate. Limits on the signal cross section are then mapped into limits on sparticle masses in the twodimensional simplified-model planes.

\section{THE RECURSIVE JIGSAW RECONSTRUCTION TECHNIQUE}

The RJR technique $[13,14]$ is a method for decomposing measured properties event by event to provide a basis of kinematic variables. This is achieved by approximating the rest frames of intermediate particle states in each event. This reconstructed view of the event gives rise to a natural basis of kinematic observables, calculated by evaluating the momentum and energy of different objects in these reference frames. Background processes are reduced by testing whether each event exhibits the anticipated properties of the imposed decay tree under investigation while only applying minimal selection criteria on visible object momenta and missing momenta. The RJR technique is described in detail 
in Refs. [13,14] and has been used in previous ATLAS searches [10,91,92].

Electrons, muons, hadronic jets and $\vec{p}_{\mathrm{T}}^{\text {miss }}$ (as defined in Sec. IV) are used as input to the RJR algorithm. Motivated by searches for pair-production of sparticles in $R$-parityconserving models, a decay tree is constructed following the canonical process in Fig. 2(a), for the $2 \ell$ [Fig. 2(b)] and $3 \ell$ [Fig. 2(c)] search regions, used in the analysis of events. Each event is evaluated as if two sparticles (labeled PP) were produced, assigned to two hemispheres $\left(\mathrm{P}_{\mathrm{a}}\right.$ and $\left.\mathrm{P}_{\mathrm{b}}\right)$ and then decayed to the particles observed in the detector with $\mathrm{V}$ denoting visible objects and I invisible objects. The benchmark signal models probed in this search give rise to signal events with at least two weakly interacting particles associated with two systems of invisible particles (shown in green), the respective children of the initially produced sparticles. For the $2 \ell$ channel the lepton pair must be associated with the same visible collection, similarly for the jets, while for the $3 e$ channel the opposite-charge,

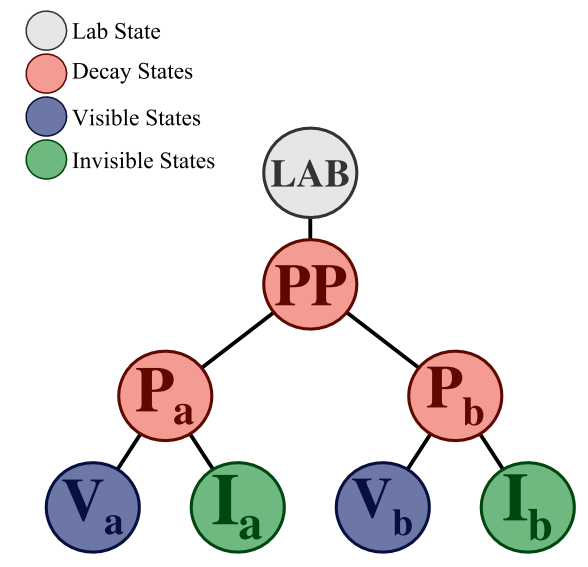

(a)

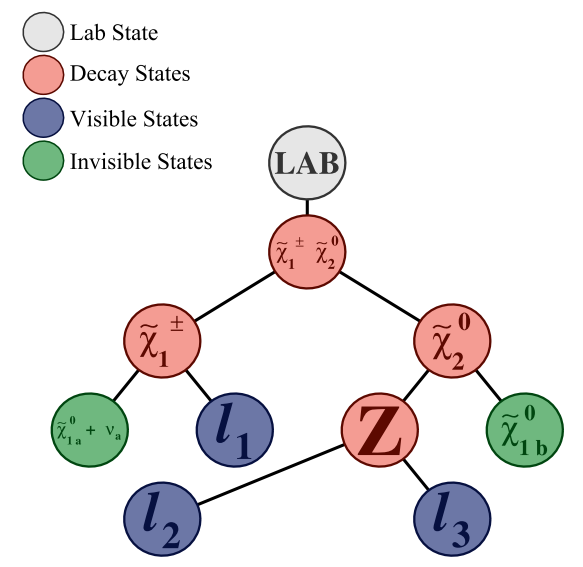

(c) same-flavor pair most consistent with the Z-boson mass is selected as one visible collection, with the unpaired lepton being assigned to the opposite hemisphere (the $Z$ boson being associated with $\mathrm{V}_{\mathrm{b}}$, and the unpaired lepton with $\mathrm{V}_{\mathrm{a}}$ ).

After partitioning the visible objects, the remaining unknowns in the event are associated with the two collections of invisible particles: their masses, longitudinal momenta and information about how the two groups contribute to the $\vec{p}_{\mathrm{T}}^{\text {miss }}$. The RJR algorithm determines these unknowns by identifying the smallest Lorentz invariant function of the visible particles' four vectors that ensures the invisible particle mass estimators remain non-negative [14]. In each of these newly constructed rest frames, all relevant momenta are defined and can be used to construct a set of variables such as multi-object invariant masses and angles between objects. The primary energyscale-sensitive observables used in the search presented here are a suite of variables denoted by $H$. As shown in Eq. (1), the $H$ variables are constructed using different

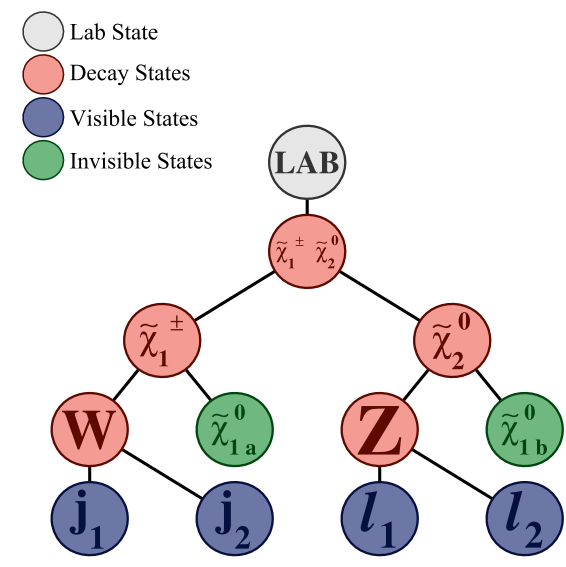

(b)

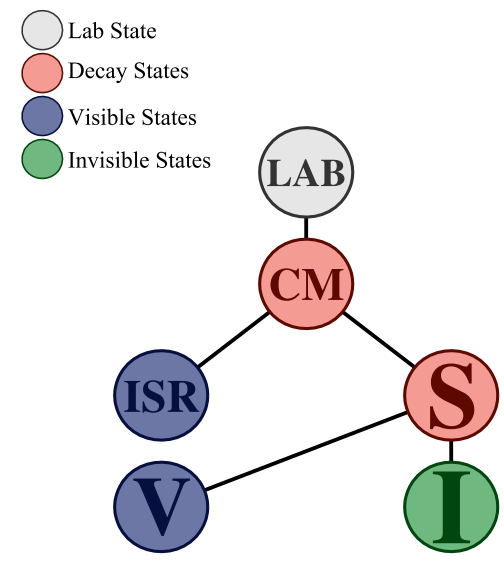

(d)

FIG. 2. (a) The "standard" decay tree applied to pair-produced sparticles ("parent" objects), P, decaying to visible states "V" and invisible states "I." (b) Decay trees for the $2 \ell+2$ jets final state and (c) $3 \ell$ final state. (d) The "compressed" decay tree. CM denotes the center-of-mass frame. A signal sparticle system $\mathrm{S}$ decaying to a set of visible momenta $\mathrm{V}$ and invisible momentum I recoils from a jet-radiation system ISR. 
combinations of object momenta, including contributions from the invisible four-momenta, and are not necessarily evaluated in the lab frame, nor only in the transverse plane,

$$
H_{n, m}^{\mathrm{F}}=\sum_{i=1}^{n}\left|\vec{p}_{\mathrm{vis}, i}^{\mathrm{F}}\right|+\sum_{j=1}^{m}\left|\vec{p}_{\mathrm{inv}, j}^{\mathrm{F}}\right| .
$$

The $H$ variables are labeled with a superscript $\mathrm{F}$ and two subscripts $n$ and $m, H_{n, m}^{\mathrm{F}}$. The F represents the rest frame in which the momenta are evaluated. In this analysis, this may be the lab frame, the proxy for the sparticle-sparticle frame PP, or the proxy for the rest frame of an individual sparticle, P. The subscripts $n$ and $m$ represent the number of visible and invisible momentum vectors considered, respectively. For events with fewer than $n$ visible objects, the sum only runs over the available momenta. Only the leading $n-n_{\ell}$ jets are considered, where $n_{\ell}$ is the number of reconstructed leptons in the event. An additional subscript " $T$ " denotes a transverse version of the variable, where the transverse plane is defined in a frame $\mathrm{F}$ as follows: the Lorentz transformation relating $\mathrm{F}$ to the lab frame is decomposed into a boost along the beam axis, followed by a subsequent transverse boost. The transverse plane is defined to be perpendicular to the longitudinal boost. In practice, this is the plane transverse to the beam line.

The following variables are used in the definition of the signal regions. The value of $n$ differs for the case of events with a leptonic $W$ decay where there are three visible objects and hence $n=3$, and for events with a hadronic $W$ decay where there are four visible objects, and thus $n=4$.

(i) $H_{n, 1}^{\mathrm{PP}}$ : scale variable as described above. Behaves similarly to the effective mass, $m_{\text {eff }}$ (defined as the scalar sum of the transverse momenta of the visible objects and $E_{\mathrm{T}}^{\text {miss }}$ ), used in previous ATLAS SUSY searches.

(ii) $H_{1,1}^{\mathrm{PP}} / H_{4,1}^{\mathrm{PP}}$ : provides additional information in testing the balance of the two scale variables. This provides excellent discrimination against unbalanced events where the large scale is dominated by a particular object $p_{\mathrm{T}}$ or by large $E_{\mathrm{T}}^{\text {miss }}$. Behaves similarly to the $E_{\mathrm{T}}^{\text {miss }} / m_{\text {eff }}$. Utilized solely in the $2 \ell$ low mass signal region to mitigate the effects of $Z+$ jets backgrounds, in cases where one high $p_{\mathrm{T}}$ jet dominates.

(iii) $p_{\mathrm{TPP}}^{\mathrm{lab}} /\left(p_{\mathrm{TPP}}^{\mathrm{lab}}+H_{\mathrm{T} n, 1}^{\mathrm{PP}}\right)$ : compares the magnitude of the vector sum of the transverse momenta of all objects associated with the PP system in the lab frame ( $\left.p_{\mathrm{TPP}}^{\mathrm{lab}}\right)$ to the overall transverse scale variable considered. This quantity tests for significant boost in the transverse direction. For signal events this quantity peaks sharply towards zero while for background processes the distribution is broader. A test of how much a given process resembles the imposed PP system in the decay tree.

(iv) $H_{\mathrm{T} 3,1}^{\mathrm{PP}} / H_{3,1}^{\mathrm{PP}}$ : a measure of the fraction of the momentum that lies in the transverse plane. (v) $\min \left(H_{1,1}^{\mathrm{P}_{\mathrm{a}}}, H_{1,1}^{\mathrm{P}_{\mathrm{b}}}\right) / \min \left(H_{2,1}^{\mathrm{P}_{\mathrm{a}}}, H_{2,1}^{\mathrm{P}_{\mathrm{b}}}\right)$ : compares the scale due to one visible object and $E_{\mathrm{T}}^{\text {miss }}\left(H_{1,1}^{\mathrm{P}_{\mathrm{a}}}\right.$ and $H_{1,1}^{\mathrm{P}_{\mathrm{b}}}$ in their respective production frames) as opposed to two visible objects $\left(H_{2,1}^{\mathrm{P}_{\mathrm{a}}}\right.$ and $\left.H_{2,1}^{\mathrm{P}_{\mathrm{b}}}\right)$. The numerator and denominator are each defined by finding the minimum value of these quantities. In the three-lepton case this corresponds to the hemisphere with the $Z$ boson as it is the only one with two visible objects, and the variable takes the form $H_{1,1}^{\mathrm{P}_{\mathrm{b}}} / H_{2,1}^{\mathrm{P}_{\mathrm{b}}}$. This variable tests against a single object taking a large portion of the hemisphere momentum. This is particularly useful in discriminating against $Z+$ jets backgrounds.

(vi) $\Delta \phi_{\mathrm{V}}^{\mathrm{P}}$ : the azimuthal opening angle between the visible system $\mathrm{V}$ in frame $\mathrm{P}$ and the direction of the boost from the PP to P frame. Standard Model backgrounds from diboson, top and $Z+$ jets processes peak towards zero and $\pi$ due to their topologies not obeying the imposed decay tree while signals tend to have a flat distribution in this variable.

In addition to trying to resolve the entirety of the signal event, it can be useful for sparticle spectra with smaller mass splittings and lower intrinsic $E_{\mathrm{T}}^{\text {miss }}$ to instead select events with a partially resolved sparticle system recoiling from a high- $p_{\mathrm{T}}$ jet from ISR. To target such topologies, a separate decay tree for compressed spectra is shown in Fig. 2(d). This tree is somewhat simpler and attempts to identify visible (V) and invisible (I) systems that are the result of an intermediate state corresponding to the system of sparticles and their decay products (S). As the $E_{\mathrm{T}}^{\text {miss }}$ is used to choose which jets are identified as ISR, a transverse view of the reconstructed event is used which ignores the longitudinal momentum of the jets and leptons, as described in Ref [13]. The reference frames appearing in the decay tree shown in Fig. 2(d), such as the center-ofmass $(\mathrm{CM})$ frame of the whole reaction, are then approximations in this transverse projection. This tree yields a slightly different set of variables:

(i) $p_{\mathrm{T} \text { ISR }}^{\mathrm{CM}}$ : the magnitude of the vector-summed transverse momenta of all jets assigned to the ISR system.

(ii) $p_{\mathrm{TI}}^{\mathrm{CM}}$ : the magnitude of the vector-summed transverse momenta of the invisible system. Behaves similarly to $E_{\mathrm{T}}^{\text {miss }}$.

(iii) $p_{\mathrm{T}}^{\mathrm{CM}}$ : the magnitude of the vector-summed transverse momenta of the CM system.

(iv) $R_{\mathrm{ISR}} \equiv \vec{p}_{\mathrm{I}}^{\mathrm{CM}} \cdot \hat{p}_{\mathrm{TS}}^{\mathrm{CM}} / p_{\mathrm{TS}}^{\mathrm{CM}}$ : serves as an estimate of $m_{\tilde{\chi}_{1}^{0}} / m_{\tilde{\chi}_{2}^{0} / \tilde{\chi}_{1}^{ \pm}}$. This corresponds to the fraction of the momentum of the $\mathrm{S}$ system that is carried by its invisible system I, with momentum $\vec{p}_{\mathrm{I}}^{\mathrm{CM}}$ in the $\mathrm{CM}$ frame. As $p_{\mathrm{TS}}^{\mathrm{CM}}$ grows it becomes increasingly hard for backgrounds to possess a large value in this ratio-a feature exhibited by compressed signals [13].

(v) $N_{\text {jet }}^{\mathrm{S}}$ : number of jets assigned to the signal system $(\mathrm{S})$. 


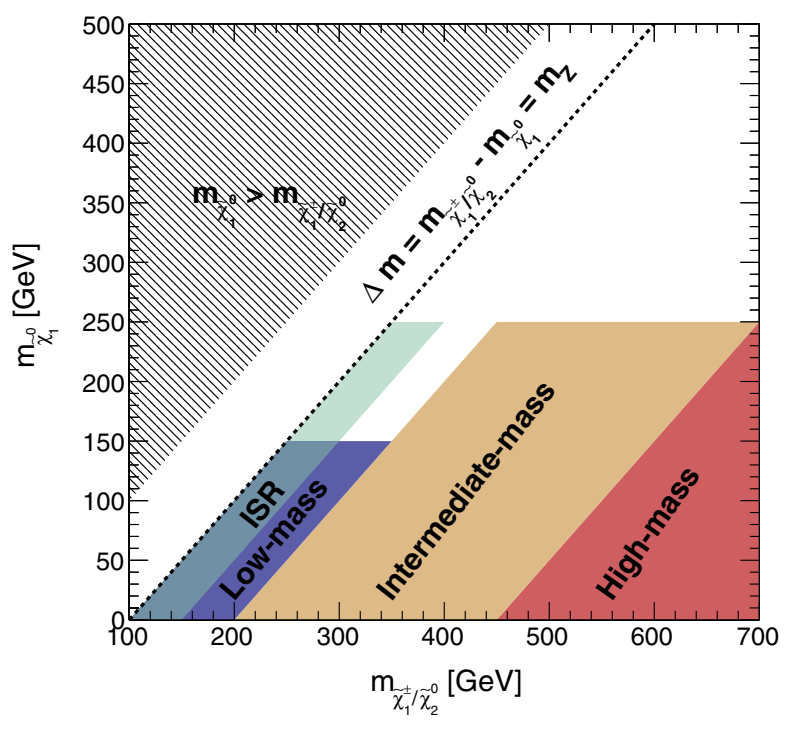

FIG. 3. Sketch of the regions that are probed by each signal region in the two-dimensional parameter space $m_{\tilde{\chi}_{1}^{ \pm} / \tilde{\chi}_{2}^{0}}-m_{\tilde{\chi}_{1}^{0}}$.

(vi) $N_{\text {jet }}^{\mathrm{ISR}}$ : number of jets assigned to the ISR system.

(vii) $\Delta \phi_{\text {ISR.I }}^{\mathrm{CM}}$ : the azimuthal opening angle between the ISR system and the invisible system in the $\mathrm{CM}$ frame.

(viii) $m_{Z}$ : mass of the dilepton pair assigned to the signal system. In the 3-lepton final state, the $Z$ candidate is formed by finding the same-flavor opposite-charge pair closest to the $Z$ mass.

(ix) $m_{J}$ : mass of the jet system assigned to the signal system.

\section{EVENT SELECTION: CONTROL, VALIDATION AND SIGNAL REGION DEFINITIONS}

Following the object reconstruction described in Sec. IV and analysis strategy outlined in Sec. V, the variables described in Sec. VI are used to define a set of SRs sensitive to the topologies of interest.

Both the $2 \ell$ and $3 \ell$ SRs are designed to cover a wide range of $\tilde{\chi}_{1}^{ \pm} / \tilde{\chi}_{2}^{0}$ masses and different mass splittings, $\Delta m=m_{\tilde{\chi}_{1}^{ \pm} / \tilde{\chi}_{2}^{0}}-m_{\tilde{\chi}_{1}^{0}}$. Specifically, the high-mass regions target high $\tilde{\chi}_{1}^{ \pm} / \tilde{\chi}_{2}^{0}$ masses and large mass splittings $(\Delta m \gtrsim 400 \mathrm{GeV})$ and the intermediate-mass regions probe mass splittings of $\approx 200 \mathrm{GeV}$. The low-mass and ISR SRs are constructed in order to probe similar regions of the twodimensional SUSY parameter space and particularly the mass splittings of $\approx 100 \mathrm{GeV}$. In this region it is difficult to distinguish the signal from SM processes, due to the limited momentum that the LSPs carry. Improved sensitivity is achieved by designing the two low-mass and ISR SRs to be mutually exclusive, with each providing sensitivity to the parameter space under scrutiny. A statistical combination of these regions subsequently leads to further improved sensitivities. A schematic representation of the mass regions targeted by each SR can be seen in Fig. 3 .

For selections involving three charged leptons, the $W$ boson transverse mass, $m_{\mathrm{T}}^{W}$, is used and is derived from $\vec{p}_{\mathrm{T}}^{\text {miss }}$ and the transverse momentum of the charged lepton $\left(p_{\mathrm{T}}^{\ell}\right)$ not associated with the $Z$ boson as follows:

$$
m_{\mathrm{T}}^{W}=\sqrt{2 p_{\mathrm{T}}^{\ell} E_{\mathrm{T}}^{\mathrm{miss}}(1-\cos \Delta \phi)},
$$

where $\Delta \phi$ is the azimuthal opening angle between the charged lepton associated with the $W$ boson and the missing transverse momentum.

\section{A. Event selection in the two-lepton channel}

The $2 \ell$ search channel, using the standard decay tree, is designed with three SRs, two CRs to constrain the $V V$ background (where $V=W, Z$ ) and the processes with top quarks $(W t+t \bar{t}$, where the sign symbolizes the sum of the two processes) and four VRs for validating the main

TABLE II. Preselection criteria for the three standard-decay-tree $2 \ell$ SRs and the associated CRs and VRs. The variables are defined in the text.

\begin{tabular}{|c|c|c|c|c|c|c|c|c|}
\hline Region & $n_{\text {leptons }}$ & $n_{\text {jets }}$ & $n_{b-\operatorname{tag}}$ & $p_{\mathrm{T}}^{\ell_{1}, \ell_{2}}[\mathrm{GeV}]$ & $p_{\mathrm{T}}^{j_{1}, j_{2}}[\mathrm{GeV}]$ & $m_{\ell \ell}[\mathrm{GeV}]$ & $m_{j j}[\mathrm{GeV}]$ & $m_{\mathrm{T}}^{W}[\mathrm{GeV}]$ \\
\hline $\mathrm{CR} 2 \ell-\mathrm{VV}$ & $\in[3,4]$ & $\geq 2$ & $=0$ & $>25$ & $>30$ & $\in(80,100)$ & $>20$ & $\in(70,100)$ if $n_{\text {leptons }}=3$ \\
\hline CR2 $\ell$-Top & $=2$ & $\geq 2$ & $=1$ & $>25$ & $>30$ & $\in(80,100)$ & $\in(40,250)$ & $\cdots$ \\
\hline $\mathrm{VR} 2 \ell-\mathrm{VV}$ & $=2$ & $\geq 2$ & $=0$ & $>25$ & $>30$ & $\in(80,100)$ & $\begin{array}{c}\in(40,70) \\
\text { or } \in(90,500)\end{array}$ & $\begin{array}{l}\cdots \\
\cdots\end{array}$ \\
\hline VR2 $\ell$-Top & $=2$ & $\geq 2$ & $=1$ & $>25$ & $>30$ & $\begin{array}{c}\in(20,80) \\
\text { or }>100\end{array}$ & $\in(40,250)$ & $\begin{array}{l}\cdots \\
\cdots\end{array}$ \\
\hline VR2 $\ell$ High-Zjets & $=2$ & $\geq 2$ & $=0$ & $>25$ & $>30$ & $\in(80,100)$ & $\begin{array}{c}\in(0,60) \\
\text { or } \in(100,180)\end{array}$ & $\begin{array}{l}\cdots \\
\cdots\end{array}$ \\
\hline VR2 $\ell$ Low-Zjets & $=2$ & $=2$ & $=0$ & $>25$ & $>30$ & $\in(80,100)$ & $\begin{array}{c}\in(0,60) \\
\text { or } \in(100,180)\end{array}$ & $\begin{array}{l}\cdots \\
\cdots\end{array}$ \\
\hline SR $2 \ell$ High & $=2$ & $\geq 2$ & $=0$ & $>25$ & $>30$ & $\in(80,100)$ & $\in(60,100)$ & $\cdots$ \\
\hline SR $2 \ell$ Int & $=2$ & $\geq 2$ & $=0$ & $>25$ & $>30$ & $\in(80,100)$ & $\in(60,100)$ & $\ldots$ \\
\hline SR2 $\ell$ _Low & $=2$ & $=2$ & $=0$ & $>25$ & $>30$ & $\in(80,100)$ & $\in(70,90)$ & . \\
\hline
\end{tabular}


TABLE III. Selection criteria for the three standard-decay-tree $2 \ell$ SRs and the associated CRs and VRs. The variables are defined in the text.

\begin{tabular}{|c|c|c|c|c|c|c|c|}
\hline Region & $H_{4,1}^{\mathrm{PP}}[\mathrm{GeV}]$ & $H_{1,1}^{\mathrm{PP}}[\mathrm{GeV}]$ & $\frac{p_{\mathrm{TPP}}^{\mathrm{lab}}}{p_{\mathrm{TPP}}^{\mathrm{aab}}+H_{\mathrm{T} 4,1}^{\mathrm{PP}}}$ & $\frac{\min \left(H_{1,1}^{\mathrm{P}_{\mathrm{a}}}, H_{1,1}^{\mathrm{P}_{\mathrm{b}}}\right)}{\min \left(H_{2,1}^{\mathrm{Pa}_{2}}, H_{2,1}^{\mathrm{P}_{\mathrm{b}}}\right)}$ & $\frac{H_{1,1}^{\mathrm{PP}}}{H_{4,1}^{\mathrm{PP}}}$ & $\Delta \phi_{\mathrm{V}}^{\mathrm{P}}$ & $\min \Delta \phi\left(j_{1} / j_{2}, \vec{p}_{\mathrm{T}}^{\text {miss }}\right)$ \\
\hline $\mathrm{CR} 2 \ell-\mathrm{VV}$ & $>200$ & $\cdots$ & $<0.05$ & $>0.2$ & $\ldots$ & $\in(0.3,2.8)$ & $\ldots$ \\
\hline CR2 $\ell$-Top & $>400$ & $\cdots$ & $<0.05$ & $>0.5$ & $\cdots$ & $\in(0.3,2.8)$ & $\cdots$ \\
\hline $\mathrm{VR} 2 \ell-\mathrm{VV}$ & $>400$ & $>250$ & $<0.05$ & $\in(0.4,0.8)$ & $\ldots$ & $\in(0.3,2.8)$ & $\ldots$ \\
\hline VR2 $\ell$-Top & $>400$ & $\cdots$ & $<0.05$ & $>0.5$ & $\cdots$ & $\in(0.3,2.8)$ & $\cdots$ \\
\hline VR2 $\ell$ High-Zjets & $>600$ & $\cdots$ & $<0.05$ & $>0.4$ & $\cdots$ & $\in(0.3,2.8)$ & $\cdots$ \\
\hline VR2 $\ell$ _Low-Zjets & $>400$ & $\cdots$ & $<0.05$ & $\cdots$ & $\in(0.35,0.60)$ & $\cdots$ & $\cdots$ \\
\hline SR2 $\ell$ _High & $>800$ & $\cdots$ & $<0.05$ & $>0.8$ & $\cdots$ & $\in(0.3,2.8)$ & $\ldots$ \\
\hline $\mathrm{SR} 2 \ell_{\text {_Int }}$ & $>600$ & $\cdots$ & $<0.05$ & $>0.8$ & $\cdots$ & $\in(0.6,2.6)$ & $\cdots$ \\
\hline SR2 $\ell_{\text {_L Low }}$ & $>400$ & $\cdots$ & $<0.05$ & $\cdots$ & $\in(0.35,0.60)$ & $\cdots$ & $>2.4$ \\
\hline
\end{tabular}

TABLE IV. Preselection criteria for the compressed-decay-tree $2 \ell \mathrm{SR}$ and the associated CRs and VRs. The variables are defined in the text.

\begin{tabular}{lccccccc}
\hline \hline Region & $n_{\text {leptons }}$ & $N_{\text {jet }}^{\text {ISR }}$ & $N_{\text {jet }}^{\mathrm{S}}$ & $n_{\text {jets }}$ & $n_{b \text {-tag }}$ & $p_{\mathrm{T}}^{\ell_{1}, \ell_{2}}[\mathrm{GeV}]$ & $p_{\mathrm{T}}^{j_{1}, j_{2}}[\mathrm{GeV}]$ \\
\hline CR2 $\ell$ ISR-VV & $\in[3,4]$ & $\geq 1$ & $\geq 2$ & $>2$ & $=0$ & $>25$ & $>30$ \\
CR2 $\ell$ ISR-Top & $=2$ & $\geq 1$ & $=2$ & $\in[3,4]$ & $=1$ & $>25$ & $>30$ \\
VR2 $\ell$ ISR-VV & $\in[3,4]$ & $\geq 1$ & $\geq 2$ & $\geq 3$ & $=0$ & $>25$ & $>20$ \\
VR2 $\ell$ ISR-Top & $=2$ & $\geq 1$ & $=2$ & $\in[3,4]$ & $=1$ & $>25$ & $>30$ \\
VR2 $\ell$ _ISR-Zjets & $=2$ & $\geq 1$ & $\geq 1$ & $\in[3,5]$ & $=0$ & $>25$ & $>30$ \\
SR2 $\ell$ IISR & $=2$ & $\geq 1$ & $=2$ & $\in[3,4]$ & $=0$ & $>25$ & $>30$ \\
\hline \hline
\end{tabular}

background processes (including the $Z+$ jets data-driven estimate described in Sec. VIII A). The preselection criteria used for the definition of the standard-decay-tree regions are listed in Table II and include requirements on the lepton multiplicity ( $\left.n_{\text {leptons }}\right)$, the jet multiplicity $\left(n_{\text {jets }}\right)$, the $b$-tag jet multiplicity $\left(n_{b \text {-tag }}\right)$, the transverse momenta of the leading $\left(p_{\mathrm{T}}^{\ell_{1}}, p_{\mathrm{T}}^{j_{1}}\right)$ and subleading $\left(p_{\mathrm{T}}^{\ell_{2}}, p_{\mathrm{T}}^{j_{2}}\right)$ leptons and jets and the invariant mass of the dilepton $\left(m_{\ell \ell}\right)$ and dijet $\left(m_{j j}\right)$ system. Most of the regions are defined with exactly two oppositecharge, same-flavor leptons with transverse momentum greater than $25 \mathrm{GeV}$ and an invariant mass consistent with arising from a $Z$ boson. Exceptions to this are the diboson $\mathrm{CR}(\mathrm{CR} 2 \ell-\mathrm{VV})$ and top VR (VR2 $\ell$-Top). The CR2 $\ell$-VV requires three or four leptons, which helps to select a sample enriched in diboson events as well as to ensure orthogonality with the SRs. The lepton pair is selected by choosing the opposite-charge, same-flavor pair closest to the $Z$ mass, while the remaining lepton(s) are treated as invisible objects contributing to $\vec{p}_{\mathrm{T}}^{\text {miss }}$. The additional requirement on $m_{\mathrm{T}}^{W}$, which is applied only in the events containing exactly three charged leptons, ensures orthogonality with the $3 \ell$ regions described in Sec. VII B. Both the top CR (CR2 $\ell$-Top) and VR (VR2 $\ell$-Top) are defined with a $b$-tag jet requirement while orthogonality with each other is ensured by inverting the dilepton invariant mass requirement. In all regions the dijet invariant mass is

TABLE V. Selection criteria for the compressed-decay-tree $2 \ell \mathrm{SR}$ and the associated CRs and VRs. The variables are defined in the text.

\begin{tabular}{lccccccc}
\hline \hline Region & $m_{Z}[\mathrm{GeV}]$ & $m_{J}[\mathrm{GeV}]$ & $\Delta \phi_{\mathrm{ISR}, \mathrm{I}}^{\mathrm{CM}}$ & $R_{\mathrm{ISR}}$ & $p_{\mathrm{TISR}}^{\mathrm{CM}}[\mathrm{GeV}]$ & $p_{\mathrm{TI}}^{\mathrm{CM}}[\mathrm{GeV}]$ & $p_{\mathrm{T}}^{\mathrm{CM}}[\mathrm{GeV}]$ \\
\hline CR2 $\ell$ _ISR-VV & $\in(80,100)$ & $>20$ & $>2.0$ & $\in(0.0,0.5)$ & $>50$ & $>50$ & $<30$ \\
CR2 $\ell$ IISR-Top & $\in(50,200)$ & $\in(50,200)$ & $>2.8$ & $\in(0.4,0.75)$ & $>180$ & $>100$ & $<20$ \\
VR2 $\ell$ IISR-VV & $\in(20,80)$ & $>20$ & $>2.0$ & $\in(0.0,1.0)$ & $>70$ & $>70$ & $<30$ \\
& or $>100$ & & & & & $>100$ & $>20$ \\
VR2 $\ell$ _ISR-Top & $\in(50,200)$ & $\in(50,200)$ & $>2.8$ & $\in(0.4,0.75)$ & $>180$ & $>100$ & $<20$ \\
VR2 $\ell$ ISR-Zjets & $\in(80,100)$ & $<50$ or $>110$ & $\cdots$ & $\cdots$ & $>180$ & $>100$ & $>20$ \\
SR2 $\ell$ _ISR & $\in(80,100)$ & $\in(50,110)$ & $>2.8$ & $\in(0.4,0.75)$ & $>180$ & $>100$ & $<20$ \\
\hline \hline
\end{tabular}



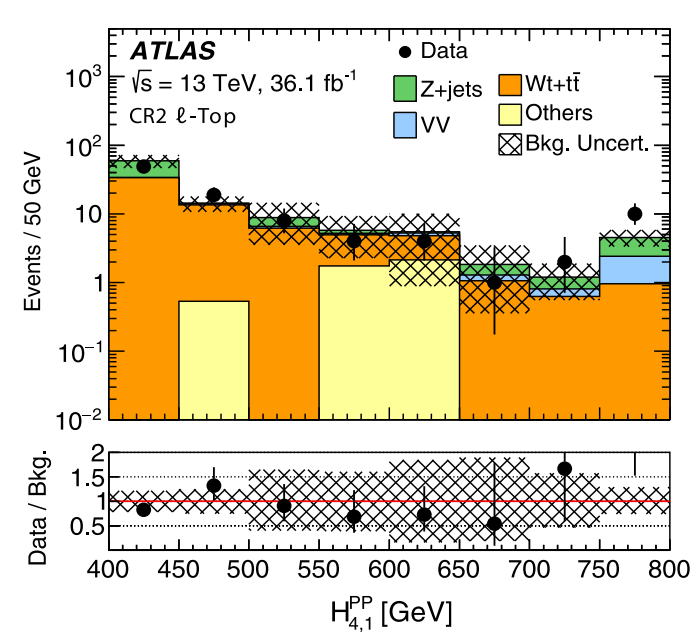

(a)
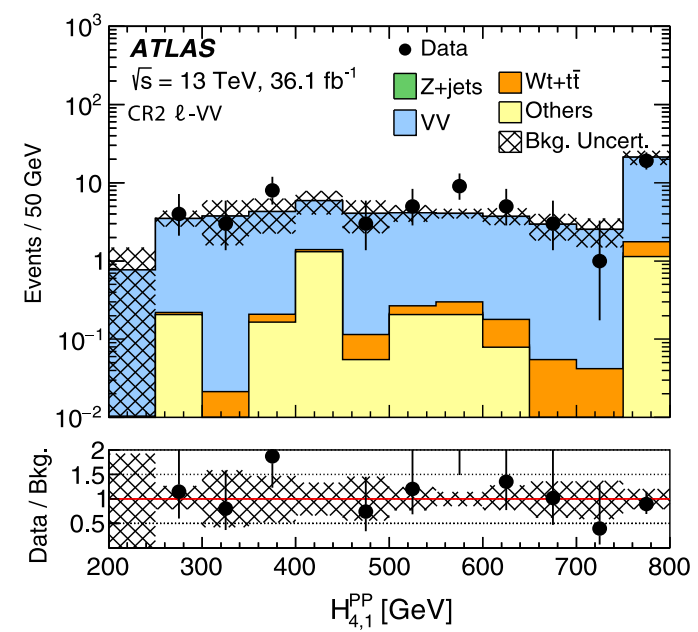

(c)
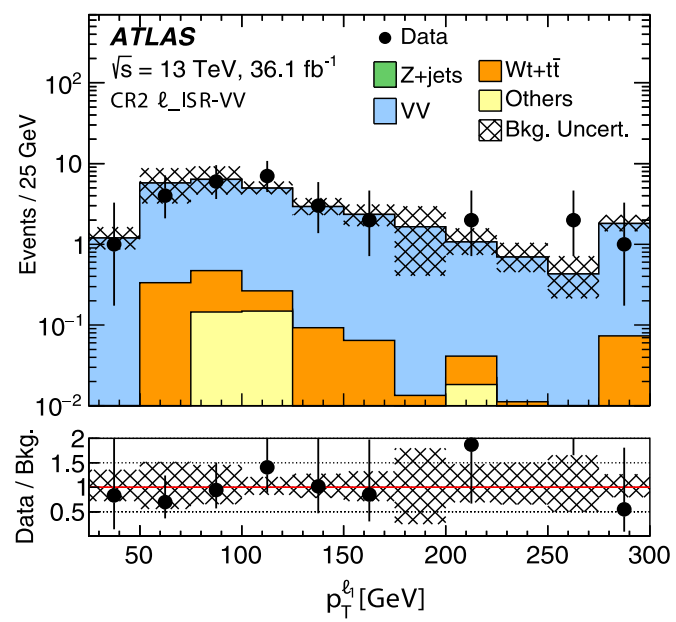

(e)
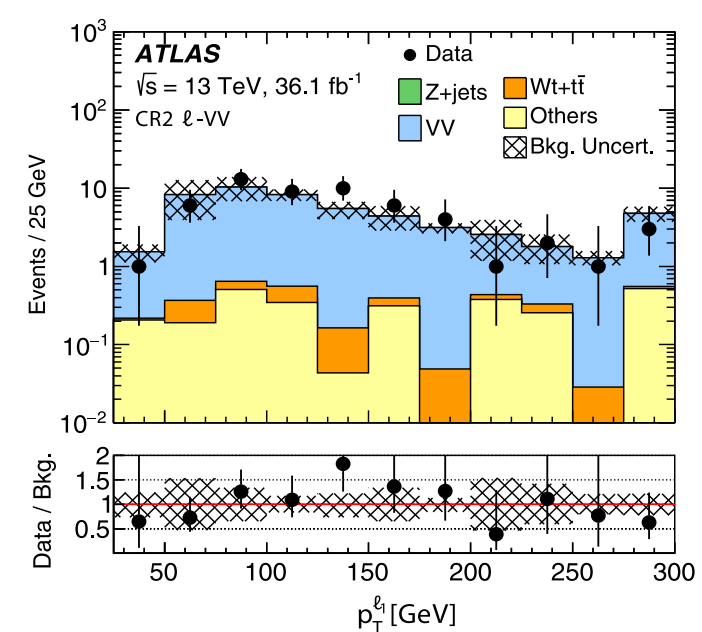

(b)
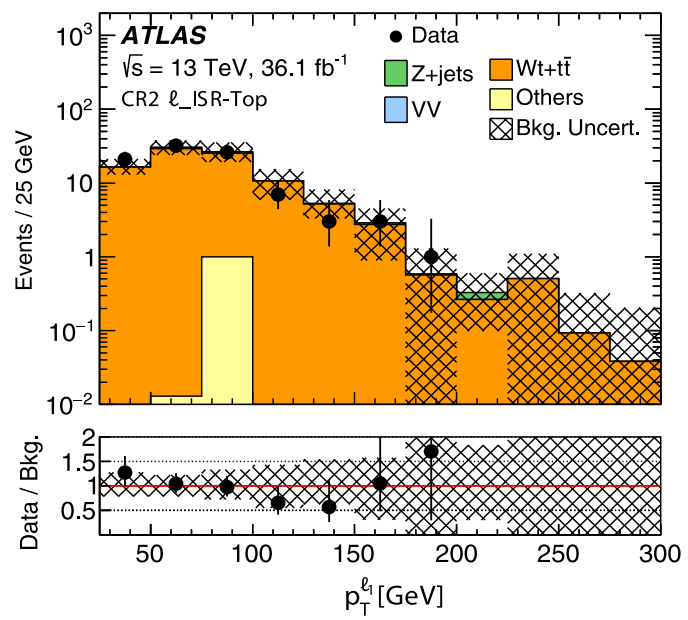

(d)

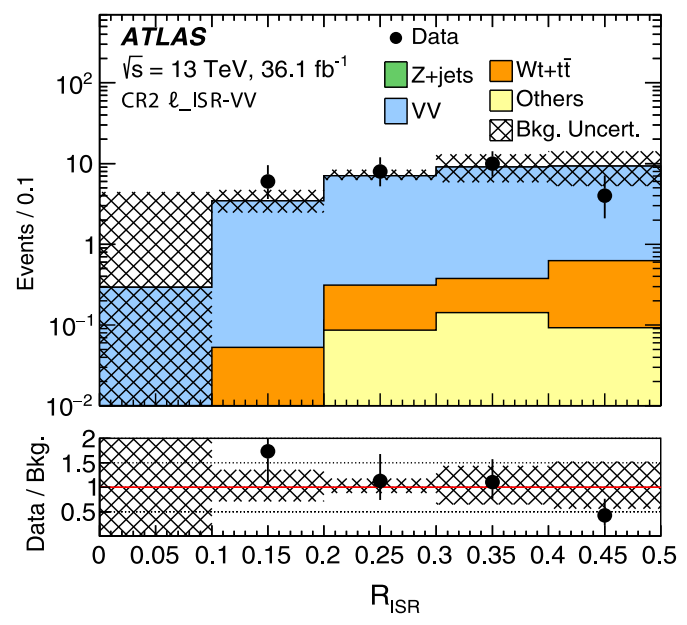

(f)

FIG. 4. Distributions of kinematic variables in the control regions for the $2 \ell$ channel after applying all selection requirements in Tables III or V. The histograms show the postfit MC background predictions. The last bin includes the overflow. The FNP contribution is estimated from a data-driven technique and is included in the category "Others." Distributions for the (a) $H_{4,1}^{\mathrm{PP}}$ standard-decay-tree top $\mathrm{CR}$, (b) $p_{\mathrm{T}}^{\ell_{1}}$ and (c) $H_{4,1}^{\mathrm{PP}}$ for the standard decay tree VV CR, (d) $p_{\mathrm{T}}^{\ell_{1}}$ compressed-decay-tree top CR, and (e) $p_{\mathrm{T}}^{\ell_{1}}$ compressed-decay-tree $V V$ CR and (f) $R_{\text {ISR }}$ compressed-decay-tree $V V$ CR are plotted. The hatched error bands indicate the combined theoretical, experimental and MC statistical uncertainties. 


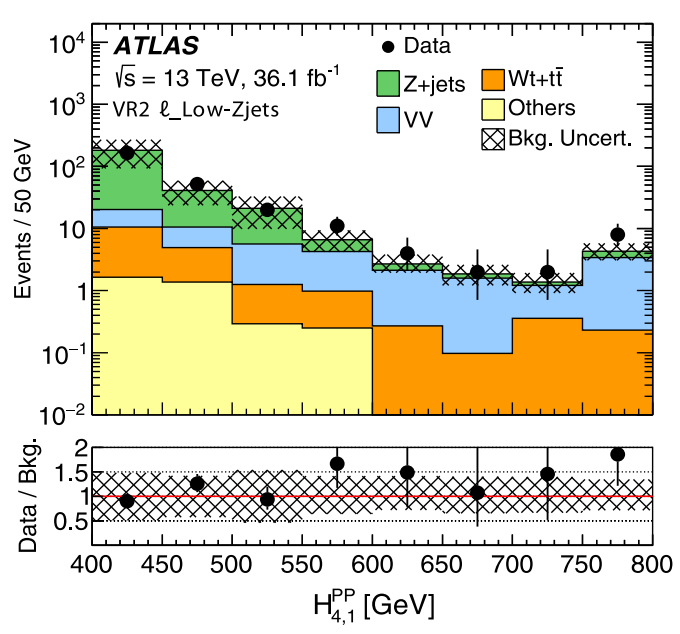

(a)

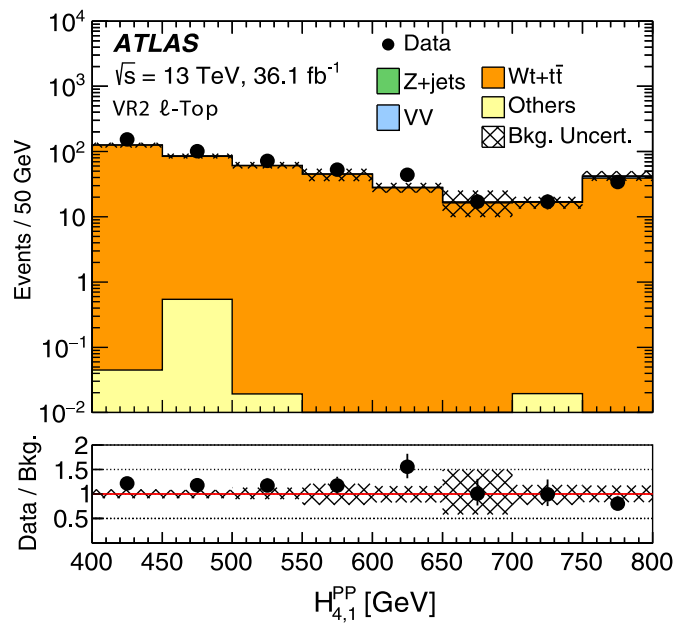

(c)

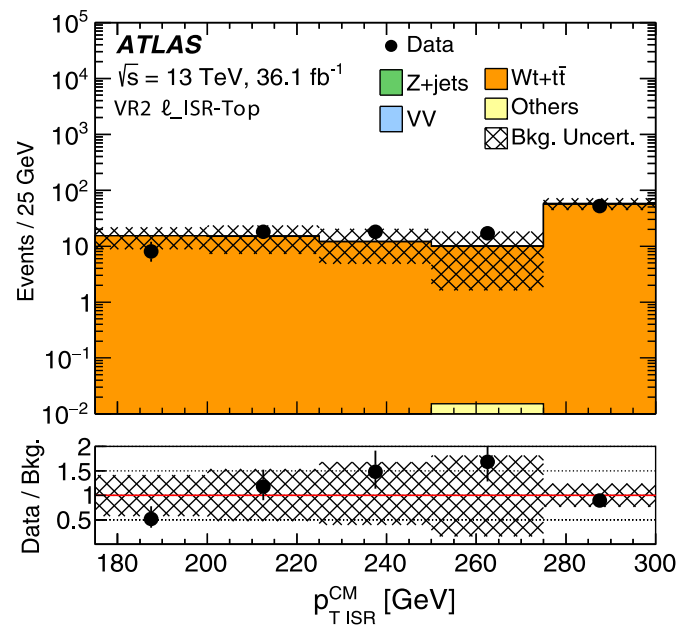

(e)
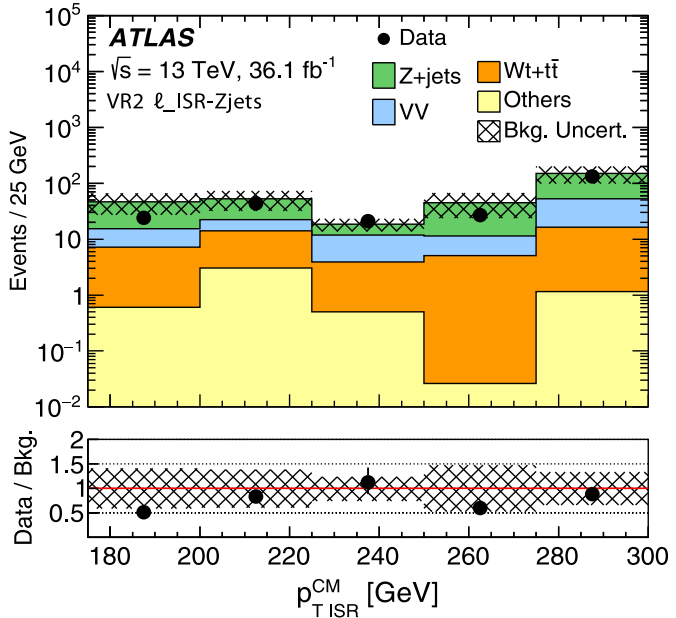

(b)

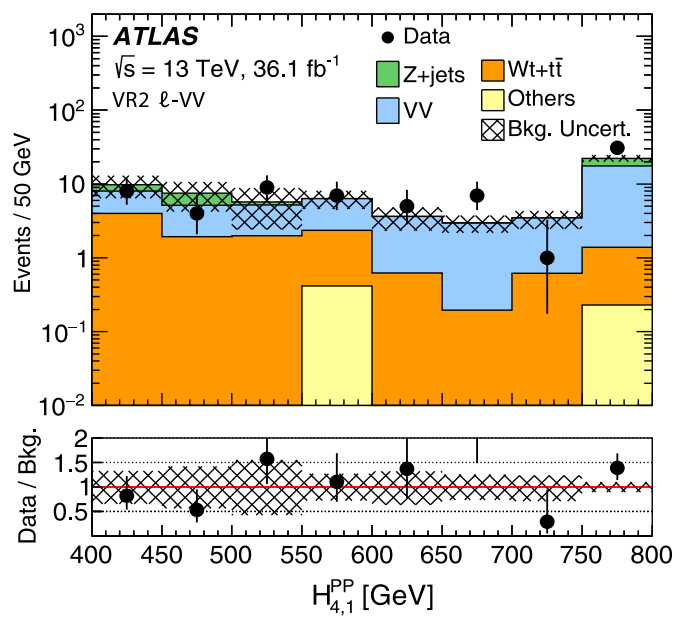

(d)

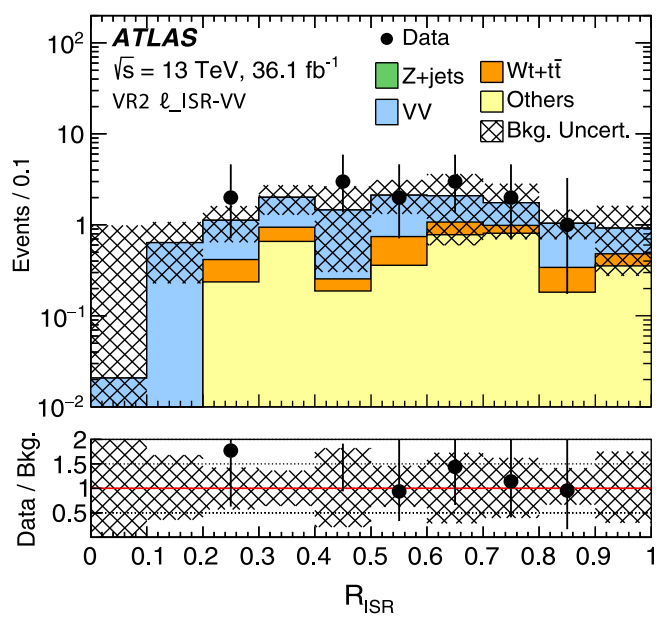

(f)

FIG. 5. Distributions of kinematic variables in the validation regions for the $2 \ell$ channel after applying all selection requirements in Tables III or V. The histograms show the postfit MC background predictions. The last bin includes the overflow. The FNP contribution is estimated from a data-driven technique and is included in the category "Others." Plots show (a) $H_{4,1}^{\mathrm{PP}}$ and (b) $p_{\mathrm{TISR}}^{\mathrm{CM}}$ in the $Z+$ jets VRs for the standard and compressed decay trees respectively; (c) $H_{4,1}^{\mathrm{PP}}$ in the top VR and (d) $H_{4,1}^{\mathrm{PP}}$ in the diboson VR for the standard decay tree; (e) $p_{\mathrm{TISR}}^{\mathrm{CM}}$ in the top VR and (f) $R_{\mathrm{ISR}}$ in the diboson VR for the compressed decay tree. The hatched error bands indicate the combined theoretical, experimental and MC statistical uncertainties. 
TABLE VI. Preselection criteria for the $3 \ell \mathrm{CR}, \mathrm{VR}$ and $\mathrm{SR}$ with the standard decay tree. The variables are defined in the text.

\begin{tabular}{|c|c|c|c|c|c|c|}
\hline Region & $n_{\text {leptons }}$ & $n_{\text {jets }}$ & $n_{b-\operatorname{tag}}$ & $p_{\mathrm{T}}^{\ell_{1}}[\mathrm{GeV}]$ & $p_{\mathrm{T}}^{\ell_{2}}[\mathrm{GeV}]$ & $p_{\mathrm{T}}^{\ell_{3}}[\mathrm{GeV}]$ \\
\hline $\mathrm{CR} 3 \ell-\mathrm{VV}$ & $=3$ & $<3$ & $=0$ & $>60$ & $>40$ & $>30$ \\
\hline $\mathrm{VR} 3 \ell-\mathrm{VV}$ & $=3$ & $<3$ & $=0$ & $>60$ & $>40$ & $>30$ \\
\hline SR3 $\ell$ _High & $=3$ & $<3$ & $=0$ & $>60$ & $>60$ & $>40$ \\
\hline $\mathrm{SR} 3 \ell$ Int & $=3$ & $<3$ & $=0$ & $>60$ & $>50$ & $>30$ \\
\hline SR3 $\ell \_$Low & $=3$ & $=0$ & $=0$ & $>60$ & $>40$ & $>30$ \\
\hline
\end{tabular}

TABLE VII. Selection criteria for the $3 \ell$ CR VR and SR with the standard decay tree. The variables are defined in the text.

\begin{tabular}{|c|c|c|c|c|c|c|}
\hline Region & $m_{\ell \ell}[\mathrm{GeV}]$ & $m_{\mathrm{T}}^{W}[\mathrm{GeV}]$ & $H_{3,1}^{\mathrm{PP}}[\mathrm{GeV}]$ & $\frac{p_{\mathrm{TPP}}^{\mathrm{lab}}}{p_{\mathrm{TPP}}^{\mathrm{lab}}+H_{\mathrm{T} 3,1}^{\mathrm{PP}}}$ & $\frac{H_{\mathrm{TP}, 1}^{\mathrm{PP}}}{H_{3,1}^{\mathrm{PP}}}$ & $\frac{H_{1,1}^{\mathrm{Pb}_{\mathrm{b}}}}{H_{2,1}^{\mathrm{P}_{\mathrm{b}}}}$ \\
\hline $\mathrm{CR} 3 \ell-\mathrm{VV}$ & $\in(75,105)$ & $\in(0,70)$ & $>250$ & $<0.2$ & $>0.75$ & $\ldots$ \\
\hline $\mathrm{VR} 3 \ell-\mathrm{VV}$ & $\in(75,105)$ & $\in(70,100)$ & $>250$ & $<0.2$ & $>0.75$ & $\ldots$ \\
\hline SR3 $\ell$ _High & $\in(75,105)$ & $>150$ & $>550$ & $<0.2$ & $>0.75$ & $>0.8$ \\
\hline SR3 $\ell$ Int & $\in(75,105)$ & $>130$ & $>450$ & $<0.15$ & $>0.8$ & $>0.75$ \\
\hline SR3 $\ell$ Low & $\in(75,105)$ & $>100$ & $>250$ & $<0.05$ & $>0.9$ & $\ldots$ \\
\hline
\end{tabular}

TABLE VIII. Preselection criteria for the $3 \ell \mathrm{CR}$, VR and SR with the compressed decay tree. The variables are defined in the text.

\begin{tabular}{|c|c|c|c|c|c|c|}
\hline Region & $n_{\text {leptons }}$ & $n_{\text {jets }}$ & $n_{b \text {-tag }}$ & $p_{\mathrm{T}}^{\ell_{1}}[\mathrm{GeV}]$ & $p_{\mathrm{T}}^{\ell_{2}}[\mathrm{GeV}]$ & $p_{\mathrm{T}}^{\ell_{3}}[\mathrm{GeV}]$ \\
\hline CR3 3 _ISR-VV & $=3$ & $\geq 1$ & $=0$ & $>25$ & $>25$ & $>20$ \\
\hline VR3 $\ell$ ISR-VV & $=3$ & $\geq 1$ & $=0$ & $>25$ & $>25$ & $>20$ \\
\hline $\mathrm{SR} 3 \ell$ ISR & $=3$ & $\in[1,3]$ & $=0$ & $>25$ & $>25$ & $>20$ \\
\hline
\end{tabular}

formed using the two leading jets in $p_{\mathrm{T}}$. The SRs require the $m_{j j}$ to be consistent with a $W$ boson while the $Z+$ jets (VR2 $\ell$ _High-Zjets and VR2 $\ell$ _Low-Zjets) and diboson (VR2 $\ell$-VV) VRs select events outside of the $W$ mass window.

In addition to the preselection criteria, further selection requirements are applied in each region according to the parameter space probed. These selection requirements are shown in Table III. The $\min \Delta \phi\left(j_{1} / j_{2}, \vec{p}_{\mathrm{T}}^{\text {miss }}\right)$ variable corresponds to the minimum azimuthal angle between the jets and $\vec{p}_{\mathrm{T}}^{\text {miss }}$ and is applied only in SR $2 \ell$ _Low to further suppress the $Z+$ jets contribution. The selection criteria applied in VR $2 \ell$ _High-Zjets and VR2 $\ell$ _Low-Zjets differ so as to be closer and orthogonal to their respective
SRs. As such the $0.35<H_{1,1}^{\mathrm{PP}} / H_{4,1}^{\mathrm{PP}}<0.6$ requirement is retained only for VR $2 \ell$ LLow-Zjets. VR $2 \ell-\mathrm{VV}$ is the only region with an $H_{1,1}^{\mathrm{PP}}$ requirement, but one that is necessary since it further suppresses the $Z+$ jets background while keeping the VRs close to the SRs.

Similar to the $2 \ell$ standard-decay-tree regions, another set of $2 \ell$ regions is defined by taking advantage of the compressed decay tree. SR $2 \ell$ ISR has a requirement of at least three jets which makes it orthogonal to SR2 $\ell$ Low, where the jet multiplicity is defined with exactly two jets. The lepton and jet multiplicities as well as the requirements on the transverse momenta of these objects defining the preselection requirements in the ISR analysis are summarized in Table IV. All the regions require at least one jet

TABLE IX. Selection criteria for the $3 \ell \mathrm{CR}, \mathrm{VR}$ and SR with the compressed decay tree. The variables are defined in the text.

\begin{tabular}{lccccccc}
\hline \hline Region & $m_{\ell \ell}[\mathrm{GeV}]$ & $m_{\mathrm{T}}^{W}[\mathrm{GeV}]$ & $\Delta \phi_{\mathrm{ISR}, \mathrm{I}}^{\mathrm{CM}}$ & $R_{\mathrm{ISR}}$ & $p_{\mathrm{TISR}}^{\mathrm{CM}}[\mathrm{GeV}]$ & $p_{\mathrm{TI}}^{\mathrm{CM}}[\mathrm{GeV}]$ & $p_{\mathrm{T}}^{\mathrm{CM}}[\mathrm{GeV}]$ \\
\hline CR3 $\ell$ IISR-VV & $\in(75,105)$ & $<100$ & $>2.0$ & $\in(0.55,1.0)$ & $>80$ & $>60$ & $<25$ \\
VR3 $\ell$ ISR-VV & $\in(75,105)$ & $>60$ & $>2.0$ & $\in(0.55,1.0)$ & $>80$ & $>60$ & $>25$ \\
SR3 $\ell$ IISR & $\in(75,105)$ & $>100$ & $>2.0$ & $\in(0.55,1.0)$ & $>100$ & $>80$ & $<25$ \\
\hline \hline
\end{tabular}


assigned to the ISR system $\left(N_{\text {jet }}^{\mathrm{ISR}}\right)$ and at least two jets in the signal system $\left(N_{\text {jet }}^{\mathrm{S}}\right)$ in the construction of the compressed decay tree. The assignment of the jets in the two systems results from a mass minimization performed in the $\mathrm{CM}$ frame. Following the same strategy as for the $\mathrm{CR} 2 \ell_{-} \mathrm{VV}$, both CR $2 \ell$ ISR-VV and VR $2 \ell$ ISR-VV are defined with three or four leptons. To increase the number

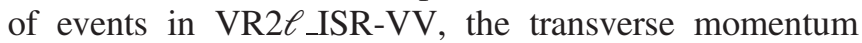
requirement for jets is relaxed to $20 \mathrm{GeV}$ compared to $30 \mathrm{GeV}$ in the other regions.

The ISR regions are further defined with a series of requirements based on the variables reconstructed from the compressed decay tree. These requirements are listed in Table V. The ISR SR is defined by requiring a highly energetic ISR jet system which recoils against the entire signal system in the CM frame. In VR2 $\ell$ ISR-VV the $m_{Z}$ requirement is inverted in order to be orthogonal to the CR2 $\ell$ _ISR-VV. The top CRs (CR2 $\ell$ _ISR-Top) and VR (VR2 $\ell$ _ISR-Top) are defined with a $b$-tag jet requirement and have broader $m_{Z}$ and $m_{J}$ windows. The broader mass windows help to increase the numbers of data events in these regions since in processes with top quarks the leptons and jets result from sources other than $Z$ and $W$ bosons, respectively. The orthogonality of the two regions is achieved by inverting the $p_{\mathrm{T}}^{\mathrm{CM}}$ requirement. A validation region for $Z+$ jets (VR2 $\ell$ _ISR-Zjets) is defined with exactly two leptons and between three and five jets, none of which are $b$-tagged; $m_{J}$ must be outside of the range expected from vector-boson decays $(<50 \mathrm{GeV}$ or $>110 \mathrm{GeV}$ ).
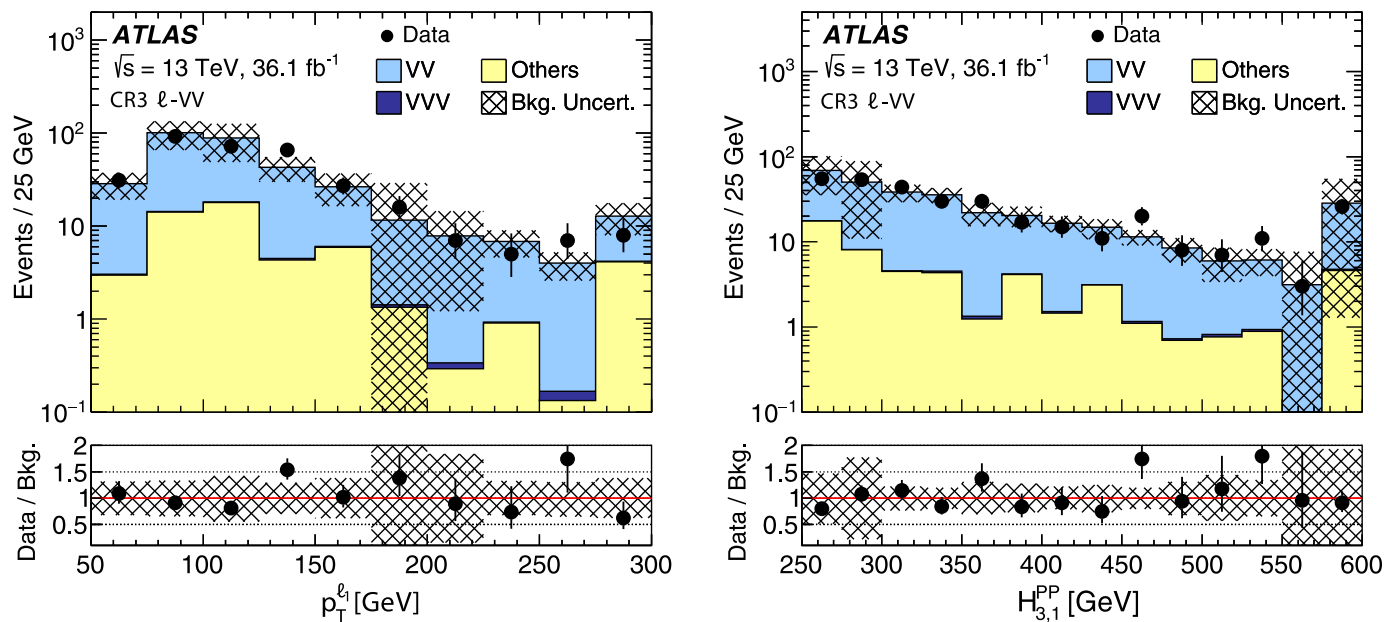

(b)

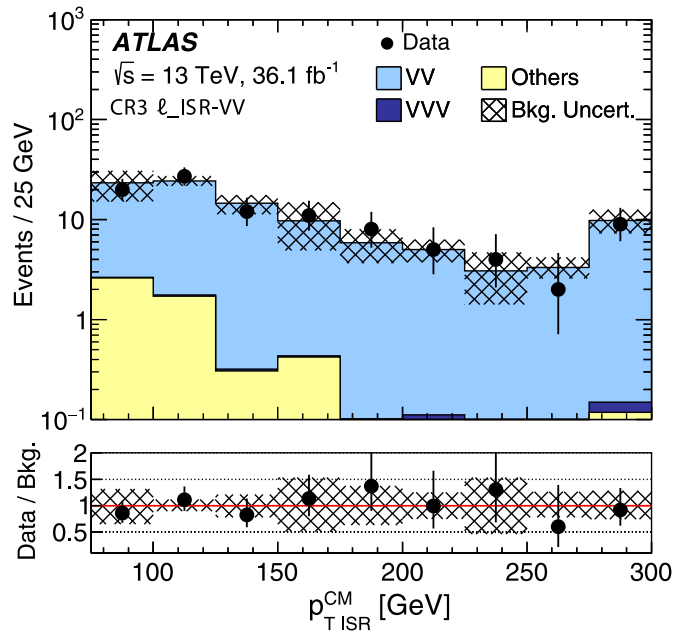

(c)

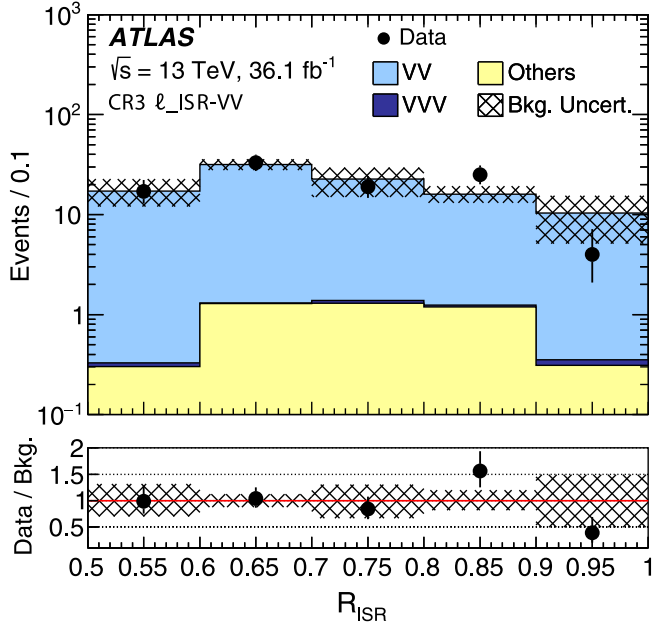

(d)

FIG. 6. Distributions of kinematic variables in the control regions for the $3 \ell$ channel after applying all selection criteria described in Tables VII or IX. The histograms show the postfit MC background predictions. The FNP contribution is estimated from a data-driven technique and is included in the category "Others." The last bin includes the overflow. Plots show (a) $p_{\mathrm{T}}^{\ell_{1}}$ and (b) $H_{3,1}^{\mathrm{PP}}$ for the diboson CR in the standard decay tree, (c) $p_{\mathrm{T} \text { ISR }}^{\mathrm{CM}}$ and (d) $R_{\mathrm{ISR}}$ for the diboson CR in the compressed decay tree. The hatched error bands indicate the combined theoretical, experimental and MC statistical uncertainties. 

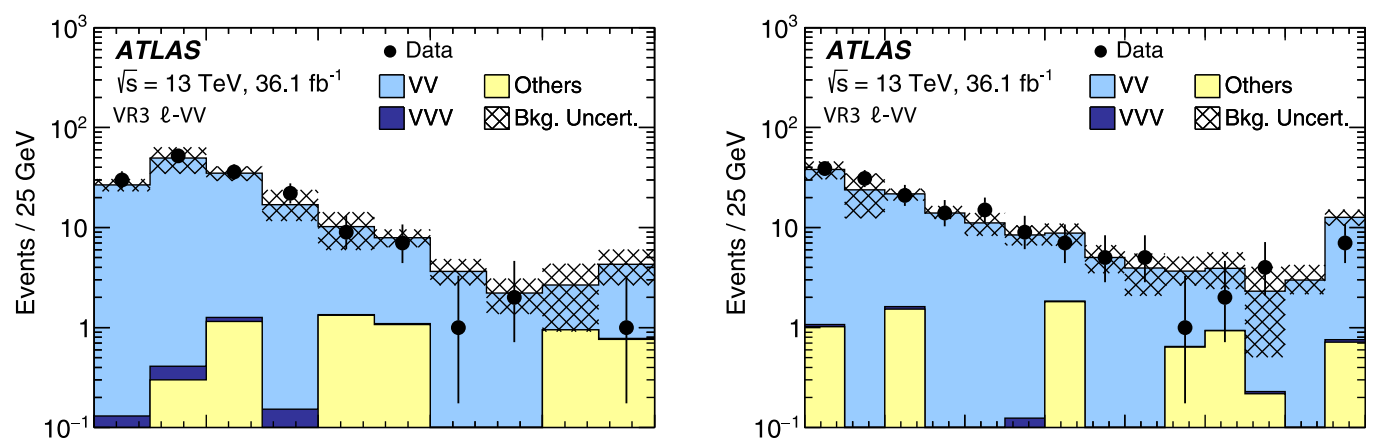

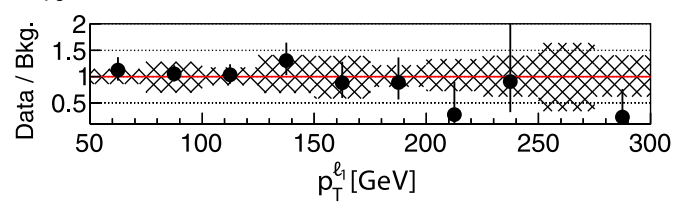

(a)

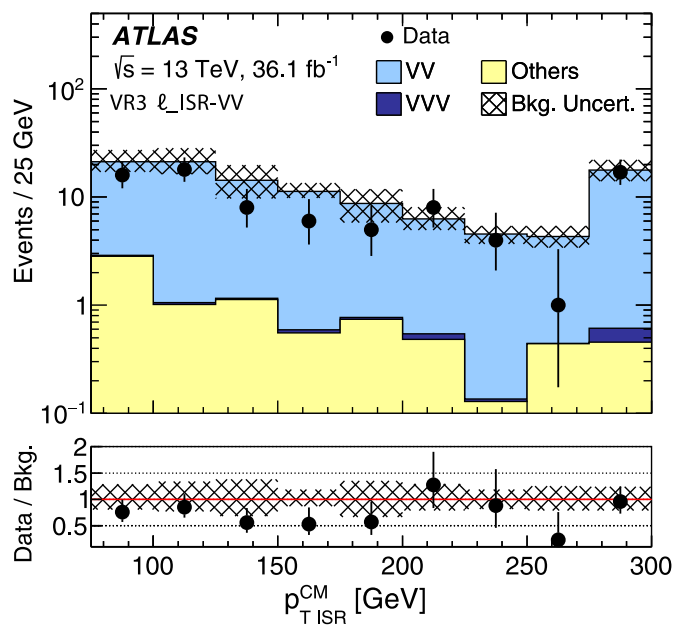

(c)

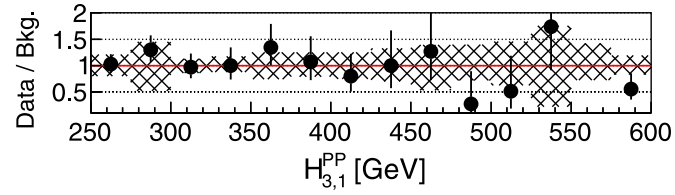

(b)

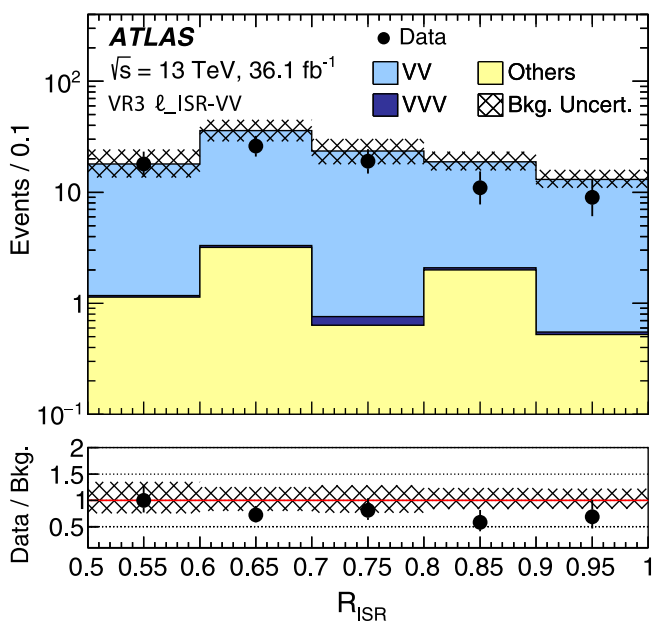

(d)

FIG. 7. Distributions of kinematic variables in the validation regions for the $3 \ell$ channel after applying all selection criteria in Tables VII or IX. The histograms show the postfit MC background predictions. The FNP contribution is estimated from a data-driven technique and is included in the category "Others." The last bin includes the overflow. Plots show (a) $p_{\mathrm{T}}^{\ell_{1}}$ and (b) $H_{3,1}^{\mathrm{PP}}$ for the standard decay tree, (c) $p_{\mathrm{TISR}}^{\mathrm{CM}}$ and (d) $R_{\mathrm{ISR}}$ for the compressed decay tree. The hatched error bands indicate the combined theoretical, experimental and MC statistical uncertainties.

Postfit distributions of variables from the $2 \ell$ search for selected regions are shown in Figs. 4 and 5 for data and the different MC samples. In these figures, the background component labeled as "Others" includes the SM contributions from Higgs boson, $V \gamma, V V V, t \bar{t} V$ production and contributions from nonprompt and nonisolated leptons. The background estimate is described in Sec. VIII.

\section{B. Event selection in the three-lepton channel}

The strategy followed for the design of the $3 \ell$ search channel has many similarities with the $2 \ell$ channel. Three SRs are defined with the standard decay tree (SR3 $\ell$ _High, SR3 $\ell$ Int, SR $3 \ell$ Low) and the diboson background contribution is controlled and validated in a dedicated $\mathrm{CR}$ $(\mathrm{CR} 3 \ell-\mathrm{VV})$ and VR (VR3 $\ell$-VV), which contain mutually exclusive events with respect to the SRs. The initial selection of events proceeds with preselection requirements summarized in Table VI. All regions require exactly three energetic leptons with the transverse momentum of the third leading lepton in $p_{\mathrm{T}}, p_{\mathrm{T}}^{\ell_{3}}$, required to be at least $30 \mathrm{GeV}$. The regions are additionally required to have low jet activity. A same-flavor opposite-charge lepton pair is required, formed by finding the pair with invariant mass closest to the $Z$-boson mass, while the remaining (unpaired) lepton is used to construct $m_{\mathrm{T}}^{W}$. SR $3 \ell$ Low has a jet veto which makes it orthogonal to the ISR SR (SR3 $\ell$ _ISR) that is described below.

The selection requirements defining the SRs, CR and VR can be seen in Table VII. For signals targeting larger masses, and hence mass splittings between the parent and 
LSP ("high" and "intermediate" regions), the selection criteria imposed on scale quantities are tighter, with looser requirements applied to ratio values. The opposite is true as the mass splitting becomes smaller, where the selection criteria imposed on scale quantities are less stringent, since the produced objects are not expected to be too energetic; better sensitivity is obtained by applying selection criteria to ratios of quantities. Orthogonality between the CR, VR and SRs is achieved by inverting the requirement on $m_{\mathrm{T}}^{W}$ and using different transverse-mass windows.

SR3 $\ell$ Low requires no jet activity, so an orthogonal $3 e$ ISR region is defined when there are jets in the event. As with all uses of the compressed decay tree, at least one jet must be identified in the event, to populate the ISR system. For the SR3 $\ell$ ISR region all jets are associated with the ISR system. The highly energetic ISR system that accompanies the leptons reduces the contributions from fake or nonprompt (FNP) leptons and allows the relaxation of lepton $p_{\mathrm{T}}$ thresholds. The exact preselection requirements applied in the ISR regions are shown in Table VIII.

The lepton pair formation follows the same prescription used for the regions constructed with the standard decay tree. The selection criteria applied to the events after preselection are given in Table IX. The diboson CR (CR3 $\ell$ ISR-VV) is defined with an inverted $m_{\mathrm{T}}^{W}$ requirement while the corresponding VR (VR3 $\ell$ ISR-VV) is defined with a relaxed requirement on $m_{\mathrm{T}}^{W}$ and has the $p_{\mathrm{T}}^{\mathrm{CM}}$ requirement inverted.

Postfit distributions of variables from the $3 \ell$ search for selected regions, are shown in Figs. 6 and 7 for data and the different MC samples. The background component labeled "Others" refers to the processes with a Higgs boson, $t \bar{t} V$ and the nonprompt and nonisolated leptons.

\section{BACKGROUND ESTIMATION}

Several SM background processes contribute to the event counts in the signal regions. The largest backgrounds arise from dibosons and $Z+$ jets, with lesser contributions from top-quark pairs, single top quarks, tribosons and Higgs bosons. In general, these backgrounds can be classified into two categories, the irreducible backgrounds with prompt and isolated leptons (also referred to as real leptons) and genuine $E_{\mathrm{T}}^{\text {miss }}$ from neutrinos, and reducible backgrounds that contain one or more FNP lepton(s) or where experimental effects (e.g., detector mismeasurements of jets or leptons or imperfect removal of object double-counting) lead to significant "fake" $E_{\mathrm{T}}^{\mathrm{miss}}$.

An FNP lepton can originate from a semileptonic decay of a $b$ - or $c$-hadron, decays in flight of light hadrons, misidentification of a light-flavor jet, or photon conversions. In the $2 \ell$ analysis such backgrounds originate from multijet, $W+$ jets, single-top-quark and $t \bar{t}$ production events, while in the $3 \ell$ analysis there are additional contributions from $Z+$ jets and $W W$ and from any other physics process leading to less than three prompt and isolated leptons. In both analyses, this background is estimated using a datadriven technique, the matrix method [93].

This method uses two types of lepton identification criteria: "signal," corresponding to high-purity leptons and "baseline," corresponding to the definition of Sec. IV. The method makes use of the numbers of observed events containing baseline-baseline, baseline-signal, signalbaseline and signal-signal lepton pairs (ordered in $p_{\mathrm{T}}$ ) in a given SR. In the $3 \ell$ search channel the highest- $p_{\mathrm{T}}$ electron or muon is taken to be real. Simulation studies show that this is a valid assumption in $>95 \%$ of three-signal-lepton events. Knowing the probabilities for real and FNP leptons satisfying the baseline selection criteria to also satisfy the signal selection, the observed event counts with the different lepton selection criteria can be used to extract a data-driven estimate of the FNP background. The probabilities are calculated similarly to Ref. [18].

\section{A. Background estimate in the two-lepton channel}

The $Z+$ jets process can provide a large background, particularly in the low-mass and compressed SRs, due to fake $E_{\mathrm{T}}^{\text {miss }}$ from jet or lepton mismeasurements or from neutrinos in semileptonic decays of $b$ - or $c$-hadrons. These effects are difficult to model in simulation, so instead $\gamma+$ jets events in data are used to extract the $E_{\mathrm{T}}^{\text {miss }}$ shape in $Z+$ jets events. Similar methods were employed in searches for SUSY in events with two leptons, jets, and large $E_{\mathrm{T}}^{\text {miss }}$ in ATLAS [94] and CMS $[95,96]$. The $E_{\mathrm{T}}^{\text {miss }}$ shape is extracted from a data control sample of $\gamma+$ jets events, which have a topology similar to $Z+$ jets events, recorded using a set of single-photon triggers. The events selected with prescaled triggers correspond to photon $p_{\mathrm{T}}<140 \mathrm{GeV}$ and these events are weighted with the corresponding trigger prescale factor. Corrections for the different $\gamma$ versus $Z$-boson $p_{\mathrm{T}}$ distributions and different momentum resolutions for electrons, muons, and photons are applied. Backgrounds from $W \gamma$ and $Z \gamma$ production, which contain a photon and genuine $E_{\mathrm{T}}^{\text {miss }}$ from neutrinos, are subtracted using MC simulation that is normalized to data in a $V \gamma$ control region containing a selected lepton and photon. The $V \gamma$ normalization factor is found to be equal to $0.79 \pm 0.79$.

To model quantities that depend on the individual lepton momenta, a $m_{\ell \ell}$ value is assigned to each $\gamma+$ jets event by sampling from $m_{\ell \ell}$ distributions (parametrized as a function of boson $p_{\mathrm{T}}$ and the component of $E_{\mathrm{T}}^{\text {miss }}$ that is parallel to the boson $p_{\mathrm{T}}$ ) extracted from $Z+$ jets simulation. Each $\gamma+$ jets event is boosted to the rest frame of the emulated $Z$ boson and the photon is split into two pseudo-leptons, assuming isotropic decays in the rest frame. In all the twolepton SRs (except for SR2 $\ell$ LLow) the $Z+$ jets background is directly estimated by weighting appropriately the $\gamma+$ jets events surviving the SR selections. In SR $2 \ell$ Low, the direct $Z+$ jets background estimation lacks statistical 
precision due to the high prescale factors of the triggers used to select $\gamma+$ jets events with low momentum $\left(p_{\mathrm{T}}^{\gamma}<100 \mathrm{GeV}\right)$, as opposed to the other SRs whose definitions, including an ISR-jet requirement, are such that events with a large dilepton system $p_{\mathrm{T}}\left(p_{\mathrm{T}}^{\ell \ell}\right)$ are selected. Due to this, an alternative approach is used for the $Z+$ jets estimate in the low-mass SR, which relies on the robust $\gamma+$ jets estimate of high- $p_{\mathrm{T}}^{\ell \ell}\left(p_{\mathrm{T}}^{\ell \ell}>100 \mathrm{GeV}\right)$ events. The $\gamma+$ jets template is used to directly estimate the high- $p_{\mathrm{T}}^{\ell \ell}$ $Z+$ jets component of SR $2 \ell$ Low while the low- $p_{\mathrm{T}}^{\ell \ell}$ $\left(p_{\mathrm{T}}^{\ell \ell}<100 \mathrm{GeV}\right) Z+$ jets contribution is estimated by using a transfer factor defined as the ratio of low- $p_{\mathrm{T}}^{\ell \ell}$ to high- $p_{\mathrm{T}}^{\ell \ell}$ events and is calculated from an orthogonal sample with an inverted $H_{4,1}^{\mathrm{PP}}$ requirement. The ratio is found to be $3.9 \pm 2.1$, while the high- $p_{\mathrm{T}}^{\ell \ell} Z+$ jets estimate is $1.29 \pm 0.5$. The uncertainties quoted are statistically only.

To validate the method, as well as to check the modeling of other SM backgrounds, validation regions are defined for each SR. The definitions of these regions (VR2 $\ell-\mathrm{VV}$, VR2 $\ell$-Top, VR $2 \ell$ _High-Zjets and VR2 $\ell$ _Low-Zjets) are given for the standard decay tree in Table III and (VR2 $\ell$ _ISR-VV, VR2 $\ell$ _ISR-Top and VR2 $\ell$ _ISR-Zjets) for the compressed decay tree in Table V. The VRs targeting the validation of the $Z+$ jets background estimation have an inverted dijet mass requirement with respect to the corresponding SR definitions as well as having some other selection criteria relaxed. In this way a potential signal contribution is rejected while the regions remain close but orthogonal to the SR selections.

As described in Sec. VII, the background contributions from $W t+t \bar{t}$ and $V V$ are normalized to data in dedicated $C R s$ and the extracted normalization factors from the fit are validated in orthogonal regions. The $V V$ process in the SRs has contributions from all diboson processes producing at least two leptons in the final state. The dominant diboson process in $\mathrm{SR} 2 \ell \_$High and $\mathrm{SR} 2 \ell \_$Int is $Z Z \rightarrow \ell \ell \nu \nu$ with a smaller contribution from $W Z \rightarrow \ell \nu \ell \ell$. The picture changes with lower $\tilde{\chi}_{1}^{ \pm} / \tilde{\chi}_{2}^{0}$ masses and smaller mass splitting; in SR2 $\ell$ Low the dominant component is $W W \rightarrow$ $\ell \nu \ell \nu$ followed by $W Z \rightarrow \ell \nu \ell \ell$ while in SR2 $\ell$ ISR the dominant contribution is from $W Z \rightarrow \ell \nu \ell \ell$ and to a lesser extent from $Z Z \rightarrow \ell \ell \nu \nu$. The semihadronic decays of dibosons, for example $Z V \rightarrow \ell \ell q q$, are accounted for by the $\gamma+$ jets template since they do not lead to genuine $E_{\mathrm{T}}^{\text {miss }}$ in the event. The CRs are designed to have compositions, in terms of diboson processes, similar to their respective SRs.

The two-lepton diboson and top CRs defined with the standard decay tree do not contain an explicit selection to make them orthogonal to their respective compressed CRs. However, the two decay trees of the RJR method, by construction, probe different event topologies, hence they select events where the overlap is designed to be insignificant. For the top CR the overlap is less than $1 \%$ while for the diboson CR it is smaller than $3 \%$. Since the impact of this effect is negligible in comparison with the background uncertainties, it is not considered in the remainder of the analysis.

The normalization factors obtained from the background-only fit for $W t+t \bar{t}$ and $V V$ for the selections applied to the standard (compressed) decay tree are $0.91 \pm$ 0.23 and $0.91 \pm 0.13(0.99 \pm 0.12$ and $0.94 \pm 0.18)$, respectively, where the uncertainties are dominated by the statistical uncertainty. The background fit results are summarized in Tables X and XI for the CRs and VRs, respectively. The data are consistent with the expected background in all validation regions.

\section{B. Background estimate in the three-lepton channel}

The irreducible background in the $3 \ell$ channel is dominated by SM $W Z$ diboson production. The shape of the diboson background is taken from simulation but normalized to data in

TABLE X. Background fit results for the $2 \ell \mathrm{CRs}$. The normalization factors for $W t+t \bar{t}$ and $V V$ for the standard and compressed decay trees are different and are extracted from separate fits. The nominal predictions from MC simulation are given for comparison for the $W t+t \bar{t}$ and $V V$ backgrounds. The "Other" category contains the contributions from Higgs boson processes, $V \gamma, V V V, t \bar{t} V$ and nonprompt and nonisolated lepton production. The dashes indicate that these backgrounds are negligible and are included in the category "Other." Combined statistical and systematic uncertainties are given. The individual uncertainties can be correlated and do not necessarily add in quadrature to the total systematic uncertainty.

\begin{tabular}{lcccc}
\hline \hline Region & CR2 $\ell$-VV & CR2 $\ell$-Top & CR2 $\ell$ _ISR-VV & CR2 $\ell$ ISR-Top \\
\hline Observed events & 60 & 97 & 28 & 93 \\
Total (postfit) SM events & $60 \pm 8$ & $97 \pm 10$ & $28 \pm 5$ & $93 \pm 10$ \\
Other & $3.5 \pm 0.3$ & $1.4 \pm 0.3$ & $0.72 \pm 0.31$ & $0.50 \pm 0.15$ \\
Fit output, $W t+t \bar{t}$ & $\ldots$ & $60 \pm 11$ & $\ldots$ & $90 \pm 10$ \\
Fit output, $V V$ & $57 \pm 8$ & $31 \pm 15$ & $27 \pm 5$ & $0.99 \pm 0.31$ \\
$Z+$ jets & $\ldots$ & 66 & $\ldots$ & $2.1 \pm 1.0$ \\
Fit input, $W t+t \bar{t}$ & $\ldots 2$ & 4.4 & 29 & 91 \\
Fit input, $V V$ & & & $\ldots$ & 1.1 \\
\hline \hline
\end{tabular}


TABLE XI. Expected and observed yields from the background fit for the $2 \ell$ VRs. The nominal predictions from MC simulation are given for comparison for the $W t+t \bar{t}$ and $V V$ backgrounds. The "Other" category contains the contributions from Higgs boson processes, $V \gamma, V V V, t \bar{t} V$ and nonprompt and nonisolated lepton production. The dashes indicate that these backgrounds are negligible and are included in the category "Other." Combined statistical and systematic uncertainties are given. The individual uncertainties can be correlated and do not necessarily add in quadrature to the total systematic uncertainty.

\begin{tabular}{lccccccc}
\hline \hline Region & VR2 $\ell$ Low-Zjets & VR2 $\ell$ High-Zjets & VR2 $\ell$-VV & VR2 $\ell$-Top & VR2 $\ell$ ISR-VV & VR2 $\ell$ ISR-Top VR2 $\ell$ ISR-Zjets \\
\hline Observed events & 263 & 77 & 72 & 491 & 13 & 113 & 248 \\
Total (postfit) & $261 \pm 130$ & $69 \pm 26$ & $61 \pm 13$ & $423 \pm 105$ & $12 \pm 4$ & $110 \pm 18$ & $310 \pm 100$ \\
$\quad$ SM events & & & & & & & \\
Other & $3.5 \pm 1.5$ & $0.25_{-0.25}^{+0.62}$ & $0.80 \pm 0.09$ & $2.3 \pm 0.4$ & $4.2 \pm 0.5$ & $0.68 \pm 0.22$ & $3.0 \pm 0.6$ \\
Fit output, $W t+t \bar{t}$ & $15 \pm 5$ & $1.7 \pm 0.7$ & $12 \pm 4$ & $415 \pm 105$ & $\ldots$ & $107 \pm 18$ & $40 \pm 8$ \\
Fit output, $V V$ & $30 \pm 7$ & $16 \pm 3$ & $40 \pm 13$ & $3.7 \pm 0.9$ & $7.9 \pm 3.6$ & $0.97 \pm 0.25$ & $67 \pm 15$ \\
$Z+$ jets & $210 \pm 130$ & $51 \pm 25$ & $8.4 \pm 4.1$ & $2.4 \pm 1.2$ & $\ldots$ & $1.6 \pm 0.8$ & $200 \pm 100$ \\
Fit input, $W t+t \bar{t}$ & 16 & 1.9 & 13 & 455 & $\ldots$ & 108 & 41 \\
Fit input, $V V$ & 33 & 17 & 43 & 4.1 & 8.4 & 1.1 & 71 \\
\hline \hline
\end{tabular}

TABLE XII. Expected and observed yields from the background fit for the $3 \ell$ CRs and VRs. The normalization factors for $V V$ for the standard and compressed decay trees are different and are extracted from separate fits. The nominal predictions from MC simulation are given for comparison for the $V V$ background. The "Other" category contains the contributions from Higgs boson processes, $t \bar{t} V$ and nonprompt and nonisolated lepton production. Combined statistical and systematic uncertainties are given. The individual uncertainties can be correlated and do not necessarily add in quadrature to the total systematic uncertainty.

\begin{tabular}{lcccc}
\hline \hline Region & CR3 $\ell$-VV & VR3 $\ell$-VV & CR3 $\ell$ ISR-VV & VR3 $\ell$ ISR-VV \\
\hline Observed events & 331 & 160 & 98 & 83 \\
Total (postfit) SM events & $331 \pm 18$ & $159 \pm 38$ & $98 \pm 10$ & $109 \pm 24$ \\
Other & $52 \pm 13$ & $5.6 \pm 1.2$ & $4.4 \pm 1.2$ & $7.1 \pm 1.6$ \\
Tribosons & $1.1 \pm 0.1$ & $0.44 \pm 0.03$ & $0.22 \pm 0.14$ & $0.42 \pm 0.04$ \\
Fit output, $V V$ & $278 \pm 18$ & $153 \pm 38$ & $93 \pm 10$ & $102 \pm 24$ \\
Fit input, $V V$ & 255 & 140 & 83 & 90 \\
\hline \hline
\end{tabular}

dedicated CRs. The normalization factors extracted from the background-only fit are found to be $1.09 \pm 0.10$ and $1.13 \pm$ 0.13 for the standard and compressed decay tree selections, respectively. The results of the background estimates are validated in a set of dedicated VRs. Other background sources such as $V V V, t \bar{t} V$ and processes with a Higgs boson contributing to the irreducible background are taken from simulation. A summary of the background fit results for the $3 \ell$ CRs and VRs is given in Table XII.

Similar to the two-lepton CR design, the three-lepton diboson CR defined with the standard decay tree does not contain an explicit selection to make it orthogonal to its respective compressed CR. The overlap is less than $0.5 \%$. Since the impact of this effect is negligible in comparison with the background uncertainties, it is not considered in the remainder of the analysis.

\section{SYSTEMATIC UNCERTAINTIES}

Several sources of experimental and theoretical systematic uncertainties are considered in the SM background estimates and signal expectations and are included in the profile likelihood fits described in Sec. V. The systematic uncertainties that are considered are related to the jet energy scale and resolution, the modeling of $E_{\mathrm{T}}^{\text {miss }}$ in the simulation, the lepton reconstruction and identification, the $V V$ theoretical modeling uncertainties, the nonprompt lepton background estimation and the data-driven $Z+$ jets estimate. The effects of these uncertainties are evaluated for all signal event samples and background processes. The normalization of the $W t+t \bar{t}$ and $V V$ background predictions is extracted in dedicated control regions and the systematic uncertainties thus only affect the extrapolation to the SRs. The statistical uncertainty due to the number of events in the MC samples is also included. The systematic uncertainty associated with the pileup reweighting of the simulated events is also considered and found to have a negligible impact on the final results.

The jet energy scale and resolution uncertainties are derived as a function of the $p_{\mathrm{T}}$ and $\eta$ of the jet, as well as of the pileup conditions and the jet flavor composition of the selected jet sample. They are determined using a combination of simulated events and data samples, through 
TABLE XIII. Summary of the main systematic uncertainties and their impact (in \%) on the total SM background prediction in each of the $2 \ell$ SRs. The total systematic uncertainty can be different from the sum in quadrature of individual sources due to the correlations between them resulting from the fit to the data.

\begin{tabular}{|c|c|c|c|c|}
\hline Signal region & SR $2 \ell \_H i g h$ & SR $2 \ell$ Int & SR2 $\ell$ _Low & SR $2 \ell$ ISR \\
\hline Total uncertainty [\%] & 42 & 38 & 70 & 103 \\
\hline$Z+$ jets data-driven estimate & 42 & 31 & 69 & 96 \\
\hline$V V$ theoretical uncertainties & 28 & 27 & 6 & 34 \\
\hline MC statistical uncertainties & 16 & 12 & 5 & 9 \\
\hline$V V$ fitted normalization & 13 & 14 & 2 & 16 \\
\hline FNP leptons & $\ldots$ & 5 & 13 & 12 \\
\hline Jet energy resolution & 5 & 10 & 4 & 3 \\
\hline Jet energy scale & 1 & 2 & $<1$ & 3 \\
\hline$E_{\mathrm{T}}^{\mathrm{miss}}$ modeling & 3 & 4 & $<1$ & $<1$ \\
\hline$t \bar{t}$ fitted normalization & $<1$ & $<1$ & 2 & 2 \\
\hline Lepton reconstruction/identification & $<1$ & $<1$ & $<1$ & $<1$ \\
\hline
\end{tabular}

measurements of the jet response balance in multijet, $Z+$ jets and $\gamma+$ jets events [83].

The systematic uncertainties related to the modeling of $E_{\mathrm{T}}^{\text {miss }}$ in the simulation are estimated by propagating the uncertainties in the energy and momentum scale of each of the physics objects, as well as the uncertainties in the softterm resolution and scale [97].

The remaining detector-related systematic uncertainties, such as those in the lepton reconstruction efficiency, $b$-tagging efficiency $[98,99]$, lepton energy scale, energy resolution and in the modeling of the trigger [75,76], are included but are found to be negligible in all channels.

The uncertainties arising from the modeling of diboson events in simulation are estimated by varying the renormalization, factorization and merging scales used to generate the samples, as well as the PDFs.

In the $2 \ell$ channel, uncertainties in the data-driven $Z+$ jets estimate are calculated following the methodology used in Ref. [94]. An additional uncertainty is based on the difference between the expected background yield from the nominal method (which produces 6.3 events in SR2 $\ell$ Low and 0.1 events in SR2 $\ell$ ISR) and from a second method implemented as a cross-check, which extracts the dijet mass shape from data validation regions, normalizes the shape to the sideband regions of the SRs, and extrapolates the background into the $W$ mass region. The $Z+$ jets background estimations obtained from the sideband method are 5.9 and 0.2 events for SR $2 \ell$ Low and SR $2 \ell$ ISR, respectively. Moreover, a $100 \%$ uncertainty in the $V \gamma$ normalization factor is included. To cover any statistical limitations on the $Z+$ jets estimate that may be present in SR $2 \ell$ _ISR, an upper limit on the $Z+$ jets estimate is considered as an additional systematic uncertainty. The upper limit is calculated by multiplying the sum of the nominal $Z+$ jets background estimate, adding the statistical uncertainty, with the ratio of low- $p_{\mathrm{T}}^{\ell \ell}$ to high- $p_{\mathrm{T}}^{\ell \ell}$ events calculated with a looser requirement on $p_{\mathrm{TI}}^{\mathrm{CM}}$. This is the dominant uncertainty in the ISR region and accounts for $95 \%$ of the total uncertainty in the $Z+$ jets estimate.

Systematic uncertainties are also assigned to the estimated background from FNP leptons in both the $2 \ell$ and $3 \ell$ channels to account for potentially different compositions

TABLE XIV. Summary of the main systematic uncertainties and their impact (in \%) on the total SM background prediction in each of the $3 \ell$ SRs. The total systematic uncertainty can be different from the sum in quadrature of individual sources due to the correlations between them resulting from the fit to the data.

\begin{tabular}{lcccc}
\hline \hline Signal region & SR3 $\ell$ _High & SR3 $\ell$ Int & SR3 $\ell$ Low & SR3 $\ell$ ISR \\
\hline Total uncertainty [\%] & 44 & 22 & 19 & 26 \\
$V V$ theoretical uncertainties & 18 & 9 & 12 & 19 \\
MC statistical uncertainties & 37 & 17 & 8 & 10 \\
$V V$ fitted normalization & 8 & 7 & 9 & 11 \\
FNP leptons & 7 & $<1$ & 3 & 5 \\
Jet energy resolution & 4 & $<1$ & 7 & 3 \\
Jet energy scale & 7 & $<1$ & 1 & 3 \\
$E_{\mathrm{T}}^{\text {miss }}$ modeling & 2 & 4 & 2 & 4 \\
Lepton reconstruction/identification & 3 & & 2 \\
\hline \hline
\end{tabular}


TABLE XV. Expected and observed yields from the background-only fit for the $2 \ell$ SRs. The errors shown are the statistical plus systematic uncertainties. Uncertainties in the predicted background event yields are quoted as symmetric, except where the negative error reaches down to zero predicted events, in which case the negative error is truncated.

\begin{tabular}{lcccc}
\hline \hline Signal region & SR2 $\ell$ _High & SR2 $\ell$ Int & SR2 $\ell$ Low & SR2 $\ell$ _ISR \\
\hline Total observed events & 0 & 1 & 19 & 11 \\
Total background events & $1.9 \pm 0.8$ & $2.4 \pm 0.9$ & $8.4 \pm 5.8$ & $2.7_{-2.7}^{+2.8}$ \\
Other & $0.02 \pm 0.01$ & $0.05_{-0.05}^{+0.12}$ & $0.02_{-0.02}^{+1.07}$ & $0.06_{-0.06}^{+0.33}$ \\
Fit output, $W t+t \bar{t}$ & $0.00 \pm 0.00$ & $0.00 \pm 0.00$ & $0.57 \pm 0.20$ & $0.28_{-0.28}^{+0.34}$ \\
Fit output, $V V$ & $1.8 \pm 0.7$ & $2.4 \pm 0.8$ & $1.5 \pm 0.9$ & $2.3 \pm 1.1$ \\
$Z+$ jets & $0.07_{-0.07}^{+0.78}$ & $0.00_{-0.00}^{+0.74}$ & $6.3 \pm 5.8$ & $0.10_{-0.10}^{+2.58}$ \\
Fit input, $W t+t \bar{t}$ & 0.00 & 0.00 & 0.63 & 0.28 \\
Fit input, $V V$ & 1.9 & 2.6 & 1.6 & 2.4 \\
\hline \hline
\end{tabular}

(heavy flavor, light flavor or conversions) between the signal and control regions. An additional uncertainty is associated with the subtraction of prompt leptons from this CR using simulation.

A summary of the dominant uncertainties in the $2 \ell$ SRs is shown in Table XIII. The uncertainties with the largest impact in these SRs are those in the data-driven $Z+$ jets estimate, followed by the $V V$ modeling uncertainties, the statistical uncertainties in the MC background samples and the uncertainty in the fitted normalization factor for $V V$ related to the number of events in the corresponding CRs.

A similar summary of the systematic uncertainties impacting the $3 \ell \mathrm{SRs}$ is given in Table XIV. These are dominated by the statistical uncertainties in the MC background samples, the modeling uncertainties in the $V V$ processes and the uncertainties related to the fitted normalization factors for $V V$.

\section{RESULTS AND INTERPRETATION}

The observed numbers of events in the $2 \ell$ channel are compared with the expected background contributions in Table XV and Fig. 8; those in the $3 \ell$ channel are shown in Table XVI and Fig. 9. No significant excesses above the SM expectation are observed in the SRs targeting intermediate- and high-mass signal models. An excess of events above the background estimate is observed in each of the four low-mass and ISR signal regions. To quantify the level of agreement of the observed data with the SM expectations, a model-independent fit is performed separately for each SR. The results of this fit for the $2 \ell$ and $3 \ell$ searches are given in Table XVII.

Selected kinematic distributions in the low-mass and ISR regions for the $2 \ell$ and $3 \ell$ selections after applying all the selection requirements defining these SRs are shown in

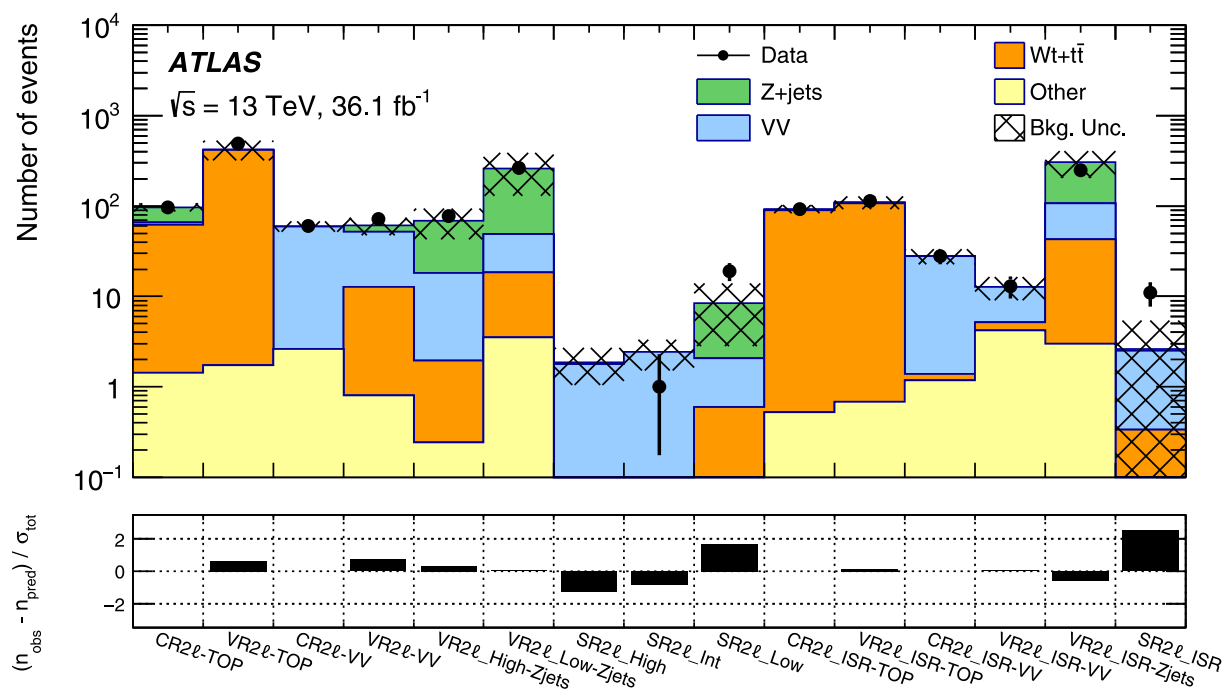

FIG. 8. The observed and expected SM background yields in the CRs, VRs and SRs considered in the $2 \ell$ channel. The statistical uncertainties in the background prediction are included in the uncertainty band, as well as the experimental and theoretical uncertainties. The bottom panel shows the difference in standard deviations between the observed and expected yields. 
TABLE XVI. Expected and observed yields from the background-only fit for the $3 \ell$ SRs. The errors shown are the statistical plus systematic uncertainties. Uncertainties in the predicted background event yields are quoted as symmetric, except where the negative error reaches down to zero predicted events, in which case the negative error is truncated.

\begin{tabular}{lcccc}
\hline \hline Signal region & SR3 $\ell$ _High & SR3 $\ell \_$Int & SR3 $\ell \_$Low & SR3 $\ell$ ISR \\
\hline Total observed events & 2 & 1 & 20 & 12 \\
Total background events & $1.1 \pm 0.5$ & $2.3 \pm 0.5$ & $10 \pm 2$ & $3.9 \pm 1.0$ \\
Other & $0.03_{-0.03}^{+0.07}$ & $0.04 \pm 0.02$ & $0.02_{-0.02}^{+0.34}$ & $0.06_{-0.06}^{+0.019}$ \\
Triboson & $0.19 \pm 0.07$ & $0.32 \pm 0.06$ & $0.25 \pm 0.03$ & $0.08 \pm 0.04$ \\
Fit output, $V V$ & $0.83 \pm 0.39$ & $1.9 \pm 0.5$ & $10 \pm 2$ & $3.8 \pm 1.0$ \\
Fit input, $V V$ & 0.76 & 1.8 & 9.2 & 3.4 \\
\hline \hline
\end{tabular}

Figs. 10 and 11, respectively. In all figures a SUSY signal benchmark model is shown for illustration. This simplified model assumes $m_{\tilde{\chi}_{1}^{ \pm} / \tilde{\chi}_{2}^{0}}=200 \mathrm{GeV}$ and $m_{\tilde{\chi}_{1}^{0}}=100 \mathrm{GeV}$, and was used to optimize the event selection criteria for the low-mass and ISR SRs.

With the complementarity of the $3 \ell$ low-mass and ISR regions, a study of events that fall in either one or the other is possible. Many of the discriminating variables are specific to the decay trees, hence events in the ISR and low-mass SRs cannot be displayed together in these observables. Figure 12 shows the transverse mass distribution, calculated using the unpaired lepton prior to the selection imposed on this variable, for events passing the $3 e$ low-mass [12(a)] and the $3 e$ ISR SR requirements [12(b)]. These distributions show events with no additional jet activity, along with those including a jet identified as emanating from an ISR system. In both figures there is an excess of events with transverse mass above the minimum value of $100 \mathrm{GeV}$ required in both $\mathrm{SR} 3 \ell$ Low and SR $3 \ell$ _ISR.

Exclusion limits for simplified models, in which pairs of $\tilde{\chi}_{1}^{ \pm} \tilde{\chi}_{2}^{0}$ decay with $100 \%$ branching ratio into $W / Z$ vector bosons, are shown in Fig. 13. Figures 13(a) and 13(b) show the exclusion limits obtained from the $2 \ell$ and $3 \ell$ channels respectively and after selecting the SR with the highest expected sensitivity for each signal-model assumption. The low-mass and ISR regions are statistically combined. Figure 13(c) corresponds to the statistical combination of the $2 \ell$ and $3 \ell$ search channels. The combination proceeds by statistically combining the SRs of the two channels which target the same region in the two-dimensional parameter space (e.g., SR $2 \ell$ _High with SR $3 \ell_{\text {_High }}$ ) since they contain mutually exclusive events. Once the statistical combination is performed then the combined SR producing the best expected $\mathrm{CL}_{\mathrm{s}}$ value for each model assumption is chosen. The last step is needed since the high-, intermediate- and

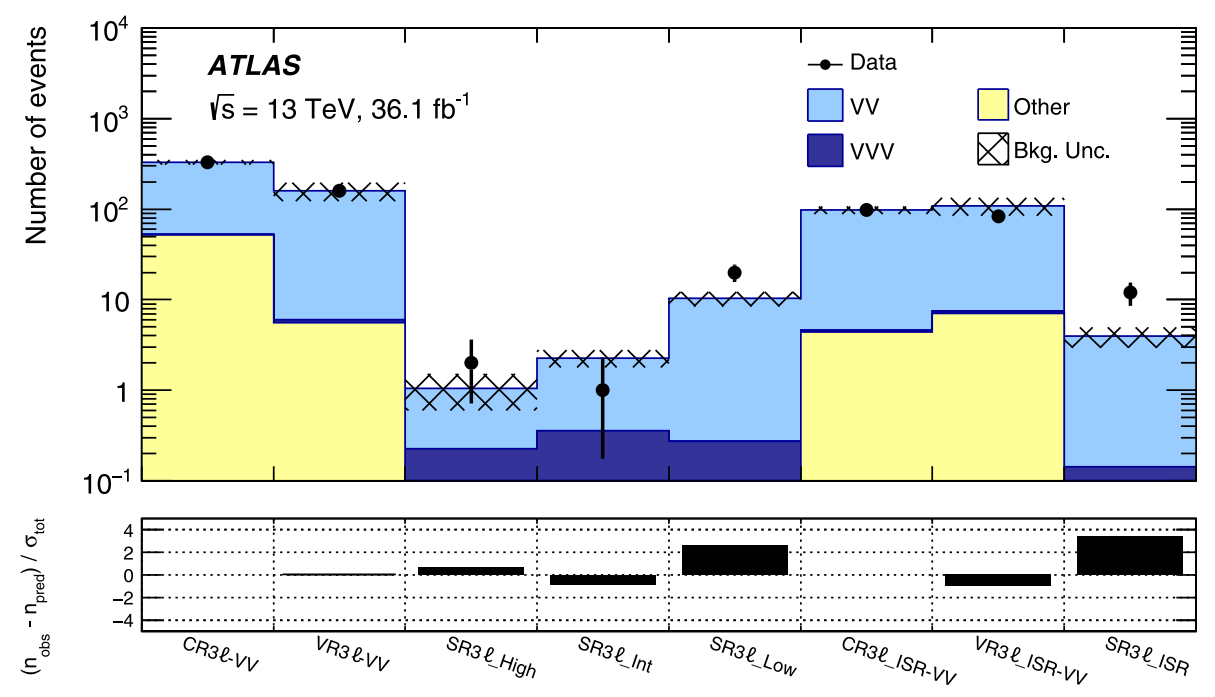

FIG. 9. The observed and expected SM background yields in the CRs, VRs and SRs considered in the $3 \ell$ channel. The statistical uncertainties in the background prediction are included in the uncertainty band, as well as the experimental and theoretical uncertainties. The bottom panel shows the difference in standard deviations between the observed and expected yields. 
TABLE XVII. Model-independent fit results for all SRs. The first column shows the SRs, the second and third columns show the $95 \%$ C.L. upper limits on the visible cross section $\left(\langle\epsilon \sigma\rangle_{\text {obs }}^{95}\right)$ and on the number of signal events $\left(S_{\mathrm{obs}}^{95}\right)$. The fourth column $\left(S_{\text {exp }}^{95}\right)$ shows the $95 \%$ C.L. upper limit on the number of signal events, given the expected number (and $\pm 1 \sigma$ excursions of the expectation) of background events. The last column indicates the discovery $p_{0}$-value and its associated significance $(Z)$.

\begin{tabular}{lcccc}
\hline \hline Signal region & $\langle\epsilon \sigma\rangle_{\mathrm{obs}}^{95}[\mathrm{fb}]$ & $S_{\mathrm{obs}}^{95}$ & $S_{\mathrm{exp}}^{95}$ & $p_{0}(Z)$ \\
\hline SR3 $\ell$ _ISR & 0.42 & 15.3 & $6.9_{-2.2}^{+3.1}$ & $0.001(3.02)$ \\
SR2 $\ell$ ISR & 0.43 & 15.4 & $9.7_{-2.5}^{+3.6}$ & $0.02(1.99)$ \\
SR3 $\ell$ _Low & 0.53 & 19.1 & $9.5_{-1.8}^{+4.2}$ & $0.016(2.13)$ \\
SR2 $\ell$ _Low & 0.66 & 23.7 & $16.1_{-4.3}^{+6.3}$ & $0.08(1.39)$ \\
SR3 $\ell$ Int & 0.09 & 3.3 & $4.4_{-1.5}^{+2.5}$ & $0.50(0.00)$ \\
SR2 $\ell$ Int & 0.09 & 3.3 & $4.6_{-1.5}^{+2.6}$ & $0.50(0.00)$ \\
SR3 $\ell$ High & 0.14 & 5.0 & $3.9_{-1.3}^{+2.3}$ & $0.23(0.73)$ \\
SR2 $\ell$ High & 0.09 & 3.2 & $4.0_{-1.2}^{+2.3}$ & $0.50(0.00)$ \\
\hline \hline
\end{tabular}

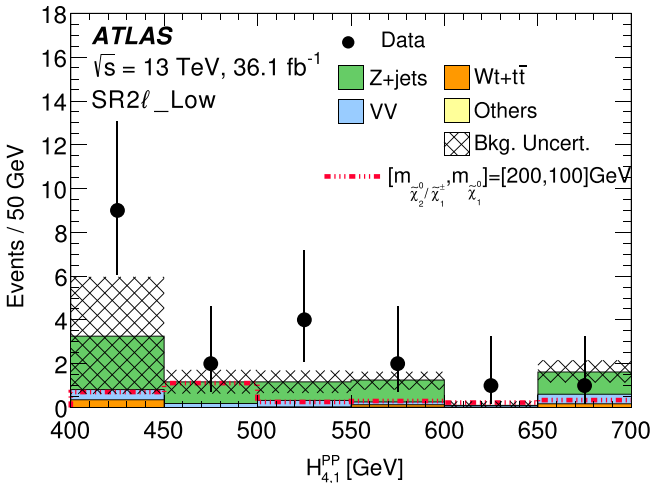

(a)

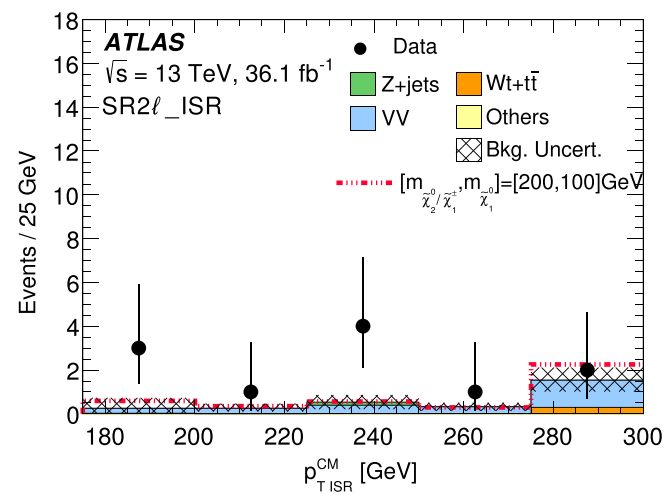

(c) low-mass SRs have event overlap while the low-mass and ISR SRs are mutually exclusive and can be statistically combined. Finally, Fig. 13(d) compares the expected and observed exclusion limits obtained from the recursive jigsaw approach with those described in Ref. [18].

The current results extend the sensitivity and exclusion limits in the high- and intermediate-mass-splitting regions compared to those from Ref. [18]. However, the low-mass region where the mass splitting is $\approx 100 \mathrm{GeV}$ cannot be excluded due to the observed excess of events. The results in this region are of interest as they show an apparent disagreement with those quoted in Ref. [18] with similar sensitivity to this simplified model. The observed data

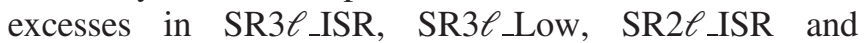
SR2 $\ell$ Low have associated significances of 3.0, 2.1, 2.0 and 1.4 standard deviations, respectively. As a result of these deviations from expectation the exclusion curves in Fig. 13 demonstrate that there are regions where an exclusion would be expected but cannot be achieved with

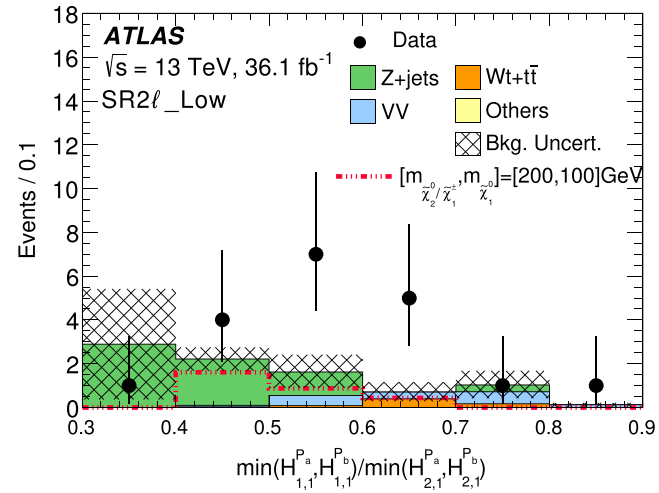

(b)

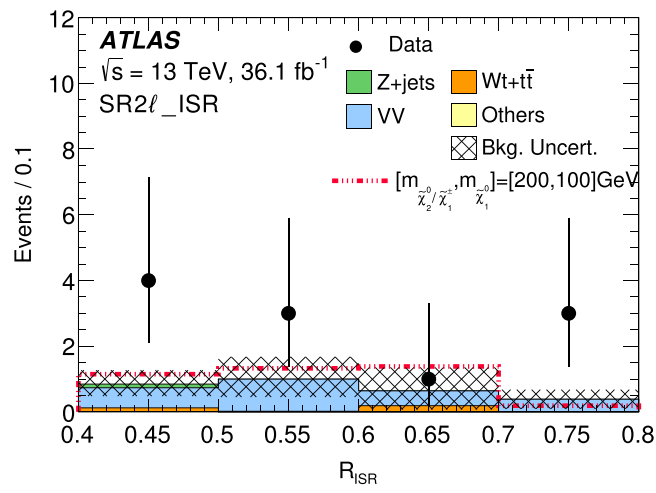

(d)

FIG. 10. Distributions of kinematic variables in the signal regions for the $2 \ell$ channels after applying all selection requirements. The histograms show the postfit background predictions. The last bin includes the overflow. The FNP contribution is estimated from a datadriven technique and is included in the category "Others." Distributions for (a) $H_{4,1}^{\mathrm{PP}}$ and (b) $\min \left(H_{1,1}^{\mathrm{P}_{\mathrm{a}}}, H_{1,1}^{\mathrm{P}_{\mathrm{b}}}\right) / \min \left(H_{2,1}^{\mathrm{P}_{\mathrm{a}}}, H_{2,1}^{\mathrm{P}_{\mathrm{b}}}\right)$ in $\mathrm{SR} 2 \ell$ Low, (c) $p_{\mathrm{TISR}}^{\mathrm{CM}}$ and (d) $R_{\mathrm{ISR}}$ in SR2 $\ell$ ISR are plotted. The hatched (black) error bands indicate the combined theoretical, experimental and MC statistical uncertainties. The expected distribution for a benchmark signal model, normalized to the NLO + NLL cross section (Sec. III) times integrated luminosity, is also shown for comparison. 


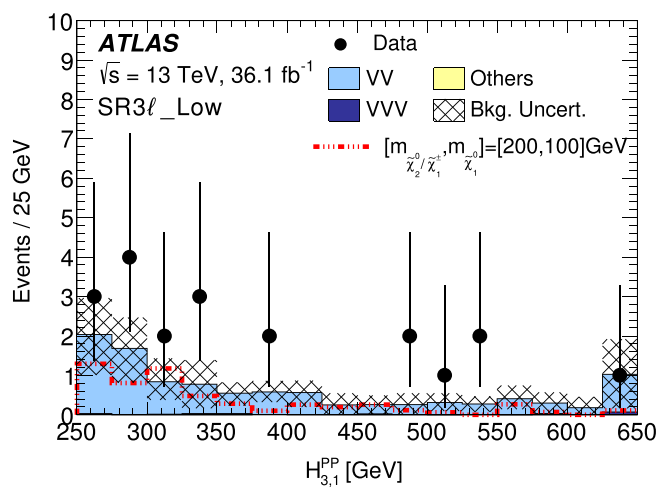

(a)

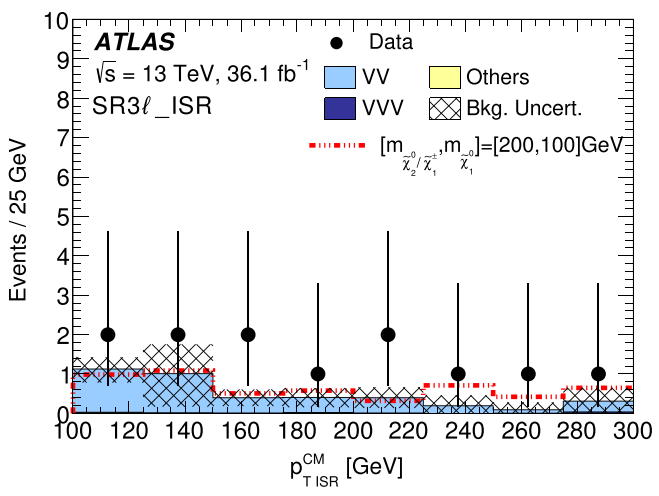

(c)

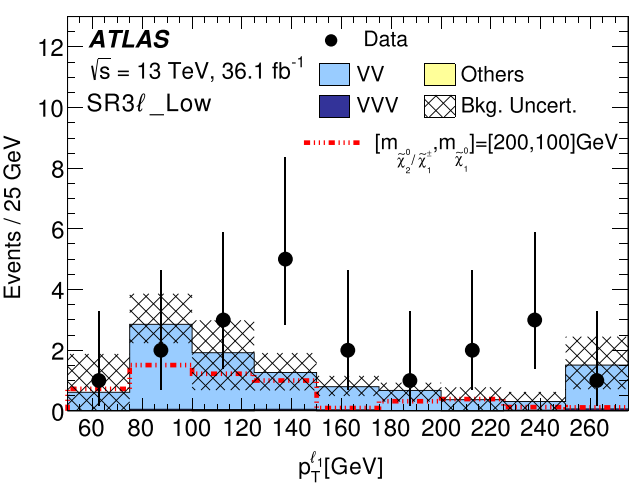

(b)

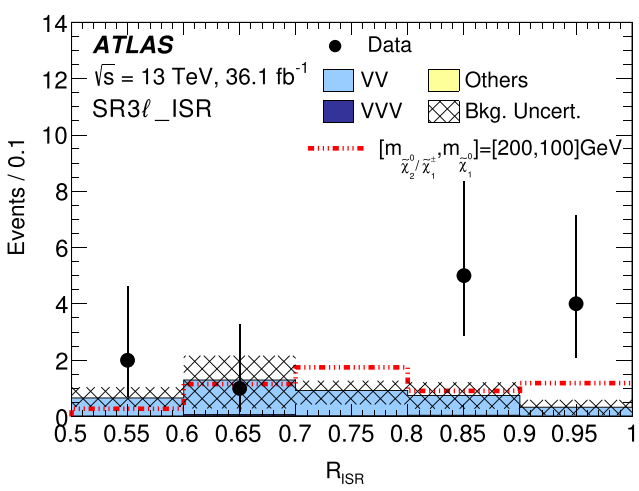

(d)

FIG. 11. Distributions of kinematic variables in the signal regions for the $3 \ell$ channels after applying all selection requirements. The histograms show the postfit background predictions. The last bin includes the overflow. The FNP contribution is estimated from a datadriven technique and is included in the category "Others." Distributions for (a) $H_{3,1}^{\mathrm{PP}}$ and (b) $p_{\mathrm{T}}^{\ell_{1}}$ in SR $3 \ell$ Low, (c) $p_{\mathrm{TISR}}^{\mathrm{CM}}$ and (d) $R_{\mathrm{ISR}}$ in SR $3 \ell$ _ISR are plotted. The hatched (black) error bands indicate the combined theoretical, experimental and MC statistical uncertainties. The expected distribution for a benchmark signal model, normalized to the NLO + NLL cross section (Sec. III) times integrated luminosity, is also shown for comparison.

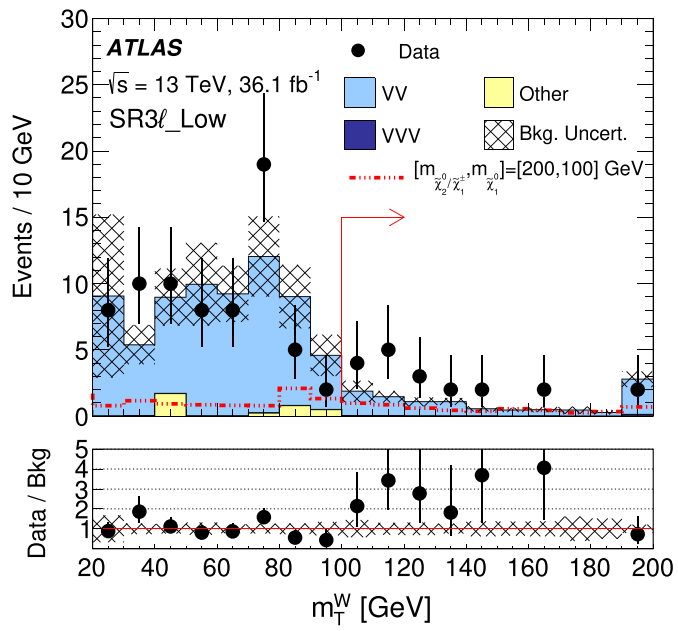

(a)

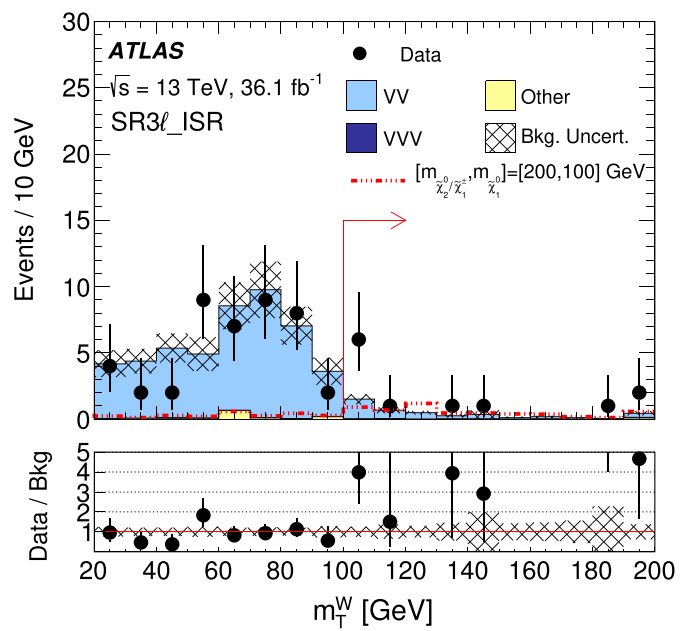

(b)

FIG. 12. The transverse mass of the unpaired lepton for events falling in either (a) SR3 $\ell$ Low or (b) SR $3 \ell$ ISR prior to the selection placed on this variable. The solid red line and arrow indicates the requirement defining these SRs. The last bin includes the overflow. The FNP contribution is estimated from a data-driven technique and is included in the category "Others." The hatched (black) error bands indicate the combined theoretical uncertainties on $V V$, experimental and $\mathrm{MC}$ statistical uncertainties. The expected distribution for a benchmark signal model, normalized to the NLO + NLL cross section (Sec. III) times integrated luminosity, is also shown for comparison. 


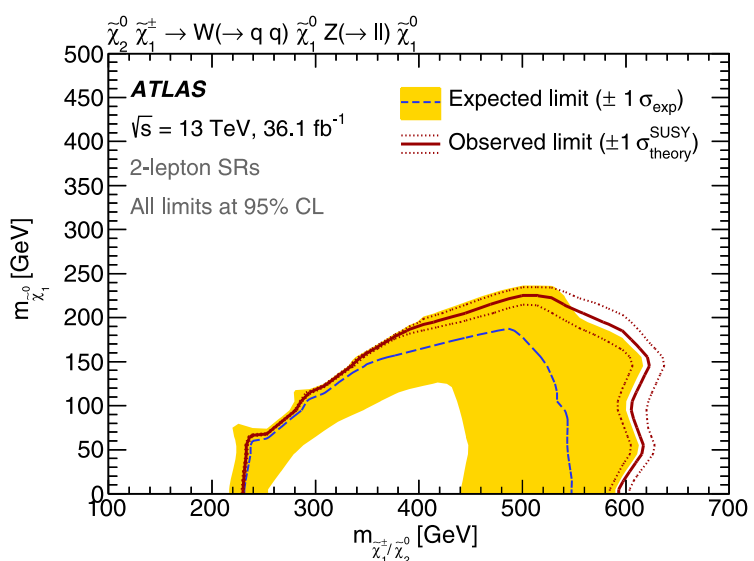

(a)

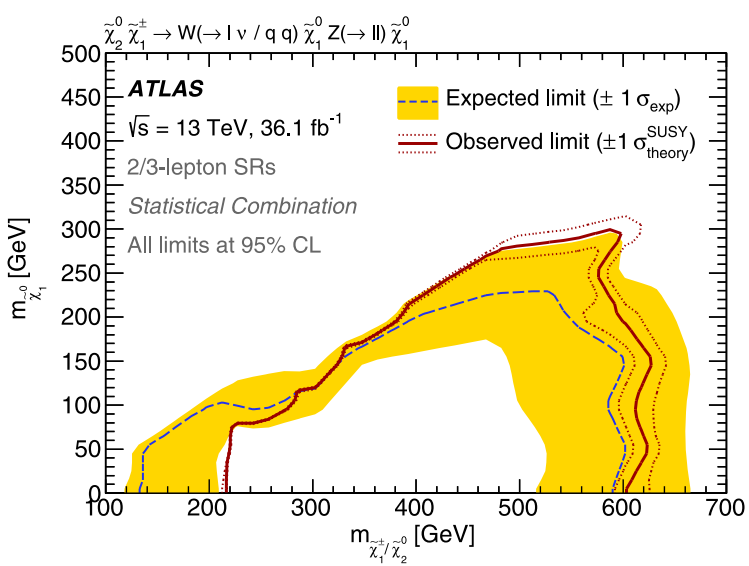

(c)

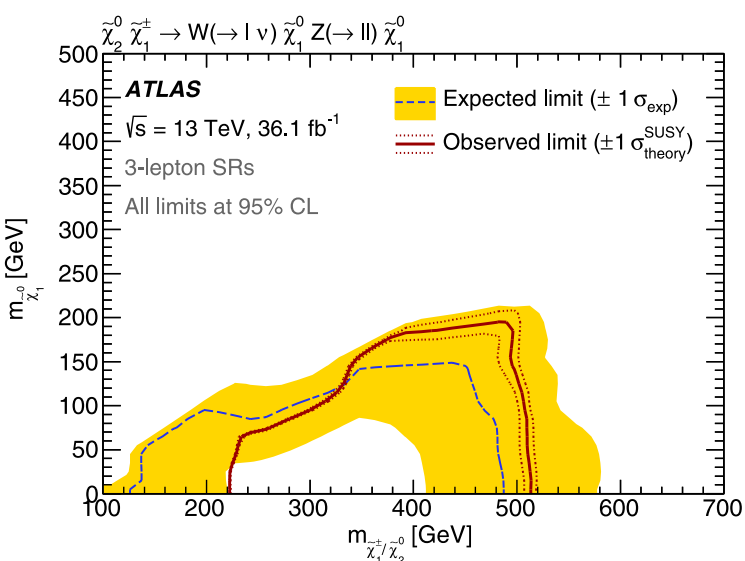

(b)

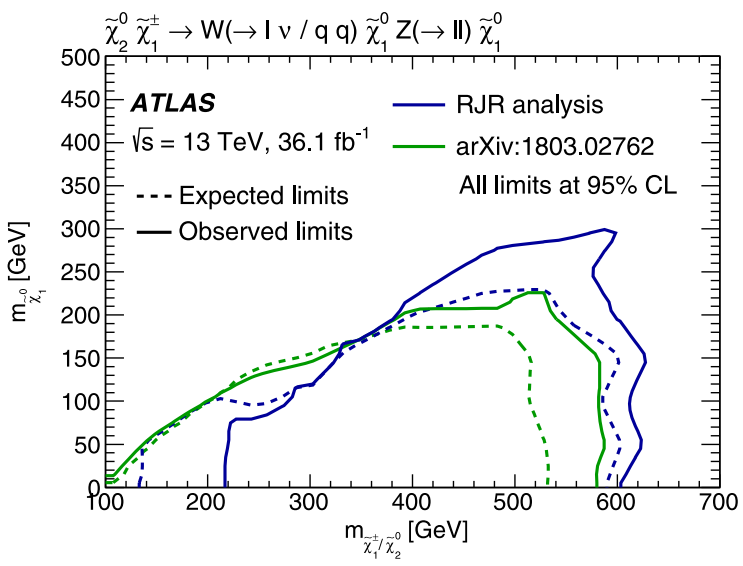

(d)

FIG. 13. Exclusion limits at $95 \%$ C.L. on the masses of $\tilde{\chi}_{1}^{ \pm} / \tilde{\chi}_{2}^{0}$ and $\tilde{\chi}_{1}^{0}$ from the analysis of $36.1 \mathrm{fb}^{-1}$ of $13 \mathrm{TeV} p p$ collision data obtained from the (a) $2 \ell$ search, (b) the $3 \ell$ search, (c) the statistical combination of the $2 \ell$ and $3 \ell$ search channels, assuming $100 \%$ branching ratio of the sparticles to decay to SM $W / Z$ bosons and $\tilde{\chi}_{1}^{0}$. The dashed line and the shaded band are the expected limit and its $\pm 1 \sigma$ uncertainty, respectively. The thick solid line is the observed limit for the central value of the signal cross section. The dotted lines around the observed limit illustrate the change in the observed limit as the nominal signal cross section is scaled up and down by the theoretical uncertainty and (d) comparison between the exclusion limits from this analysis and Ref. [18].

the data. A comparison with the analysis from Ref. [18] in Fig. 13(d) shows that there is a region of phase space in this simplified model, excluded at $95 \%$ C.L. by that analysis, that cannot be excluded by the results of this analysis.

The RJR selection reduces background through testing how well the events exhibit properties anticipated for the topologies under investigation with a much looser requirement on the missing transverse momentum than in the analysis in Ref. [18]. The methods by which the analyses select the putative $Z$-boson candidate and define SRs with or without a system of jets consistent with ISR also differ. The overlap of the selected data events in the SRs between the two approaches is found to be smaller than $20 \%$ and $30 \%$ for the two-lepton and three-lepton channels, respectively. In the compressed regions the overlap percentage for the hypothetical signal $m_{\tilde{\chi}_{1}^{ \pm} / \tilde{\chi}_{2}^{0}}=200 \mathrm{GeV}$ and $m_{\tilde{\chi}_{1}^{0}}=$ $100 \mathrm{GeV}$ for the $2 \ell(3 \ell)$ search channel is found to be less than $5 \%(15 \%)$.
In light of these results in the SR3 $\ell$ ISR, SR3 $\ell$ LLow, $\mathrm{SR} 2 \ell$ ISR and SR $2 \ell$ Low regions, a variety of crosschecks were performed for both the $2 \ell$ and $3 \ell$ channels.

TABLE XVIII. Breakdown of the observed and expected (in parentheses) number of events in terms of flavor composition in the SRs with an excess.

\begin{tabular}{lcc}
\hline \hline Signal region & SR2 $\ell$ Low & SR2 $\ell$ _ISR \\
\hline$e e$ & $9(4.5 \pm 3.9)$ & $3(1.2 \pm 1.2)$ \\
$\mu \mu$ & $10(3.9 \pm 2.6)$ & $8(1.5 \pm 1.5)$ \\
Signal region & SR3 $\ell$ Low & SR3 $\ell$ _ISR \\
$e е e$ & $6(3.5 \pm 0.7)$ & $3(1.1 \pm 0.3)$ \\
$e е \mu$ & $6(2.0 \pm 0.4)$ & $3(0.9 \pm 0.3)$ \\
$\mu \mu \mu$ & $7(2.7 \pm 0.6)$ & $4(1.5 \pm 0.4)$ \\
$\mu \mu e$ & $1(1.9 \pm 0.4)$ & $2(0.4 \pm 0.1)$ \\
\hline
\end{tabular}


Table XVIII shows the breakdown of the composition of the lepton flavor for the events selected in the SR2 $\ell$ Low, $\mathrm{SR} 2 \ell$ ISR, SR $3 \ell$ Low and SR3 $\ell$ ISR regions, along with the expectation from the background estimation.

The validation-region distributions in Figs. 5 and 7 show that there is good agreement between the expectation from the background prediction and data in kinematic regions close to the SRs. For the SR $3 \ell_{\text {IISR }}$ and SR $3 \ell_{\text {LL Low }}$ regions, where the excesses are most significant, the composition of the events is studied in dedicated validation regions where the primary selection criteria in the signal region are inverted. In each of these distributions the observed events are in good agreement with the prediction, and the primary background from $W Z$ events in $\mathrm{MC}$ simulation describes the data in both shape and yield. These cross-checks do not indicate a significant mismodeling of any single component of the background. In all cases the main background components are studied with alternative generators and there is good agreement between these samples. Yields of events determined with datadriven methods are cross-checked with MC simulation samples and no significant discrepancies are observed.

\section{CONCLUSION}

The paper presents a search for the electroweak production of neutralinos and charginos decaying into final states with exactly two or three electrons or muons and missing transverse momentum, performed using protonproton collision data at $\sqrt{s}=13 \mathrm{TeV}$ corresponding to an integrated luminosity of $36.1 \mathrm{fb}^{-1}$ recorded by the ATLAS detector at the LHC. Two distinct search channels based on recursive jigsaw reconstruction are considered where both the $2 \ell$ and $3 \ell$ channels target the same signal mode but with the $W$ boson decaying leptonically or to jets.

The statistical interpretation of the two search channels places exclusion limits on associated $\tilde{\chi}_{1}^{ \pm} \tilde{\chi}_{2}^{0}$ production with gauge-boson-mediated decays. For a massless $\tilde{\chi}_{1}^{0}, \tilde{\chi}_{1}^{ \pm} / \tilde{\chi}_{2}^{0}$ masses up to $600 \mathrm{GeV}$ are excluded. The results extend the region of supersymmetric parameter space previously excluded by LHC searches in the high- and intermediate-mass regions. In the low-mass and ISR signal regions an excess of events above the SM prediction is observed and the region of parameter space below $m_{\tilde{\chi}_{1}^{ \pm} / \tilde{\chi}_{2}^{0}}=$ $220 \mathrm{GeV}$ cannot be excluded.

The excesses observed in the $2 \ell$ and $3 \ell$ channels in the ISR (low-mass) signal regions correspond to local significances of 2.0 and 3.0 (1.4 and 2.1) standard deviations, respectively.

\section{ACKNOWLEDGMENTS}

We thank CERN for the very successful operation of the LHC, as well as the support staff from our institutions without whom ATLAS could not be operated efficiently. We acknowledge the support of ANPCyT, Argentina; YerPhI, Armenia; ARC, Australia; BMWFW and FWF, Austria; ANAS, Azerbaijan; SSTC, Belarus; CNPq and FAPESP, Brazil; NSERC, NRC and CFI, Canada; CERN; CONICYT, Chile; CAS, MOST and NSFC, China; COLCIENCIAS, Colombia; MSMT CR, MPO CR and VSC CR, Czech Republic; DNRF and DNSRC, Denmark; IN2P3-CNRS, CEA-DRF/IRFU, France; SRNSFG, Georgia; BMBF, HGF, and MPG, Germany; GSRT, Greece; RGC, Hong Kong SAR, China; ISF, ICORE and Benoziyo Center, Israel; INFN, Italy; MEXT and JSPS, Japan; CNRST, Morocco; NWO, Netherlands; RCN, Norway; MNiSW and NCN, Poland; FCT, Portugal; MNE/IFA, Romania; MES of Russia and NRC KI, Russian Federation; JINR; MESTD, Serbia; MSSR, Slovakia; ARRS and MIZŠ, Slovenia; DST/NRF, South Africa; MINECO, Spain; SRC and Wallenberg Foundation, Sweden; SERI, SNSF and Cantons of Bern and Geneva, Switzerland; MOST, Taiwan; TAEK, Turkey; STFC, United Kingdom; DOE and NSF, United States of America. In addition, individual groups and members have received support from BCKDF, the Canada Council, CANARIE, CRC, Compute Canada, FQRNT, and the Ontario Innovation Trust, Canada; EPLANET, ERC, ERDF, FP7, Horizon 2020 and Marie Skłodowska-Curie Actions, European Union; Investissements d'Avenir Labex and Idex, ANR, Région Auvergne and Fondation Partager le Savoir, France; DFG and AvH Foundation, Germany; Herakleitos, Thales and Aristeia programmes co-financed by EU-ESF and the Greek NSRF; BSF, GIF and Minerva, Israel; BRF, Norway; CERCA Programme Generalitat de Catalunya, Generalitat Valenciana, Spain; the Royal Society and Leverhulme Trust, United Kingdom. The crucial computing support from all WLCG partners is acknowledged gratefully, in particular from CERN, the ATLAS Tier-1 facilities at TRIUMF (Canada), NDGF (Denmark, Norway, Sweden), CC-IN2P3 (France), KIT/GridKA (Germany), INFN-CNAF (Italy), NL-T1 (Netherlands), PIC (Spain), ASGC (Taiwan), RAL (UK) and BNL (USA), the Tier-2 facilities worldwide and large non-WLCG resource providers. Major contributors of computing resources are listed in Ref. [100]. 
[1] Y. A. Golfand and E. P. Likhtman, Pis'ma Zh. Eksp. Teor. Fiz. 13, 452 (1971) [Extension of the algebra of Poincare group generators and violation of $\mathrm{p}$ invariance, JETP Lett. 13, 323 (1971)].

[2] D. V. Volkov and V. P. Akulov, Is the neutrino a goldstone particle?, Phys. Lett. 46B, 109 (1973).

[3] J. Wess and B. Zumino, Supergauge transformations in four-dimensions, Nucl. Phys. B70, 39 (1974).

[4] J. Wess and B. Zumino, Supergauge invariant extension of quantum electrodynamics, Nucl. Phys. B78, 1 (1974).

[5] S. Ferrara and B. Zumino, Supergauge invariant YangMills theories, Nucl. Phys. B79, 413 (1974).

[6] A. Salam and J. A. Strathdee, Super-symmetry and nonAbelian gauges, Phys. Lett. 51B, 353 (1974).

[7] G. R. Farrar and P. Fayet, Phenomenology of the production, decay, and detection of new hadronic states associated with supersymmetry, Phys. Lett. 76B, 575 (1978).

[8] H. Goldberg, Constraint on the Photino Mass from Cosmology, Phys. Rev. Lett. 50, 1419 (1983); Erratum 103, 099905 (2009).

[9] J. R. Ellis, J. S. Hagelin, D. V. Nanopoulos, K. A. Olive, and M. Srednicki, Supersymmetric relics from the big bang, Nucl. Phys. B238, 453 (1984).

[10] ATLAS Collaboration, Search for squarks and gluinos in final states with jets and missing transverse momentum using $36 \mathrm{fb}^{-1}$ of $\sqrt{s}=13 \mathrm{TeV} p p$ collision data with the ATLAS detector, Phys. Rev. D 97, 112001 (2018).

[11] CMS Collaboration, Search for supersymmetry in multijet events with missing transverse momentum in protonproton collisions at $13 \mathrm{TeV}$, Phys. Rev. D 96, 032003 (2017).

[12] CMS Collaboration, Search for new phenomena with the $M_{\mathrm{T} 2}$ variable in the all-hadronic final state produced in proton-proton collisions at $\sqrt{s}=13 \mathrm{TeV}$, Eur. Phys. J. C 77, 710 (2017).

[13] P. Jackson, C. Rogan, and M. Santoni, Sparticles in motion: Analyzing compressed SUSY scenarios with a new method of event reconstruction, Phys. Rev. D 95, 035031 (2017).

[14] P. Jackson and C. Rogan, Recursive Jigsaw reconstruction: HEP event analysis in the presence of kinematic and combinatoric ambiguities, Phys. Rev. D 96, 112007 (2017).

[15] ATLAS Collaboration, Search for direct production of charginos, neutralinos and sleptons in final states with two leptons and missing transverse momentum in $p p$ collisions at $\sqrt{s}=8 \mathrm{TeV}$ with the ATLAS detector, J. High Energy Phys. 05 (2014) 071.

[16] ATLAS Collaboration, Search for direct production of charginos and neutralinos in events with three leptons and missing transverse momentum in $\sqrt{s}=8 \mathrm{TeV} p p$ collisions with the ATLAS detector, J. High Energy Phys. 04 (2014) 169

[17] ATLAS Collaboration, Search for the electroweak production of supersymmetric particles in $\sqrt{s}=8 \mathrm{TeV} p p$ collisions with the ATLAS detector, Phys. Rev. D 93, 052002 (2016).

[18] ATLAS Collaboration, Search for electroweak production of supersymmetric particles in final states with two or three leptons at $\sqrt{s}=13 \mathrm{TeV}$ with the ATLAS detector, arXiv:1803.02762.

[19] CMS Collaboration, Search for electroweak production of charginos and neutralinos in multilepton final states in proton-proton collisions at $\sqrt{s}=13 \mathrm{TeV}$, J. High Energy Phys. 03 (2018) 166.

[20] CMS Collaboration, Search for new phenomena in final states with two opposite-charge, same-flavor leptons, jets, and missing transverse momentum in $\mathrm{pp}$ collisions at $\sqrt{s}=13 \mathrm{TeV}$, J. High Energy Phys. 03 (2018) 076.

[21] CMS Collaboration, Combined search for electroweak production of charginos and neutralinos in proton-proton collisions at $\sqrt{s}=13 \mathrm{TeV}$, J. High Energy Phys. 03 (2018) 160.

[22] ATLAS Collaboration, The ATLAS experiment at the CERN large hadron collider, J. Instrum. 3, S08003 (2008).

[23] ATLAS Collaboration, ATLAS insertable B-layer technical design report, CERN Report No. ATLAS-TDR-19ADD-1, 2010, https://cdsweb.cern.ch/record/1291633.

[24] ATLAS Collaboration, Performance of the ATLAS trigger system in 2015, Eur. Phys. J. C 77, 317 (2017).

[25] ATLAS Collaboration, Luminosity determination in pp collisions at $\sqrt{s}=8 \mathrm{TeV}$ using the ATLAS detector at the LHC, Eur. Phys. J. C 76, 653 (2016).

[26] ATLAS Collaboration, Monte Carlo generators for the production of a $W$ or $Z / \gamma^{*}$ Boson in association with jets at ATLAS in run 2, CERN Report No. ATL-PHYS-PUB2016-003, 2016, https://cds.cern.ch/record/2120133.

[27] T. Gleisberg, S. Höche, F. Krauss, M. Schönherr, S. Schumann, F. Siegert, and J. Winter, Event generation with SHERPA 1.1, J. High Energy Phys. 02 (2009) 007.

[28] R. D. Ball et al., Parton distributions for the LHC Run II, J. High Energy Phys. 04 (2015) 040.

[29] T. Gleisberg and S. Höche, Comix, a new matrix element generator, J. High Energy Phys. 12 (2008) 039.

[30] F. Cascioli, P. Maierhöfer, and S. Pozzorini, Scattering Amplitudes with OPEN LOOPS, Phys. Rev. Lett. 108, 111601 (2012).

[31] S. Schumann and F. Krauss, A Parton shower algorithm based on Catani-Seymour dipole factorisation, J. High Energy Phys. 03 (2008) 038.

[32] S. Höche, F. Krauss, M. Schönherr, and F. Siegert, QCD matrix elements + parton showers: The NLO case, J. High Energy Phys. 04 (2013) 027.

[33] H.-L. Lai, M. Guzzi, J. Huston, Z. Li, P. M. Nadolsky, J. Pumplin, and C.-P. Yuan, New parton distributions for collider physics, Phys. Rev. D 82, 074024 (2010).

[34] S. Alioli, P. Nason, C. Oleari, and E. Re, A general framework for implementing NLO calculations in shower Monte Carlo programs: the POWHEG BOX, J. High Energy Phys. 06 (2010) 043.

[35] ATLAS Collaboration, Simulation of top quark production for the ATLAS experiment at $\sqrt{s}=13 \mathrm{TeV}, \mathrm{CERN}$ Report No. ATL-PHYS-PUB-2016-004, 2016, https:// cds.cern.ch/record/2120417.

[36] S. Frixione, P. Nason, and C. Oleari, Matching NLO QCD computations with parton shower simulations: The POWHEG method, J. High Energy Phys. 11 (2007) 070.

[37] P. Artoisenet, R. Frederix, O. Mattelaer, and R. Rietkerk, Automatic spin-entangled decays of heavy resonances in 
Monte Carlo simulations, J. High Energy Phys. 03 (2013) 015.

[38] T. Sjöstrand, S. Mrenna, and P. Skands, PythiA 6.4 physics and manual, J. High Energy Phys. 05 (2006) 026.

[39] J. Pumplin, D. R. Stump, J. Huston, H.-L. Lai, P. Nadolsky, and W.-K. Tung, New generation of parton distributions with uncertainties from global QCD analysis, J. High Energy Phys. 07 (2002) 012.

[40] P. Z. Skands, Tuning Monte Carlo generators: The Perugia tunes, Phys. Rev. D 82, 074018 (2010).

[41] M. Czakon, P. Fiedler, and A. Mitov, Total Top-Quark PairProduction Cross Section at Hadron Colliders through $O\left(\alpha_{\mathrm{S}}^{4}\right)$, Phys. Rev. Lett. 110, 252004 (2013).

[42] M. Czakon and A. Mitov, Top++: A program for the calculation of the top-pair cross-section at hadron colliders, Comput. Phys. Commun. 185, 2930 (2014).

[43] N. Kidonakis, Two-loop soft anomalous dimensions for single top quark associated production with a $W^{-}$or $H^{-}$, Phys. Rev. D 82, 054018 (2010).

[44] N. Kidonakis, Next-to-next-to-leading-order collinear and soft gluon corrections for t-channel single top quark production, Phys. Rev. D 83, 091503 (2011).

[45] M. Aliev, H. Lacker, U. Langenfeld, S. Moch, P. Uwer, and M. Wiedermann, HATHOR: HAdronic top and Heavy quarks crOss section calculatoR, Comput. Phys. Commun. 182, 1034 (2011).

[46] P. Kant, O. M. Kind, T. Kintscher, T. Lohse, T. Martini, S. Mölbitz, P. Rieck, and P. Uwer, HatHor for single topquark production: Updated predictions and uncertainty estimates for single top-quark production in hadronic collisions, Comput. Phys. Commun. 191, 74 (2015).

[47] J. Alwall, R. Frederix, S. Frixione, V. Hirschi, F. Maltoni, O. Mattelaer, H.-S. Shao, T. Stelzer, P. Torrielli, and M. Zaro, The automated computation of tree-level and nextto-leading order differential cross sections, and their matching to parton shower simulations, J. High Energy Phys. 07 (2014) 079.

[48] T. Sjöstrand, S. Ask, J. R. Christiansen, R. Corke, N. Desai, P. Ilten, S. Mrenna, S. Prestel, C. O. Rasmussen, and P.Z. Skands, An introduction to PYTHIA 8.2, Comput. Phys. Commun. 191, 159 (2015).

[49] ATLAS Collaboration, Modelling of the $t \bar{t} H$ and $t \bar{t} V$ $(V=W, Z$ ) processes for $\sqrt{s}=13 \mathrm{TeV}$ ATLAS analyses, CERN Report No. ATL-PHYS-PUB-2016-005, 2016, https://cds.cern.ch/record/2120826.

[50] A. Lazopoulos, T. McElmurry, K. Melnikov, and F. Petriello, Next-to-leading order QCD corrections to $t \bar{t} Z$ production at the LHC, Phys. Lett. B 666, 62 (2008).

[51] J. M. Campbell and R. K. Ellis, $t \bar{t} W^{ \pm}$production and decay at NLO, J. High Energy Phys. 07 (2012) 052.

[52] ATLAS Collaboration, Multi-Boson simulation for $13 \mathrm{TeV}$ ATLAS analyses, CERN Report No. ATL-PHYS-PUB2016-002, 2016, https://cds.cern.ch/record/2119986.

[53] J. M. Campbell and R. K. Ellis, An update on vector boson pair production at hadron colliders, Phys. Rev. D 60, 113006 (1999).

[54] J. M. Campbell, R. K. Ellis, and C. Williams, Vector boson pair production at the LHC, J. High Energy Phys. 07 (2011) 018.
[55] S. Schumann and F. Krauss, A Parton shower algorithm based on Catani-Seymour dipole factorisation, J. High Energy Phys. 03 (2008) 038.

[56] S. Höche, F. Krauss, S. Schumann, and F. Siegert, QCD matrix elements and truncated showers, J. High Energy Phys. 05 (2009) 053.

[57] ATLAS Collaboration, Multi-Boson simulation for $13 \mathrm{TeV}$ ATLAS analyses, CERN Report No. ATL-PHYS-PUB2017-005, 2017, https://cds.cern.ch/record/2261933.

[58] G. Corcella, I. G. Knowles, G. Marchesini, S. Moretti, K. Odagiri, P. Richardson, M. H. Seymour, and B. R. Webber, HERWIG 6.5: An event generator for hadron emission reactions with interfering gluons (including supersymmetric processes), J. High Energy Phys. 01 (2001) 010.

[59] S. Dittmaier et al., Handbook of LHC Higgs cross sections: 2. differential distributions, CERN Report No. CERN2012-002, arXiv:1201.3084, DOI: 10.5170/CERN-2012002.

[60] J. Alwall, P. C. Schuster, and N. Toro, Simplified models for a first characterization of new physics at the LHC, Phys. Rev. D 79, 075020 (2009).

[61] F. Maltoni and T. Stelzer, MadEvent: Automatic event generation with MADGRAPH, J. High Energy Phys. 02 (2003) 027.

[62] ATLAS Collaboration, ATLAS Pythia 8 tunes to $7 \mathrm{TeV}$ data, CERN Report No. ATL-PHYS-PUB-2014-021, 2014, https://cds.cern.ch/record/1966419.

[63] L. Lönnblad and S. Prestel, Matching tree-level matrix elements with interleaved showers, J. High Energy Phys. 03 (2012) 019.

[64] W. Beenakker, R. Höpker, M. Spira, and P. M. Zerwas, Squark and gluino production at hadron colliders, Nucl. Phys. B492, 51 (1997).

[65] A. Kulesza and L. Motyka, Threshold Resummation for Squark-Antisquark and Gluino-Pair Production at the LHC, Phys. Rev. Lett. 102, 111802 (2009).

[66] A. Kulesza and L. Motyka, Soft gluon resummation for the production of gluino-gluino and squark-antisquark pairs at the LHC, Phys. Rev. D 80, 095004 (2009).

[67] W. Beenakker, S. Brensing, M. Krämer, A. Kulesza, E. Laenen, and I. Niessen, Soft-gluon resummation for squark and gluino hadroproduction, J. High Energy Phys. 12 (2009) 041.

[68] W. Beenakker, SILJA BRENSING, M. Krämer, A. Kulesza, E. Laenen, L. Motyka, and I. Niessen, Squark and gluino hadroproduction, Int. J. Mod. Phys. A 26, 2637 (2011).

[69] C. Borschensky, M. Krämer, A. Kulesza, M. Mangano, S. Padhi, T. Plehn, and X. Portell, Squark and gluino production cross sections in $\mathrm{pp}$ collisions at $\sqrt{\mathrm{s}}=13$, 14, 33 and 100 TeV, Eur. Phys. J. C 74, 3174 (2014).

[70] D. J. Lange, The EVTGEN particle decay simulation package, Nucl. Instrum. Methods Phys. Res., Sect. A 462, 152 (2001).

[71] ATLAS Collaboration, Summary of ATLAS PYTHIA 8 tunes, CERN Report No. ATL-PHYS-PUB-2012-003, 2012, https://cdsweb.cern.ch/record/1474107.

[72] A. D. Martin, W. J. Stirling, R. S. Thorne, and G. Watt, Parton distributions for the LHC, Eur. Phys. J. C 63, 189 (2009). 
[73] ATLAS Collaboration, The ATLAS simulation infrastructure, Eur. Phys. J. C 70, 823 (2010).

[74] S. Agostinelli et al., GEANT4: A simulation toolkit, Nucl. Instrum. Methods Phys. Res., Sect. A 506, 250 (2003).

[75] ATLAS Collaboration, Muon reconstruction performance of the ATLAS detector in proton-proton collision data at $\sqrt{s}=13 \mathrm{TeV}$, Eur. Phys. J. C 76, 292 (2016).

[76] ATLAS Collaboration, Electron efficiency measurements with the ATLAS detector using the 2015 LHC protonproton collision data, CERN Report No. ATLAS-CONF2016-024, 2016, http://cds.cern.ch/record/2157687.

[77] M. Cacciari, G. P. Salam, and G. Soyez, The anti- $k_{t}$ jet clustering algorithm, J. High Energy Phys. 04 (2008) 063.

[78] M. Cacciari and G. P. Salam, Dispelling the $N^{3}$ myth for the $k_{t}$ jet-finder, Phys. Lett. B 641, 57 (2006).

[79] M. Cacciari, G. P. Salam, and G. Soyez, FastJet user manual, Eur. Phys. J. C 72, 1896 (2012).

[80] ATLAS Collaboration, Topological cell clustering in the ATLAS calorimeters and its performance in LHC Run 1, Eur. Phys. J. C 77, 490 (2017).

[81] M. Cacciari and G. P. Salam, Pileup subtraction using jet areas, Phys. Lett. B 659, 119 (2008).

[82] ATLAS Collaboration, Performance of pile-up mitigation techniques for jets in $p p$ collisions at $\sqrt{s}=8 \mathrm{TeV}$ using the ATLAS detector, Eur. Phys. J. C 76, 581 (2016).

[83] ATLAS Collaboration, Jet energy scale measurements and their systematic uncertainties in proton-proton collisions at $\sqrt{s}=13 \mathrm{TeV}$ with the ATLAS detector, Phys. Rev. D 96, 072002 (2017).

[84] ATLAS Collaboration, Tagging and suppression of pileup jets with the ATLAS detector, CERN Report No. ATLAS-CONF-2014-018, 2014, https://cds.cern.ch/ record/1700870.

[85] ATLAS Collaboration, Performance of $b$-Jet Identification in the ATLAS Experiment, J. Instrum. 11, P04008 (2016).

[86] ATLAS Collaboration, Optimisation of the ATLAS btagging performance for the 2016 LHC Run, CERN Report No. ATL-PHYS-PUB-2016-012, 2016, https://cds.cern.ch/ record/2160731.

[87] ATLAS Collaboration, Measurement of the photon identification efficiencies with the ATLAS detector using LHC Run-1 data, Eur. Phys. J. C 76, 666 (2016).

[88] M. Aaboud et al., Performance of missing transverse momentum reconstruction with the ATLAS detector using proton-proton collisions at $\sqrt{s}=13 \mathrm{TeV}$, arXiv:1802 .08168 .
[89] M. Baak, G. J. Besjes, D. Côté, A. Koutsman, J. Lorenz, and D. Short, HistFitter software framework for statistical data analysis, Eur. Phys. J. C 75, 153 (2015).

[90] A. L. Read, Presentation of search results: The $\mathrm{CL}_{\mathrm{s}}$ technique, J. Phys. G 28, 2693 (2002).

[91] M. Aaboud et al., Search for a scalar partner of the top quark in the jets plus missing transverse momentum final state at $\sqrt{s}=13 \mathrm{TeV}$ with the ATLAS detector, J. High Energy Phys. 12 (2017) 085.

[92] M. Aaboud et al., Search for top-squark pair production in final states with one lepton, jets, and missing transverse momentum using $36 \mathrm{fb}^{-1}$ of $\sqrt{s}=13 \mathrm{TeV} p p$ collision data with the ATLAS detector, J. High Energy Phys. 06 (2018) 108.

[93] ATLAS Collaboration, Measurement of the top quark-pair production cross section with ATLAS in $p p$ collisions at $\sqrt{s}=7 \mathrm{TeV}$, Eur. Phys. J. C 71, 1577 (2011).

[94] ATLAS Collaboration, Search for new phenomena in events containing a same-flavour opposite-sign dilepton pair, jets, and large missing transverse momentum in $\sqrt{s}=$ $13 \mathrm{TeV} p p$ collisions with the ATLAS detector, Eur. Phys. J. C 77, 144 (2017).

[95] CMS Collaboration, Search for physics beyond the standard model in events with a $Z$ boson, jets, and missing transverse energy in $p p$ collisions at $\sqrt{s}=7 \mathrm{TeV}$, Phys. Lett. B 716, 260 (2012).

[96] CMS Collaboration, Search for physics beyond the standard model in events with two leptons, jets, and missing transverse momentum in $p p$ collisions at $\sqrt{s}=8 \mathrm{TeV}$, J. High Energy Phys. 04 (2015) 124.

[97] ATLAS Collaboration, Expected performance of missing transverse momentum reconstruction for the ATLAS detector at $\sqrt{s}=13 \mathrm{TeV}$, CERN Report No. ATLPHYS-PUB-2015-023, 2015, https://cds.cern.ch/record/ 2037700.

[98] ATLAS Collaboration, Calibration of $b$-tagging using dileptonic top pair events in a combinatorial likelihood approach with the ATLAS experiment, CERN Report No. ATLAS-CONF-2014-004, 2014, https://cds.cern.ch/ record/1664335.

[99] ATLAS Collaboration, Calibration of the performance of $b$-tagging for $c$ and light-flavour jets in the 2012 ATLAS data, CERN Report No. ATLAS-CONF-2014-046, 2014, https://cds.cern.ch/record/1741020.

[100] ATLAS Collaboration, ATLAS Computing Acknowledgements 2016-2017, CERN Report No. ATL-GEN-PUB2016-002, 2016, https://cds.cern.ch/record/2202407.

M. Aaboud ${ }^{34 d}$ G. Aad,${ }^{99}$ B. Abbott, ${ }^{125}$ O. Abdinov, ${ }^{13, a}$ B. Abeloos, ${ }^{129}$ D. K. Abhayasinghe, ${ }^{91}$ S. H. Abidi, ${ }^{164}$ O. S. AbouZeid, ${ }^{143}$ N. L. Abraham, ${ }^{153}$ H. Abramowicz,${ }^{158}$ H. Abreu, ${ }^{157}$ Y. Abulaiti, ${ }^{6}$ B. S. Acharya, ${ }^{64 a, 64 b, b}$ S. Adachi, ${ }^{160}$ L. Adamczyk, ${ }^{81 a}$ J. Adelman, ${ }^{119}$ M. Adersberger,${ }^{12}$ A. Adiguzel, ${ }^{12 c, c}$ T. Adye, ${ }^{141}$ A. A. Affolder, ${ }^{143}$ Y. Afik, ${ }^{157}$ C. Agheorghiesei, ${ }^{27 \mathrm{c}}$ J. A. Aguilar-Saavedra, ${ }^{137 f, 137 \mathrm{a}}$ F. Ahmadov, ${ }^{77, \mathrm{~d}}$ G. Aielli, ${ }^{71 \mathrm{a}, 71 \mathrm{~b}}$ S. Akatsuka, ${ }^{83}$ T. P. A. Åkesson, ${ }^{94}$ E. Akilli, ${ }^{52}$ A. V. Akimov, ${ }^{108}$ G. L. Alberghi, ${ }^{23 b, 23 a}$ J. Albert ${ }^{173}$ P. Albicocco,${ }^{49}$ M. J. Alconada Verzini,${ }^{86}$

S. Alderweireldt, ${ }^{117}$ M. Aleksa, ${ }^{35}$ I. N. Aleksandrov,${ }^{77}$ C. Alexa, ${ }^{27 b}$ G. Alexander, ${ }^{158}$ T. Alexopoulos, ${ }^{10}$ M. Alhroob, ${ }^{125}$ B. Ali ${ }^{139}$ G. Alimonti, ${ }^{66 a}$ J. Alison, ${ }^{36}$ S. P. Alkire, ${ }^{145}$ C. Allaire, ${ }^{129}$ B. M. M. Allbrooke, ${ }^{153}$ B. W. Allen, ${ }^{128}$ P. P. Allport, ${ }^{21}$ 
A. Aloisio, ${ }^{67 a, 67 b}$ A. Alonso, ${ }^{39}$ F. Alonso, ${ }^{86}$ C. Alpigiani, ${ }^{145}$ A. A. Alshehri, ${ }^{55}$ M. I. Alstaty, ${ }^{99}$ B. Alvarez Gonzalez, ${ }^{35}$ D. Álvarez Piqueras, ${ }^{171}$ M. G. Alviggi, ${ }^{67 a, 67 b}$ B. T. Amadio, ${ }^{18}$ Y. Amaral Coutinho, ${ }^{78 b}$ L. Ambroz, ${ }^{132}$ C. Amelung, ${ }^{26}$ D. Amidei, ${ }^{103}$ S. P. Amor Dos Santos, ${ }^{137 a, 137 c}$ S. Amoroso, ${ }^{35}$ C. S. Amrouche, ${ }^{52}$ C. Anastopoulos, ${ }^{146}$ L. S. Ancu, ${ }^{52}$ N. Andari, ${ }^{21}$ T. Andeen, ${ }^{11}$ C. F. Anders, ${ }^{59 b}$ J. K. Anders, ${ }^{20}$ K. J. Anderson, ${ }^{36}$ A. Andreazza, ${ }^{66 a, 66 b}$ V. Andrei, ${ }^{59 a}$ C. R. Anelli, ${ }^{173}$ S. Angelidakis, ${ }^{37}$ I. Angelozzi, ${ }^{118}$ A. Angerami, ${ }^{38}$ A. V. Anisenkov, ${ }^{120 b, 120 a}$ A. Annovi, ${ }^{69 a}$ C. Antel, ${ }^{59 a}$ M. T. Anthony, ${ }^{146}$ M. Antonelli, ${ }^{49}$ D. J. A. Antrim, ${ }^{168}$ F. Anulli, ${ }^{70 a}$ M. Aoki, ${ }^{79}$ L. Aperio Bella, ${ }^{35}$ G. Arabidze, ${ }^{104}$ Y. Arai, ${ }^{79}$ J. P. Araque, ${ }^{137 \mathrm{a}}$ V. Araujo Ferraz, ${ }^{78 b}$ R. Araujo Pereira, ${ }^{78 b}$ A. T. H. Arce, ${ }^{47}$ R. E. Ardell, ${ }^{91}$ F. A. Arduh, ${ }^{86}$ J-F. Arguin, ${ }^{107}$ S. Argyropoulos, ${ }^{75}$ A. J. Armbruster, ${ }^{35}$ L. J. Armitage, ${ }^{90}$ A. Armstrong, ${ }^{168}$ O. Arnaez, ${ }^{164}$ H. Arnold, ${ }^{18}$ M. Arratia, ${ }^{31}$ O. Arslan, ${ }^{24}$ A. Artamonov, ${ }^{109, a}$ G. Artoni, ${ }^{132}$ S. Artz, ${ }^{97}$ S. Asai, ${ }^{160}$ N. Asbah, ${ }^{44}$ A. Ashkenazi, ${ }^{158}$ E. M. Asimakopoulou, ${ }^{169}$ L. Asquith, ${ }^{153}$ K. Assamagan, ${ }^{29}$ R. Astalos, ${ }^{28 a}$ R. J. Atkin, ${ }^{32 a}$ M. Atkinson, ${ }^{170}$ N. B. Atlay, ${ }^{148}$ K. Augsten, ${ }^{139}$ G. Avolio, ${ }^{35}$ R. Avramidou, ${ }^{58 a}$ B. Axen, ${ }^{18}$ M. K. Ayoub, ${ }^{15 a}$ G. Azuelos, ${ }^{107, e}$ A. E. Baas, ${ }^{59 a}$ M. J. Baca, ${ }^{21}$ H. Bachacou, ${ }^{142}$ K. Bachas, ${ }^{65 a, 65 b}$ M. Backes, ${ }^{132}$ P. Bagnaia, ${ }^{70 a, 70 b}$ M. Bahmani, ${ }^{82}$ H. Bahrasemani, ${ }^{149}$ A. J. Bailey, ${ }^{171}$ J. T. Baines, ${ }^{141}$ M. Bajic, ${ }^{39}$ C. Bakalis, ${ }^{10}$ O. K. Baker, ${ }^{180}$ P. J. Bakker, ${ }^{118}$ D. Bakshi Gupta, ${ }^{93}$ E. M. Baldin, ${ }^{120 b, 120 a}$ P. Balek, ${ }^{177}$ F. Balli, ${ }^{142}$ W. K. Balunas, ${ }^{134}$ J. Balz, ${ }^{97}$ E. Banas, ${ }^{82}$ A. Bandyopadhyay, ${ }^{24}$ S. Banerjee, ${ }^{178, f}$ A. A. E. Bannoura, ${ }^{179}$ L. Barak, ${ }^{158}$ W. M. Barbe, ${ }^{37}$ E. L. Barberio, ${ }^{102}$ D. Barberis, ${ }^{53 b, 53 a}$ M. Barbero, ${ }^{99}$ T. Barillari, ${ }^{113}$ M-S. Barisits, ${ }^{35}$ J. Barkeloo, ${ }^{128}$ T. Barklow, ${ }^{150}$ N. Barlow, ${ }^{31}$ R. Barnea, ${ }^{157}$ S. L. Barnes, ${ }^{58 \mathrm{c}}$ B. M. Barnett, ${ }^{141}$ R. M. Barnett, ${ }^{18}$ Z. Barnovska-Blenessy, ${ }^{58 \mathrm{a}}$ A. Baroncelli, ${ }^{72 \mathrm{a}}$ G. Barone, ${ }^{26}$ A. J. Barr, ${ }^{132}$ L. Barranco Navarro, ${ }^{171}$ F. Barreiro, ${ }^{96}$ J. Barreiro Guimarães da Costa, ${ }^{15 a}$ R. Bartoldus, ${ }^{150}$ A. E. Barton, ${ }^{87}$ P. Bartos, ${ }^{28 a}$ A. Basalaev, ${ }^{135}$ A. Bassalat, ${ }^{129}$ R. L. Bates, ${ }^{55}$ S. J. Batista, ${ }^{164}$ S. Batlamous, ${ }^{34 \mathrm{e}}$ J. R. Batley, ${ }^{31}$ M. Battaglia, ${ }^{143}$ M. Bauce, ${ }^{70 a, 70 b}$ F. Bauer, ${ }^{142}$ K. T. Bauer, ${ }^{168}$ H. S. Bawa, ${ }^{150, g}$ J. B. Beacham, ${ }^{123}$ M. D. Beattie, ${ }^{87}$ T. Beau, ${ }^{133}$ P. H. Beauchemin, ${ }^{167}$ P. Bechtle, ${ }^{24}$ H. C. Beck, ${ }^{51}$ H. P. Beck, ${ }^{20, h}$ K. Becker, ${ }^{50}$ M. Becker, ${ }^{97}$ C. Becot, ${ }^{44}$ A. Beddall, ${ }^{12 d}$ A. J. Beddall, ${ }^{12 a}$ V. A. Bednyakov, ${ }^{77}$ M. Bedognetti, ${ }^{118}$ C. P. Bee, ${ }^{152}$ T. A. Beermann, ${ }^{35}$ M. Begalli, ${ }^{78 b}$ M. Begel, ${ }^{29}$ A. Behera, ${ }^{152}$ J. K. Behr, ${ }^{44}$ A. S. Bell, ${ }^{92}$ G. Bella, ${ }^{158}$ L. Bellagamba, ${ }^{23 b}$ A. Bellerive, ${ }^{33}$ M. Bellomo, ${ }^{157}$ P. Bellos, ${ }^{9}$ K. Belotskiy, ${ }^{110}$ N. L. Belyaev, ${ }^{110}$ O. Benary, ${ }^{158, a}$ D. Benchekroun, ${ }^{34 a}$ M. Bender, ${ }^{12}$ N. Benekos, ${ }^{10}$ Y. Benhammou, ${ }^{158}$ E. Benhar Noccioli, ${ }^{180}$ J. Benitez, ${ }^{75}$ D. P. Benjamin, ${ }^{47}$ M. Benoit, ${ }^{52}$ J. R. Bensinger, ${ }^{26}$ S. Bentvelsen, ${ }^{118}$ L. Beresford, ${ }^{132}$ M. Beretta, ${ }^{49}$ D. Berge, ${ }^{44}$ E. Bergeaas Kuutmann, ${ }^{169}$ N. Berger, ${ }^{5}$ L. J. Bergsten, ${ }^{26}$ J. Beringer, ${ }^{18}$ S. Berlendis, ${ }^{7}$ N. R. Bernard, ${ }^{100}$ G. Bernardi, ${ }^{133}$ C. Bernius, ${ }^{150}$ F. U. Bernlochner, ${ }^{24}$ T. Berry, ${ }^{91}$ P. Berta, ${ }^{97}$ C. Bertella, ${ }^{15 a}$ G. Bertoli, ${ }^{43 a, 43 b}$ I. A. Bertram, ${ }^{87}$ G. J. Besjes, ${ }^{39}$

O. Bessidskaia Bylund, ${ }^{43 a, 43 b}$ M. Bessner, ${ }^{44}$ N. Besson, ${ }^{142}$ A. Bethani, ${ }^{98}$ S. Bethke, ${ }^{113}$ A. Betti, ${ }^{24}$ A. J. Bevan, ${ }^{90}$ J. Beyer, ${ }^{113}$ R. M. Bianchi, ${ }^{136}$ O. Biebel, ${ }^{112}$ D. Biedermann, ${ }^{19}$ R. Bielski, ${ }^{98}$ K. Bierwagen, ${ }^{97}$ N. V. Biesuz, ${ }^{69 a, 69 b}$ M. Biglietti, ${ }^{72 a}$ T. R. V. Billoud, ${ }^{107}$ M. Bindi, ${ }^{51}$ A. Bingul, ${ }^{12 d}$ C. Bini, ${ }^{70 a, 70 b}$ S. Biondi, ${ }^{23 b, 23 a}$ T. Bisanz, ${ }^{51}$ J. P. Biswal, ${ }^{158}$ C. Bittrich, ${ }^{46}$ D. M. Bjergaard, ${ }^{47}$ J. E. Black, ${ }^{150}$ K. M. Black, ${ }^{25}$ R. E. Blair, ${ }^{6}$ T. Blazek, ${ }^{28 a}$ I. Bloch, $^{44}$ C. Blocker, ${ }^{26}$ A. Blue, ${ }^{55}$ U. Blumenschein, ${ }^{90}$ Dr. Blunier, ${ }^{14 a}$ G. J. Bobbink, ${ }^{118}$ V. S. Bobrovnikov, ${ }^{120 b, 120 a}$ S. S. Bocchetta, ${ }^{94}$ A. Bocci, ${ }^{47}$ D. Boerner, ${ }^{179}$ D. Bogavac, ${ }^{112}$ A. G. Bogdanchikov, ${ }^{120 b, 120 a}$ C. Bohm, ${ }^{43 a}$ V. Boisvert, ${ }^{91}$ P. Bokan, ${ }^{169}$ T. Bold, ${ }^{81 a}$ A. S. Boldyrev, ${ }^{111}$ A. E. Bolz, ${ }^{59 b}$ M. Bomben, ${ }^{133}$ M. Bona, ${ }^{90}$ J. S. Bonilla, ${ }^{128}$ M. Boonekamp, ${ }^{142}$ A. Borisov, ${ }^{121}$ G. Borissov, ${ }^{87}$ J. Bortfeldt, ${ }^{35}$ D. Bortoletto, ${ }^{132}$ V. Bortolotto, ${ }^{71 a, 61 b, 61 c, 71 b}$ D. Boscherini, ${ }^{23 b}$ M. Bosman, ${ }^{14}$ J. D. Bossio Sola, ${ }^{30}$ K. Bouaouda, ${ }^{34 a}$ J. Boudreau, ${ }^{136}$ E. V. Bouhova-Thacker, ${ }^{87}$ D. Boumediene, ${ }^{37}$ C. Bourdarios, ${ }^{129}$ S. K. Boutle, ${ }^{55}$ A. Boveia, ${ }^{123}$ J. Boyd, ${ }^{35}$ I. R. Boyko, ${ }^{77}$ A. J. Bozson, ${ }^{91}$ J. Bracinik, $^{21}$ N. Brahimi, ${ }^{99}$ A. Brandt, ${ }^{8}$ G. Brandt, ${ }^{179}$ O. Brandt, ${ }^{59}$ F. Braren, ${ }^{44}$ U. Bratzler, ${ }^{161}$ B. Brau, ${ }^{100}$ J. E. Brau, ${ }^{128}$ W. D. Breaden Madden, ${ }^{55}$ K. Brendlinger, ${ }^{44}$ A. J. Brennan, ${ }^{102}$ L. Brenner, ${ }^{44}$ R. Brenner, ${ }^{169}$ S. Bressler, ${ }^{177}$ B. Brickwedde, ${ }^{97}$ D. L. Briglin, ${ }^{21}$ D. Britton, ${ }^{55}$ D. Britzger, ${ }^{59 b}$ I. Brock, $^{24}$ R. Brock, ${ }^{104}$ G. Brooijmans, ${ }^{38}$ T. Brooks, ${ }^{91}$ W. K. Brooks, ${ }^{144 b}$ E. Brost, ${ }^{119}$ J. H. Broughton, ${ }^{21}$ P. A. Bruckman de Renstrom, ${ }^{82}$ D. Bruncko, ${ }^{28 b}$ A. Bruni, ${ }^{23 b}$ G. Bruni, ${ }^{23 b}$ L. S. Bruni, ${ }^{118}$ S. Bruno, ${ }^{71 a, 71 b}$ B. H. Brunt, ${ }^{31}$ M. Bruschi, ${ }^{23 b}$ N. Bruscino, ${ }^{136}$ P. Bryant, ${ }^{36}$ L. Bryngemark, ${ }^{44}$ T. Buanes, ${ }^{17}$ Q. Buat, ${ }^{35}$ P. Buchholz, ${ }^{148}$ A. G. Buckley, ${ }^{55}$ I. A. Budagov, ${ }^{77}$ M. K. Bugge, ${ }^{131}$ F. Bührer, ${ }^{50}$ O. Bulekov, ${ }^{110}$ D. Bullock, ${ }^{8}$ T. J. Burch, ${ }^{119}$ S. Burdin, ${ }^{88}$ C. D. Burgard, ${ }^{118}$ A. M. Burger, B. Burghgrave, ${ }^{5}$ K. Burka, ${ }^{82}$ S. Burke, ${ }^{141}$ I. Burmeister, ${ }^{45}$ J. T. P. Burr, ${ }^{132}$ D. Büscher, ${ }^{50}$ V. Büscher, ${ }^{97}$ E. Buschmann, ${ }^{51}$ P. Bussey, ${ }^{55}$ J. M. Butler, ${ }^{25}$ C. M. Buttar, ${ }^{55}$ J. M. Butterworth, ${ }^{92}$ P. Butti, ${ }^{35}$ W. Buttinger, ${ }^{35}$ A. Buzatu, ${ }^{155}$ A. R. Buzykaev, ${ }^{120 b, 120 a}$ G. Cabras, ${ }^{23 b, 23 a}$ S. Cabrera Urbán, ${ }^{171}$ D. Caforio, ${ }^{139}$ H. Cai, ${ }^{170}$ V. M. M. Cairo, ${ }^{2}$ O. Cakir, ${ }^{4 a}$ N. Calace, ${ }^{52}$ P. Calafiura, ${ }^{18}$ A. Calandri, ${ }^{99}$ G. Calderini, ${ }^{133}$ P. Calfayan, ${ }^{63}$ G. Callea, ${ }^{40 b, 40 a}$ L. P. Caloba, ${ }^{78 b}$ S. Calvente Lopez, ${ }^{96}$ D. Calvet, ${ }^{37}$ S. Calvet, ${ }^{37}$ T. P. Calvet, ${ }^{152}$ M. Calvetti, ${ }^{69 a, 69 b}$ R. Camacho Toro, ${ }^{133}$ S. Camarda, ${ }^{35}$ P. Camarri, ${ }^{71 a, 71 b}$ D. Cameron, ${ }^{131}$ R. Caminal Armadans, ${ }^{100}$ C. Camincher, ${ }^{35}$ S. Campana, ${ }^{35}$ M. Campanelli, ${ }^{92}$ A. Camplani, ${ }^{39}$ A. Campoverde, ${ }^{148}$ V. Canale, ${ }^{67 \mathrm{a}, 67 \mathrm{~b}}$ M. Cano Bret, ${ }^{58 \mathrm{c}}$ J. Cantero, ${ }^{126}$ T. Cao, ${ }^{158}$ Y. Cao, ${ }^{170}$ M. D. M. Capeans Garrido, ${ }^{35}$ I. Caprini, ${ }^{27 \mathrm{~b}}$ 
M. Caprini, ${ }^{27 b}$ M. Capua, ${ }^{40 b, 40 a}$ R. M. Carbone, ${ }^{38}$ R. Cardarelli, ${ }^{71 a}$ F. C. Cardillo, ${ }^{50}$ I. Carli, ${ }^{140}$ T. Carli, ${ }^{35}$ G. Carlino, ${ }^{67 a}$ B. T. Carlson, ${ }^{136}$ L. Carminati, ${ }^{66 a, 66 b}$ R. M. D. Carney, ${ }^{43 a, 43 b}$ S. Caron, ${ }^{117}$ E. Carquin, ${ }^{144 b}$ S. Carrá, ${ }^{66 a, 66 b}$

G. D. Carrillo-Montoya, ${ }^{35}$ D. Casadei ${ }^{32 b}$ M. P. Casado, ${ }^{14, i}$ A. F. Casha, ${ }^{164}$ M. Casolino, ${ }^{14}$ D. W. Casper, ${ }^{168}$ R. Castelijn, ${ }^{118}$ F. L. Castillo, ${ }^{171}$ V. Castillo Gimenez, ${ }^{171}$ N. F. Castro, ${ }^{137 a, 137 e}$ A. Catinaccio, ${ }^{35}$ J. R. Catmore, ${ }^{131}$ A. Cattai, ${ }^{35}$ J. Caudron, ${ }^{24}$ V. Cavaliere, ${ }^{29}$ E. Cavallaro, ${ }^{14}$ D. Cavalli, ${ }^{66 a}$ M. Cavalli-Sforza, ${ }^{14}$ V. Cavasinni, ${ }^{69 a, 69 b}$ E. Celebi, ${ }^{12 b}$ F. Ceradini, ${ }^{72 a, 72 b}$ L. Cerda Alberich, ${ }^{171}$ A. S. Cerqueira, ${ }^{78 a}$ A. Cerri, ${ }^{153}$ L. Cerrito, ${ }^{71 a, 71 b}$ F. Cerutti, ${ }^{18}$ A. Cervelli, ${ }^{23 b, 23 a}$ S. A. Cetin, ${ }^{12 b}$ A. Chafaq, ${ }^{34 a}$ D. Chakraborty, ${ }^{119}$ S. K. Chan, ${ }^{57}$ W. S. Chan, ${ }^{118}$ Y. L. Chan, ${ }^{61 a}$ P. Chang, ${ }^{170}$ J. D. Chapman, ${ }^{31}$ D. G. Charlton, ${ }^{21}$ C. C. Chau, ${ }^{33}$ C. A. Chavez Barajas, ${ }^{153}$ S. Che, ${ }^{123}$ A. Chegwidden, ${ }^{104}$ S. Chekanov, ${ }^{6}$ S. V. Chekulaev, ${ }^{165 a}$ G. A. Chelkov, ${ }^{77, j}$ M. A. Chelstowska, ${ }^{35}$ C. Chen, ${ }^{58 a}$ C. H. Chen, ${ }^{76}$ H. Chen, ${ }^{29}$ J. Chen, ${ }^{58 a}$ J. Chen, ${ }^{38}$ S. Chen, ${ }^{134}$ S. J. Chen, ${ }^{15 \mathrm{c}}$ X. Chen, ${ }^{15 \mathrm{~b}, \mathrm{k}}$ Y. Chen, ${ }^{80}$ Y-H. Chen, ${ }^{44}$ H. C. Cheng, ${ }^{103}$ H. J. Cheng, ${ }^{15 \mathrm{~d}}$ A. Cheplakov, ${ }^{77}$ E. Cheremushkina, ${ }^{121}$ R. Cherkaoui El Moursli, ${ }^{34 \mathrm{e}}$ E. Cheu, ${ }^{7}$ K. Cheung, ${ }^{62}$ L. Chevalier, ${ }^{142}$ V. Chiarella, ${ }^{49}$ G. Chiarelli, ${ }^{69 a}$ G. Chiodini, ${ }^{65 a}$ A. S. Chisholm, ${ }^{35}$ A. Chitan, ${ }^{27 b}$ I. Chiu, ${ }^{160}$ Y. H. Chiu, ${ }^{173}$ M. V. Chizhov, ${ }^{77}$ K. Choi, ${ }^{63}$ A. R. Chomont, ${ }^{129}$ S. Chouridou, ${ }^{159}$ Y. S. Chow, ${ }^{118}$ V. Christodoulou, ${ }^{92}$ M. C. Chu, ${ }^{61 a}$ J. Chudoba, ${ }^{138}$ A. J. Chuinard, ${ }^{101}$ J. J. Chwastowski, $^{82}$ L. Chytka, $^{127}$ D. Cinca, ${ }^{45}$ V. Cindro, ${ }^{89}$ I. A. Cioară, ${ }^{24}$ A. Ciocio, ${ }^{18}$ F. Cirotto, ${ }^{67 a, 67 b}$ Z. H. Citron, ${ }^{177}$ M. Citterio, ${ }^{66 a}$ A. Clark, ${ }^{52}$ M. R. Clark, ${ }^{38}$ P. J. Clark, ${ }^{48}$ C. Clement, ${ }^{43 a, 43 b}$ Y. Coadou, ${ }^{99}$ M. Cobal, ${ }^{64 a, 64 c}$ A. Coccaro, ${ }^{53 b, 53 a}{ }^{\text {J. Cochran, }}{ }^{76}$ A. E. C. Coimbra, ${ }^{177}$ L. Colasurdo, ${ }^{117}$ B. Cole, ${ }^{38}$ A. P. Colijn, ${ }^{118}$ J. Collot, ${ }^{56}$ P. Conde Muiño, ${ }^{137 a, 137 b}$ E. Coniavitis, ${ }^{50}$ S. H. Connell, ${ }^{32 b}$ I. A. Connelly, ${ }^{98}$ S. Constantinescu, ${ }^{27 b}$ F. Conventi, ${ }^{67 a, 1}$ A. M. Cooper-Sarkar, ${ }^{132}$ F. Cormier, ${ }^{172}$ K. J. R. Cormier, ${ }^{164}$ M. Corradi, ${ }^{70 a, 70 b}$ E. E. Corrigan, ${ }^{94}$ F. Corriveau, ${ }^{101, m}$ A. Cortes-Gonzalez, ${ }^{35}$ M. J. Costa, ${ }^{171}$ D. Costanzo, ${ }^{146}$ G. Cottin, ${ }^{31}$ G. Cowan, ${ }^{91}$ B. E. Cox, ${ }^{98}$ J. Crane, ${ }^{98}$ K. Cranmer, ${ }^{122}$ S. J. Crawley, ${ }^{55}$ R. A. Creager, ${ }^{134}$ G. Cree, ${ }^{33}$ S. Crépé-Renaudin, ${ }^{56}$ F. Crescioli, ${ }^{133}$ M. Cristinziani, ${ }^{24}$ V. Croft, ${ }^{122}$ G. Crosetti, ${ }^{40 b, 40 a}$ A. Cueto, ${ }^{96}$

T. Cuhadar Donszelmann, ${ }^{146}$ A. R. Cukierman, ${ }^{150}$ M. Curatolo, ${ }^{49}$ J. Cúth, ${ }^{97}$ S. Czekierda, ${ }^{82}$ P. Czodrowski, ${ }^{35}$ M. J. Da Cunha Sargedas De Sousa, ${ }^{58 b}$ C. Da Via, ${ }^{98}$ W. Dabrowski, ${ }^{81 a}$ T. Dado, ${ }^{28 a, n}$ S. Dahbi, ${ }^{34 e}$ T. Dai, ${ }^{103}$ F. Dallaire, ${ }^{107}$ C. Dallapiccola, ${ }^{100}$ M. Dam, ${ }^{39}$ G. D'amen, ${ }^{23 b, 23 a}$ J. Damp, ${ }^{97}$ J. R. Dandoy, ${ }^{134}$ M. F. Daneri, ${ }^{30}$ N. P. Dang, ${ }^{178, f}$ N. D. Dann, ${ }^{98}$ M. Danninger, ${ }^{172}$ V. Dao, ${ }^{35}$ G. Darbo, ${ }^{53 b}$ S. Darmora, ${ }^{8}$ O. Dartsi, ${ }^{5}$ A. Dattagupta, ${ }^{128}$ T. Daubney, ${ }^{44}$ S. D' Auria, ${ }^{55}$ W. Davey, ${ }^{24}$ C. David, ${ }^{44}$ T. Davidek, ${ }^{140}$ D. R. Davis, ${ }^{47}$ E. Dawe, ${ }^{102}$ I. Dawson, ${ }^{146}$ K. De, ${ }^{8}$ R. De Asmundis, ${ }^{67 a}$ A. De Benedetti, ${ }^{125}$ S. De Castro, ${ }^{23 b, 23 a}$ S. De Cecco, ${ }^{70 a, 70 b}$ N. De Groot, ${ }^{117}$ P. de Jong, ${ }^{118}$ H. De la Torre, ${ }^{104}$ F. De Lorenzi, ${ }^{76}$ A. De Maria, ${ }^{51, o}$ D. De Pedis, ${ }^{70 a}$ A. De Salvo, ${ }^{70 a}$ U. De Sanctis, ${ }^{71 a, 71 b}$ A. De Santo, ${ }^{153}$ K. De Vasconcelos Corga, ${ }^{99}$ J. B. De Vivie De Regie, ${ }^{129}$ C. Debenedetti, ${ }^{143}$ D. V. Dedovich, ${ }^{77}$ N. Dehghanian, ${ }^{3}$ M. Del Gaudio, ${ }^{40 b, 40 a}$ J. Del Peso, ${ }^{96}$ D. Delgove, ${ }^{129}$ F. Deliot, ${ }^{142}$ C. M. Delitzsch, ${ }^{7}$ M. Della Pietra, ${ }^{67 a, 67 b}$ D. Della Volpe, ${ }^{52}$ A. Dell'Acqua, ${ }^{35}$ L. Dell' Asta, ${ }^{25}$ M. Delmastro, ${ }^{5}$ C. Delporte, ${ }^{129}$ P. A. Delsart, ${ }^{56}$ D. A. DeMarco, ${ }^{164}$ S. Demers, ${ }^{180}$ M. Demichev, ${ }^{77}$ S. P. Denisov, ${ }^{121}$ D. Denysiuk, ${ }^{118}$ L. D’Eramo, ${ }^{133}$ D. Derendarz, ${ }^{82}$ J. E. Derkaoui, ${ }^{34 d}$ F. Derue, ${ }^{133}$ P. Dervan, ${ }^{88}$ K. Desch, ${ }^{24}$ C. Deterre, ${ }^{44}$ K. Dette, ${ }^{164}$ M. R. Devesa, ${ }^{30}$ P. O. Deviveiros, ${ }^{35}$ A. Dewhurst, ${ }^{141}$ S. Dhaliwal, ${ }^{26}$ F. A. Di Bello, ${ }^{52}$ A. Di Ciaccio, ${ }^{71 a, 71 b}$ L. Di Ciaccio, ${ }^{5}$

W. K. Di Clemente, ${ }^{134}$ C. Di Donato, ${ }^{67 a, 67 b}$ A. Di Girolamo, ${ }^{35}$ B. Di Micco, ${ }^{72 a, 72 b}$ R. Di Nardo, ${ }^{35}$ K. F. Di Petrillo, ${ }^{57}$ A. Di Simone, ${ }^{50}$ R. Di Sipio, ${ }^{164}$ D. Di Valentino, ${ }^{33}$ C. Diaconu, ${ }^{99}$ M. Diamond, ${ }^{164}$ F. A. Dias, ${ }^{39}$ T. Dias Do Vale, ${ }^{137 a}$ M. A. Diaz, ${ }^{144 a}$ J. Dickinson, ${ }^{18}$ E. B. Diehl, ${ }^{103}$ J. Dietrich, ${ }^{19}$ S. Díez Cornell, ${ }^{44}$ A. Dimitrievska, ${ }^{18}$ J. Dingfelder, ${ }^{24}$ F. Dittus, ${ }^{35}$ F. Djama, ${ }^{99}$ T. Djobava, ${ }^{156 b}$ J. I. Djuvsland, ${ }^{59}$ M. A. B. Do Vale, ${ }^{78 c}$ M. Dobre, ${ }^{27 b}$ D. Dodsworth, ${ }^{26}$ C. Doglioni, ${ }^{94}$ J. Dolejsi, ${ }^{140}$ Z. Dolezal, ${ }^{140}$ M. Donadelli, ${ }^{78 d}$ J. Donini, ${ }^{37}$ A. D'onofrio, ${ }^{90}$ M. D'Onofrio, ${ }^{88}$ J. Dopke, ${ }^{141}$ A. Doria, ${ }^{67 a}$ M. T. Dova, ${ }^{86}$ A. T. Doyle, ${ }^{55}$ E. Drechsler, ${ }^{51}$ E. Dreyer, ${ }^{149}$ T. Dreyer, ${ }^{51}$ M. Dris, ${ }^{10}$ Y. Du, ${ }^{58 b}$ J. Duarte-Campderros, ${ }^{158}$ F. Dubinin, ${ }^{108}$ A. Dubreuil, ${ }^{52}$ E. Duchovni, ${ }^{177}$ G. Duckeck, ${ }^{112}$ A. Ducourthial, ${ }^{133}$ O. A. Ducu, ${ }^{107, p}$ D. Duda, ${ }^{113}$ A. Dudarev, ${ }^{35}$ A. C. Dudder, ${ }^{97}$ E. M. Duffield, ${ }^{18}$ L. Duflot, ${ }^{129}$ M. Dührssen, ${ }^{35}$ C. Dülsen, ${ }^{179}$ M. Dumancic, ${ }^{177}$ A. E. Dumitriu, ${ }^{27 b, q}$ A. K. Duncan, ${ }^{55}$ M. Dunford, ${ }^{59}$ A. Duperrin, ${ }^{99}$ H. Duran Yildiz, ${ }^{4 a}$ M. Düren, ${ }^{54}$ A. Durglishvili, ${ }^{156 b}$ D. Duschinger, ${ }^{46}$ B. Dutta, ${ }^{44}$ D. Duvnjak, ${ }^{1}$ M. Dyndal, ${ }^{44}$ S. Dysch, ${ }^{98}$ B. S. Dziedzic, ${ }^{82}$ C. Eckardt,${ }^{44}$ K. M. Ecker, ${ }^{113}$ R. C. Edgar, ${ }^{103}$ T. Eifert, ${ }^{35}$ G. Eigen, ${ }^{17}$ K. Einsweiler, ${ }^{18}$ T. Ekelof, ${ }^{169}$ M. El Kacimi, ${ }^{34 c}$ R. El Kosseifi, ${ }^{99}$ V. Ellajosyula, ${ }^{99}$ M. Ellert, ${ }^{169}$ F. Ellinghaus, ${ }^{179}$ A. A. Elliot, ${ }^{90}$ N. Ellis, ${ }^{35}$ J. Elmsheuser, ${ }^{29}$ M. Elsing, ${ }^{35}$ D. Emeliyanov, ${ }^{141}$ Y. Enari, ${ }^{160}$ J. S. Ennis, ${ }^{175}$ M. B. Epland, ${ }^{47}$ J. Erdmann, ${ }^{45}$ A. Ereditato, ${ }^{20}$ S. Errede, ${ }^{170}$ M. Escalier, ${ }^{129}$ C. Escobar, ${ }^{171}$ B. Esposito, ${ }^{49}$ O. Estrada Pastor, ${ }^{171}$ A. I. Etienvre, ${ }^{142}$ E. Etzion, ${ }^{158}$ H. Evans, ${ }^{63}$ A. Ezhilov, ${ }^{135}$ M. Ezzi, ${ }^{34 \mathrm{e}}$ F. Fabbri, ${ }^{55}$ L. Fabbri, ${ }^{23 b, 23 a}$ V. Fabiani, ${ }^{117}$ G. Facini, ${ }^{92}$ R. M. Faisca Rodrigues Pereira, ${ }^{137 a}$ R. M. Fakhrutdinov, ${ }^{121}$ S. Falciano, ${ }^{70 a}$ P. J. Falke, ${ }^{5}$ S. Falke, ${ }^{5}$ J. Faltova, ${ }^{140}$ Y. Fang, ${ }^{15}$ M. Fanti, ${ }^{66 a, 66 b}$ A. Farbin, ${ }^{8}$ A. Farilla, ${ }^{72 a}$ E. M. Farina, ${ }^{68 a, 68 b}$ T. Farooque, ${ }^{104}$ S. Farrell, ${ }^{18}$ S. M. Farrington, ${ }^{175}$ P. Farthouat, ${ }^{35}$

F. Fassi, ${ }^{34 \mathrm{e}}$ P. Fassnacht, ${ }^{35}$ D. Fassouliotis, ${ }^{9}$ M. Faucci Giannelli, ${ }^{48}$ A. Favareto, ${ }^{53 b, 53 a}$ W. J. Fawcett, ${ }^{52}$ L. Fayard, ${ }^{129}$ 
O. L. Fedin, ${ }^{135, \mathrm{r}}$ W. Fedorko, ${ }^{172}$ M. Feickert, ${ }^{41}$ S. Feigl, ${ }^{131}$ L. Feligioni, ${ }^{99}$ C. Feng, ${ }^{58 b}$ E. J. Feng,${ }^{35}$ M. Feng, ${ }^{47}$ M. J. Fenton, ${ }^{55}$ A. B. Fenyuk, ${ }^{121}$ L. Feremenga, ${ }^{8}$ J. Ferrando, ${ }^{44}$ A. Ferrari, ${ }^{169}$ P. Ferrari, ${ }^{118}$ R. Ferrari, ${ }^{68 \mathrm{a}}$ D. E. Ferreira de Lima, ${ }^{59 \mathrm{~b}}$ A. Ferrer, ${ }^{171}$ D. Ferrere, ${ }^{52}$ C. Ferretti, ${ }^{103}$ F. Fiedler, ${ }^{97}$ A. Filipčičc, ${ }^{89}$ F. Filthaut, ${ }^{117}$ K. D. Finelli, ${ }^{25}$ M. C. N. Fiolhais, ${ }^{137 a, 137 c, s}$ L. Fiorini, ${ }^{171}$ C. Fischer, ${ }^{14}$ W. C. Fisher, ${ }^{104}$ N. Flaschel, ${ }^{44}$ I. Fleck, ${ }^{148}$ P. Fleischmann, ${ }^{103}$ R. R. M. Fletcher, ${ }^{134}$ T. Flick, ${ }^{179}$ B. M. Flierl, ${ }^{112}$ L. M. Flores, ${ }^{134}$ L. R. Flores Castillo, ${ }^{61 \mathrm{a}}$ N. Fomin, ${ }^{17}$ G. T. Forcolin, ${ }^{98}$ A. Formica, ${ }^{142}$ F. A. Förster, ${ }^{14}$ A. C. Forti, ${ }^{98}$ A. G. Foster, ${ }^{21}$ D. Fournier, ${ }^{129}$ H. Fox, ${ }^{87}$ S. Fracchia, ${ }^{146}$ P. Francavilla, ${ }^{69 a, 69 b}$ M. Franchini, ${ }^{23 b, 23 a}$ S. Franchino, ${ }^{59 \mathrm{a}}$ D. Francis, ${ }^{35}$ L. Franconi, ${ }^{131}$ M. Franklin, ${ }^{57}$ M. Frate, ${ }^{168}$ M. Fraternali, ${ }^{68 a, 68 b}$ D. Freeborn, ${ }^{92}$ S. M. Fressard-Batraneanu, ${ }^{35}$ B. Freund, ${ }^{107}$ W. S. Freund, ${ }^{78 b}$ D. Froidevaux,${ }^{35}$ J. A. Frost,${ }^{132}$ C. Fukunaga, ${ }^{161}$ T. Fusayasu, ${ }^{114}$ J. Fuster, ${ }^{171}$ O. Gabizon, ${ }^{157}$ A. Gabrielli, ${ }^{23 b, 23 a}$ A. Gabrielli, ${ }^{18}$ G. P. Gach ${ }^{81 a}$ S. Gadatsch,${ }^{52}$ P. Gadow, ${ }^{113}$ G. Gagliardi, ${ }^{53 b, 53 a}$ L. G. Gagnon, ${ }^{107}$ C. Galea, ${ }^{27 b}$ B. Galhardo, ${ }^{137 a, 137 \mathrm{c}}$ E. J. Gallas, ${ }^{132}$ B. J. Gallop, ${ }^{141}$ P. Gallus, ${ }^{139}$ G. Galster, ${ }^{39}$ R. Gamboa Goni, ${ }^{90}$ K. K. Gan, ${ }^{123}$ S. Ganguly, ${ }^{177}$ Y. Gao, ${ }^{88}$ Y. S. Gao, ${ }^{150, g}$ C. García, ${ }^{171}$ J. E. García Navarro, ${ }^{171}$ J. A. García Pascual, ${ }^{15 a}$ M. Garcia-Sciveres, ${ }^{18}$ R. W. Gardner, ${ }^{36}$ N. Garelli, ${ }^{150}$ V. Garonne, ${ }^{131}$ K. Gasnikova, ${ }^{44}$ A. Gaudiello, ${ }^{53 b, 53 a}$ G. Gaudio, ${ }^{68 a}$ I. L. Gavrilenko, ${ }^{108}$ A. Gavrilyuk, ${ }^{109}$ C. Gay, ${ }^{172}$ G. Gaycken, ${ }^{24}$ E. N. Gazis,${ }^{10}$ C. N. P. Gee,${ }^{141}$ J. Geisen, ${ }^{51}$ M. Geisen, ${ }^{97}$ M. P. Geisler, ${ }^{59 a}$ K. Gellerstedt, ${ }^{43 a, 43 b}$ C. Gemme, ${ }^{53 b}$ M. H. Genest, ${ }^{56}$ C. Geng, ${ }^{103}$ S. Gentile, ${ }^{70 a, 70 b}$ C. Gentsos, ${ }^{159}$ S. George,${ }^{91}$ D. Gerbaudo, ${ }^{14}$ G. Gessner, ${ }^{45}$ S. Ghasemi, ${ }^{148}$ M. Ghasemi Bostanabad, ${ }^{173}$ M. Ghneimat, ${ }^{24}$ B. Giacobbe, ${ }^{23 b}$ S. Giagu, ${ }^{70 a, 70 b}$ N. Giangiacomi, ${ }^{23 b, 23 a}$ P. Giannetti, ${ }^{69}$ S. M. Gibson, ${ }^{91}$ M. Gignac, ${ }^{143}$ D. Gillberg, ${ }^{33}$ G. Gilles, ${ }^{179}$ D. M. Gingrich, ${ }^{3, e}$ M. P. Giordani, ${ }^{64 a, 64 c}$ F. M. Giorgi, ${ }^{23 b}$ P. F. Giraud, ${ }^{142}$ P. Giromini, ${ }^{57}$ G. Giugliarelli, ${ }^{64,64 c}$ D. Giugni, ${ }^{66 a}$ F. Giuli, ${ }^{132}$ M. Giulini, ${ }^{59 b}$ S. Gkaitatzis, ${ }^{159}$ I. Gkialas, ${ }^{9, \mathrm{t}}$ E. L. Gkougkousis, ${ }^{14}$ P. Gkountoumis, ${ }^{10}$ L. K. Gladilin, ${ }^{111}$ C. Glasman, ${ }^{96}$ J. Glatzer, ${ }^{14}$ P. C. F. Glaysher, ${ }^{44}$ A. Glazov, ${ }^{44}$ M. Goblirsch-Kolb, ${ }^{26}$ J. Godlewski, ${ }^{82}$ S. Goldfarb, ${ }^{102}$ T. Golling, ${ }^{52}$ D. Golubkov, ${ }^{121}$ A. Gomes, ${ }^{177 a, 137 b, 137 \mathrm{~d}}$ R. Goncalves Gama, ${ }^{78 a}$ R. Gonçalo, ${ }^{137 a}$ G. Gonella, ${ }^{50}$ L. Gonella, ${ }^{21}$ A. Gongadze, ${ }^{77}$ F. Gonnella, ${ }^{21}$ J. L. Gonski, ${ }^{57}$ S. González de la Hoz, ${ }^{171}$ S. Gonzalez-Sevilla, ${ }^{52}$ L. Goossens, ${ }^{35}$ P. A. Gorbounov, ${ }^{109}$ H. A. Gordon, ${ }^{29}$ B. Gorini, ${ }^{35}$ E. Gorini, ${ }^{65 a, 65 b}$ A. Gorišek,${ }^{89}$ A. T. Goshaw, ${ }^{47}$ C. Gössling, ${ }^{45}$ M. I. Gostkin, ${ }^{77}$ C. A. Gottardo,${ }^{24}$ C. R. Goudet, ${ }^{129}$ D. Goujdami, ${ }^{34 c}$ A. G. Goussiou, ${ }^{145}$ N. Govender, ${ }^{32 b, u}$ C. Goy, ${ }^{5}$ E. Gozani, ${ }^{157}$ I. Grabowska-Bold, ${ }^{81 a}$ P. O. J. Gradin, ${ }^{169}$ E. C. Graham, ${ }^{88}$ J. Gramling, ${ }^{168}$ E. Gramstad, ${ }^{131}$ S. Grancagnolo, ${ }^{19}$ V. Gratchev, ${ }^{135}$ P. M. Gravila, ${ }^{27 f}$ C. Gray,${ }^{55}$ H. M. Gray, ${ }^{18}$ Z. D. Greenwood, ${ }^{93, v}$ C. Grefe, ${ }^{24}$ K. Gregersen, ${ }^{92}$ I. M. Gregor, ${ }^{44}$ P. Grenier, ${ }^{150}$ K. Grevtsov, ${ }^{44}$ J. Griffiths, ${ }^{8}$ A. A. Grillo, ${ }^{143}$ K. Grimm, ${ }^{150, w}$ S. Grinstein, ${ }^{14, x}$ Ph. Gris ${ }^{37}$ J.-F. Grivaz, ${ }^{129}$ S. Groh, ${ }^{97}$ E. Gross,${ }^{177}$ J. Grosse-Knetter, ${ }^{51}$ G. C. Grossi, ${ }^{93}$ Z. J. Grout, ${ }^{92}$ C. Grud, ${ }^{103}$ A. Grummer, ${ }^{116}$ L. Guan, ${ }^{103}$ W. Guan, ${ }^{178}$ J. Guenther, ${ }^{35}$ A. Guerguichon, ${ }^{129}$ F. Guescini, ${ }^{165 a}$ D. Guest, ${ }^{168}$ R. Gugel, ${ }^{50}$ B. Gui, ${ }^{123}$ T. Guillemin, ${ }^{5}$ S. Guindon, ${ }^{35}$ U. Gul, ${ }^{55}$ C. Gumpert, ${ }^{35}$ J. Guo, ${ }^{58 c}$ W. Guo, ${ }^{103}$ Y. Guo, ${ }^{58, y}$ Z. Guo, ${ }^{99}$ R. Gupta, ${ }^{41}$ S. Gurbuz, ${ }^{12 \mathrm{c}}$ G. Gustavino, ${ }^{125}$ B. J. Gutelman, ${ }^{157}$ P. Gutierrez, ${ }^{125}$ C. Gutschow, ${ }^{92}$ C. Guyot, ${ }^{142}$ M. P. Guzik, ${ }^{81 a}$ C. Gwenlan, ${ }^{132}$ C. B. Gwilliam, ${ }^{88}$ A. Haas, ${ }^{122}$ C. Haber, ${ }^{18}$ H. K. Hadavand, ${ }^{8}$ N. Haddad, ${ }^{34 \mathrm{e}}$ A. Hadef, ${ }^{58 a}$ S. Hageböck, ${ }^{24}$ M. Hagihara, ${ }^{166}$ H. Hakobyan, ${ }^{181, a}$ M. Haleem, ${ }^{174}$ J. Haley, ${ }^{126}$ G. Halladjian, ${ }^{104}$ G. D. Hallewell, ${ }^{99}$ K. Hamacher, ${ }^{179}$ P. Hamal, ${ }^{127}$ K. Hamano, ${ }^{173}$ A. Hamilton, ${ }^{32 \mathrm{a}}$ G. N. Hamity, ${ }^{146}$ K. Han ${ }^{58 \mathrm{a}, \mathrm{z}}$ L. Han, ${ }^{58 \mathrm{a}}$ S. Han, ${ }^{15 \mathrm{~d}}$ K. Hanagaki, ${ }^{79, \text { aa }}$ M. Hance, ${ }^{143}$ D. M. Handl, ${ }^{112}$ B. Haney, ${ }^{134}$ R. Hankache, ${ }^{133}$ P. Hanke, ${ }^{59 a}$ E. Hansen, ${ }^{94}$ J. B. Hansen, ${ }^{39}$ J. D. Hansen, ${ }^{39}$ M. C. Hansen, ${ }^{24}$ P. H. Hansen, ${ }^{39}$ K. Hara, ${ }^{166}$ A. S. Hard ${ }^{178}$ T. Harenberg, ${ }^{179}$ S. Harkusha, ${ }^{105}$ P. F. Harrison, ${ }^{175}$ N. M. Hartmann, ${ }^{112}$ Y. Hasegawa, ${ }^{147}$ A. Hasib, ${ }^{48}$ S. Hassani, ${ }^{142}$ S. Haug, ${ }^{20}$ R. Hauser, ${ }^{104}$ L. Hauswald, ${ }^{46}$ L. B. Havener, ${ }^{38}$ M. Havranek, ${ }^{139}$ C. M. Hawkes, ${ }^{21}$ R. J. Hawkings,${ }^{35}$ D. Hayden, ${ }^{104}$ C. Hayes, ${ }^{152}$ C. P. Hays,${ }^{132}$ J. M. Hays, ${ }^{90}$ H. S. Hayward, ${ }^{88}$ S. J. Haywood, ${ }^{141}$ M. P. Heath, ${ }^{48}$ V. Hedberg, ${ }^{94}$ L. Heelan, ${ }^{8}$ S. Heer, ${ }^{24}$ K. K. Heidegger, ${ }^{50}$ J. Heilman, ${ }^{33}$ S. Heim, ${ }^{44}$ T. Heim, ${ }^{18}$ B. Heinemann, ${ }^{44, b b}$ J. J. Heinrich, ${ }^{112}$ L. Heinrich, ${ }^{122}$ C. Heinz,${ }^{54}$ J. Hejbal, ${ }^{138}$ L. Helary, ${ }^{35}$ A. Held, ${ }^{172}$ S. Hellesund, ${ }^{131}$ S. Hellman, ${ }^{43 a, 43 b}$ C. Helsens,${ }^{35}$ R. C. W. Henderson, ${ }^{87}$ Y. Heng, ${ }^{178}$ S. Henkelmann, ${ }^{172}$ A. M. Henriques Correia, ${ }^{35}$ G. H. Herbert, ${ }^{19}$ H. Herde, ${ }^{26}$ V. Herget, ${ }^{174}$ Y. Hernández Jiménez, ${ }^{32 c}$ H. Herr, ${ }^{97}$ G. Herten, ${ }^{50}$ R. Hertenberger, ${ }^{112}$ L. Hervas, ${ }^{35}$ T. C. Herwig, ${ }^{134}$ G. G. Hesketh ${ }^{92}$ N. P. Hessey, ${ }^{165 a}$ J. W. Hetherly, ${ }^{41}$ S. Higashino, ${ }^{79}$ E. Higón-Rodriguez,${ }^{171}$ K. Hildebrand,${ }^{36}$ E. Hill, ${ }^{173}$ J. C. Hill, ${ }^{31}$ K. K. Hill, ${ }^{29}$ K. H. Hiller ${ }^{44}$ S. J. Hillier,${ }^{21}$ M. Hils, ${ }^{46}$ I. Hinchliffe, ${ }^{18}$ M. Hirose, ${ }^{130}$ D. Hirschbuehl, ${ }^{179}$ B. Hiti, ${ }^{89}$ O. Hladik, ${ }^{138}$ D. R. Hlaluku, ${ }^{32 c}$ X. Hoad ${ }^{48}$ J. Hobbs, ${ }^{152}$ N. Hod, ${ }^{165 a}$ M. C. Hodgkinson, ${ }^{146}$ A. Hoecker, ${ }^{35}$ M. R. Hoeferkamp, ${ }^{116}$ F. Hoenig, ${ }^{112}$ D. Hohn,${ }^{24}$ D. Hohov, ${ }^{129}$ T. R. Holmes, ${ }^{36}$ M. Holzbock, ${ }^{112}$ M. Homann, ${ }^{45}$ S. Honda, ${ }^{166}$ T. Honda, ${ }^{79}$ T. M. Hong, ${ }^{136}$ A. Hönle, ${ }^{113}$ B. H. Hooberman, ${ }^{170}$ W. H. Hopkins ${ }^{128}$ Y. Horii, ${ }^{15}$ P. Horn ${ }^{46}$ A. J. Horton, ${ }^{149}$ L. A. Horyn, ${ }^{36}$ J-Y. Hostachy, ${ }^{56}$ A. Hostiuc, ${ }^{145}$ S. Hou, ${ }^{155}$ A. Hoummada, ${ }^{34 \mathrm{a}}$ J. Howarth, ${ }^{98}$ J. Hoya, ${ }^{86}$ M. Hrabovsky, ${ }^{127}$ J. Hrdinka, ${ }^{35}$ I. Hristova, ${ }^{19}$ J. Hrivnac, ${ }^{129}$ A. Hrynevich, ${ }^{106}$ T. Hryn'ova, ${ }^{5}$ P. J. Hsu, ${ }^{62}$ S.-C. Hsu, ${ }^{145}$ Q. Hu, ${ }^{29}$ S. Hu, ${ }^{58 \mathrm{c}}$ Y. Huang, ${ }^{15 \mathrm{a}}$ Z. Hubacek, ${ }^{139}$ F. Hubaut, ${ }^{99}$ M. Huebner, ${ }^{24}$ 
F. Huegging, ${ }^{24}$ T. B. Huffman, ${ }^{132}$ E. W. Hughes, ${ }^{38}$ M. Huhtinen, ${ }^{35}$ R. F. H. Hunter, ${ }^{33}$ P. Huo, ${ }^{152}$ A. M. Hupe, ${ }^{33}$ N. Huseynov, ${ }^{77, \mathrm{~d}}$ J. Huston, ${ }^{104} \mathrm{~J}$. Huth, ${ }^{57}$ R. Hyneman, ${ }^{103}$ G. Iacobucci, ${ }^{52}$ G. Iakovidis, ${ }^{29}$ I. Ibragimov, ${ }^{148}$ L. Iconomidou-Fayard, ${ }^{129}$ Z. Idrissi, ${ }^{34 \mathrm{e}}$ P. Iengo, ${ }^{35}$ R. Ignazzi, ${ }^{39}$ O. Igonkina, ${ }^{18, c c}$ R. Iguchi, ${ }^{160}$ T. Iizawa, ${ }^{52}$ Y. Ikegami, ${ }^{79}$ M. Ikeno, ${ }^{79}$ D. Iliadis, ${ }^{159}$ N. Ilic, ${ }^{150}$ F. Iltzsche, ${ }^{46}$ G. Introzzi, ${ }^{68 a, 68 b}$ M. Iodice, ${ }^{72 a}$ K. Iordanidou, ${ }^{38}$ V. Ippolito, ${ }^{70 a, 70 b}$ M. F. Isacson, ${ }^{169} \mathrm{~N}$. Ishijima, ${ }^{130} \mathrm{M}$. Ishino, ${ }^{160} \mathrm{M}$. Ishitsuka, ${ }^{162} \mathrm{C}$. Issever, ${ }^{132} \mathrm{~S}$. Istin, ${ }^{12 \mathrm{c}, \mathrm{dd}} \mathrm{F}$. Ito, ${ }^{166} \mathrm{~J}$. M. Iturbe Ponce, ${ }^{61 \mathrm{a}}$ R. Iuppa ${ }^{73 a, 73 b}$ A. Ivina, ${ }^{177}$ H. Iwasaki, ${ }^{79}$ J. M. Izen, ${ }^{42}$ V. Izzo, ${ }^{67 a}$ S. Jabbar, ${ }^{3}$ P. Jacka, ${ }^{138}$ P. Jackson, ${ }^{1}$ R. M. Jacobs, ${ }^{24}$ V. Jain, ${ }^{2}$ G. Jäkel, ${ }^{179}$ K. B. Jakobi,${ }^{97}$ K. Jakobs,${ }^{50}$ S. Jakobsen, ${ }^{74}$ T. Jakoubek, ${ }^{138}$ D. O. Jamin, ${ }^{126}$ D. K. Jana, ${ }^{93}$ R. Jansky, ${ }^{52}$ J. Janssen, ${ }^{24}$ M. Janus, ${ }^{51}$ P. A. Janus, ${ }^{81 a}$ G. Jarlskog, ${ }^{94}$ N. Javadov, ${ }^{77, d}$ T. Javůrek, ${ }^{50}$ M. Javurkova, ${ }^{50}$ F. Jeanneau, ${ }^{142}$ L. Jeanty, ${ }^{18}$ J. Jejelava, ${ }^{156 a, e e}$ A. Jelinskas, ${ }^{175}$ P. Jenni, ${ }^{50, f f}$ J. Jeong, ${ }^{44}$ C. Jeske, ${ }^{175}$ S. Jézéquel, ${ }^{5} \mathrm{H}$. Ji, ${ }^{178}$ J. Jia, ${ }^{152}$ H. Jiang,${ }^{76}$ Y. Jiang, ${ }^{58 \mathrm{a}}$ Z. Jiang, ${ }^{150, \mathrm{gg}} \mathrm{S}$. Jiggins, ${ }^{50}$ F. A. Jimenez Morales, ${ }^{37}$ J. Jimenez Pena, ${ }^{171}$ S. Jin, ${ }^{15 \mathrm{c}}$ A. Jinaru, ${ }^{27 \mathrm{~b}}$ O. Jinnouchi, ${ }^{162}$ H. Jivan, ${ }^{32 \mathrm{c}}$ P. Johansson, ${ }^{146}$ K. A. Johns, ${ }^{7}$ C. A. Johnson, ${ }^{63}$ W. J. Johnson, ${ }^{145}$ K. Jon-And, ${ }^{43 a, 43 b}$ R. W. L. Jones, ${ }^{87}$ S. D. Jones, ${ }^{153}$ S. Jones, ${ }^{7}$ T. J. Jones ${ }^{88}$ J. Jongmanns, ${ }^{59 a}$ P. M. Jorge, ${ }^{137 a, 137 b}$ J. Jovicevic, ${ }^{165 a}$ X. Ju, ${ }^{178}$ J. J. Junggeburth, ${ }^{113}$ A. Juste Rozas, ${ }^{14, x}$ A. Kaczmarska, ${ }^{82}$ M. Kado, ${ }^{129}$ H. Kagan, ${ }^{123}$ M. Kagan, ${ }^{150}$ T. Kaji, ${ }^{176}$ E. Kajomovitz, ${ }^{157}$ C. W. Kalderon, ${ }^{94}$ A. Kaluza, ${ }^{97}$ S. Kama, ${ }^{41}$ A. Kamenshchikov, ${ }^{121}$ L. Kanjir, ${ }^{89}$ Y. Kano, ${ }^{160}$ V. A. Kantserov, ${ }^{110}$ J. Kanzaki, ${ }^{79}$

B. Kaplan, ${ }^{122}$ L. S. Kaplan, ${ }^{178}$ D. Kar, ${ }^{32 \mathrm{c}}$ M. J. Kareem, ${ }^{165 \mathrm{~b}}$ E. Karentzos, ${ }^{10}$ S. N. Karpov, ${ }^{77}$ Z. M. Karpova, ${ }^{77}$

V. Kartvelishvili, ${ }^{87}$ A. N. Karyukhin, ${ }^{121}$ K. Kasahara, ${ }^{166}$ L. Kashif, ${ }^{178}$ R. D. Kass, ${ }^{123}$ A. Kastanas, ${ }^{151}$ Y. Kataoka, ${ }^{160}$ C. Kato, ${ }^{160}$ J. Katzy, ${ }^{44}$ K. Kawade ${ }^{80}$ K. Kawagoe, ${ }^{85}$ T. Kawamoto, ${ }^{160}$ G. Kawamura, ${ }^{51}$ E. F. Kay, ${ }^{88}$ V. F. Kazanin, ${ }^{120 b, 120 a}$ R. Keeler, ${ }^{173}$ R. Kehoe, ${ }^{41}$ J. S. Keller,${ }^{33}$ E. Kellermann, ${ }^{94}$ J. J. Kempster, ${ }^{21}$ J. Kendrick, ${ }^{21}$ O. Kepka, ${ }^{138}$ S. Kersten, ${ }^{179}$

B. P. Kerševan ${ }^{89}$ R. A. Keyes, ${ }^{101}$ M. Khader, ${ }^{170}$ F. Khalil-Zada, ${ }^{13}$ A. Khanov, ${ }^{126}$ A. G. Kharlamov, ${ }^{120 b, 120 a}$ T. Kharlamova, ${ }^{120 b, 120 \mathrm{a}}$ A. Khodinov, ${ }^{163}$ T. J. Khoo, ${ }^{52}$ E. Khramov, ${ }^{77}$ J. Khubua, ${ }^{156 \mathrm{~b}}$ S. Kido, ${ }^{80}$ M. Kiehn, ${ }^{52}$ C. R. Kilby, ${ }^{91}$ S. H. Kim, ${ }^{166}$ Y. K. Kim, ${ }^{36}$ N. Kimura, ${ }^{64,64 c}$ O. M. Kind,${ }^{19}$ B. T. King, ${ }^{88}$ D. Kirchmeier, ${ }^{46}$ J. Kirk, ${ }^{141}$ A. E. Kiryunin, ${ }^{113}$ T. Kishimoto, ${ }^{160}$ D. Kisielewska, ${ }^{81 a}$ V. Kitali, ${ }^{44}$ O. Kivernyk, ${ }^{5}$ E. Kladiva, ${ }^{28 b, a}$ T. Klapdor-Kleingrothaus, ${ }^{50}$ M. H. Klein, ${ }^{103}$ M. Klein, ${ }^{88}$ U. Klein ${ }^{88}$ K. Kleinknecht, ${ }^{97}$ P. Klimek, ${ }^{19}$ A. Klimentov,${ }^{29}$ R. Klingenberg, ${ }^{45, a}$ T. Klingl, ${ }^{24}$

T. Klioutchnikova, ${ }^{35}$ F. F. Klitzner, ${ }^{112}$ P. Kluit, ${ }^{118}$ S. Kluth,${ }^{113}$ E. Kneringer, ${ }^{74}$ E. B. F. G. Knoops, ${ }^{99}$ A. Knue, ${ }^{50}$ A. Kobayashi, ${ }^{160}$ D. Kobayashi, ${ }^{85}$ T. Kobayashi, ${ }^{160}$ M. Kobel, ${ }^{46}$ M. Kocian, ${ }^{150}$ P. Kodys, ${ }^{140}$ T. Koffas, ${ }^{33}$ E. Koffeman, ${ }^{118}$ N. M. Köhler, ${ }^{113}$ T. Koi, ${ }^{150}$ M. Kolb, ${ }^{59 b}$ I. Koletsou, ${ }^{5}$ T. Kondo, ${ }^{79}$ N. Kondrashova, ${ }^{58 c}$ K. Köneke, ${ }^{50}$ A. C. König, ${ }^{117}$ T. Kono, ${ }^{79}$ R. Konoplich, ${ }^{122, \text { hh }}$ V. Konstantinides, ${ }^{92}$ N. Konstantinidis, ${ }^{92}$ B. Konya ${ }^{94}$ R. Kopeliansky,${ }^{63}$ S. Koperny, ${ }^{81 a}$ K. Korcyl,${ }^{82}$ K. Kordas, ${ }^{159}$ A. Korn, ${ }^{92}$ I. Korolkov, ${ }^{14}$ E. V. Korolkova, ${ }^{146}$ O. Kortner, ${ }^{113}$ S. Kortner, ${ }^{113}$ T. Kosek, ${ }^{140}$ V. V. Kostyukhin, ${ }^{24}$ A. Kotwal, ${ }^{47}$ A. Koulouris, ${ }^{10}$ A. Kourkoumeli-Charalampidi, ${ }^{68 a, 68 b}$ C. Kourkoumelis, ${ }^{9}$ E. Kourlitis, ${ }^{146}$ V. Kouskoura, ${ }^{29}$ A. B. Kowalewska, ${ }^{82}$ R. Kowalewski, ${ }^{173}$ T. Z. Kowalski, ${ }^{81 a}$ C. Kozakai, ${ }^{160}$ W. Kozanecki, ${ }^{142}$ A. S. Kozhin, ${ }^{121}$ V. A. Kramarenko, ${ }^{111}$ G. Kramberger, ${ }^{89}$ D. Krasnopevtsev, ${ }^{110}$ M. W. Krasny, ${ }^{133}$ A. Krasznahorkay, ${ }^{35}$ D. Krauss, ${ }^{113}$ J. A. Kremer, ${ }^{81 a}$ J. Kretzschmar, ${ }^{88}$ P. Krieger, ${ }^{164}$ K. Krizka, ${ }^{18}$ K. Kroeninger, ${ }^{45}$ H. Kroha, ${ }^{113}$ J. Kroll, ${ }^{138}$ J. Kroll, ${ }^{134}$ J. Krstic, ${ }^{16}$ U. Kruchonak,${ }^{77}$ H. Krüger, ${ }^{24}$ N. Krumnack, ${ }^{76}$ M. C. Kruse, ${ }^{47}$ T. Kubota, ${ }^{102}$ S. Kuday, ${ }^{4 b}$ J. T. Kuechler, ${ }^{179}$ S. Kuehn, ${ }^{35}$ A. Kugel, ${ }^{59 a}$ F. Kuger, ${ }^{174}$ T. Kuhl, ${ }^{44}$ V. Kukhtin, ${ }^{77}$ R. Kukla, ${ }^{99}$ Y. Kulchitsky, ${ }^{105}$ S. Kuleshov, ${ }^{14 \mathrm{~b}}$ Y. P. Kulinich, ${ }^{170}$ M. Kuna, ${ }^{56}$ T. Kunigo, ${ }^{83}$ A. Kupco, ${ }^{138}$ T. Kupfer, ${ }^{45}$ O. Kuprash, ${ }^{158}$ H. Kurashige ${ }^{80}$ L. L. Kurchaninov, ${ }^{165}$ Y. A. Kurochkin, ${ }^{105}$ M. G. Kurth, ${ }^{15 \mathrm{~d}}$ E. S. Kuwertz, ${ }^{173}$ M. Kuze, ${ }^{162}$ J. Kvita, ${ }^{127}$ T. Kwan, ${ }^{173}$ A. La Rosa, ${ }^{113}$ J. L. La Rosa Navarro, ${ }^{78 \mathrm{~d}}$ L. La Rotonda, ${ }^{40 \mathrm{~b}, 40 \mathrm{a}}$ F. La Ruffa, ${ }^{40 b, 40 \mathrm{a}}$ C. Lacasta, ${ }^{171}$ F. Lacava, ${ }^{70 a}, 70 \mathrm{~b}$ J. Lacey, ${ }^{44}$ D. P. J. Lack, ${ }^{98}$ H. Lacker, ${ }^{19}$ D. Lacour, ${ }^{133}$ E. Ladygin, ${ }^{77}$ R. Lafaye, ${ }^{5}$ B. Laforge, ${ }^{133}$ T. Lagouri, ${ }^{32 c}$ S. Lai, ${ }^{51}$ S. Lammers,${ }^{63}$ W. Lampl, ${ }^{7}$ E. Lançon, ${ }^{29}$ U. Landgraf,${ }^{50}$ M. P. J. Landon, ${ }^{90}$ M. C. Lanfermann, ${ }^{52}$ V. S. Lang, ${ }^{44}$ J. C. Lange, ${ }^{14}$ R. J. Langenberg, ${ }^{35}$ A. J. Lankford, ${ }^{168}$ F. Lanni, ${ }^{29}$ K. Lantzsch, ${ }^{24}$ A. Lanza, ${ }^{68 a}$ A. Lapertosa, ${ }^{53 b, 53 a}$ S. Laplace, ${ }^{133}$ J. F. Laporte, ${ }^{142}$ T. Lari, ${ }^{66 a}$ F. Lasagni Manghi, ${ }^{23 b, 23 a}$ M. Lassnig, ${ }^{35}$ T. S. Lau, ${ }^{61 a}$ A. Laudrain, ${ }^{129}$ A. T. Law, ${ }^{143}$ P. Laycock, ${ }^{88}$ M. Lazzaroni, ${ }^{66,66 \mathrm{~b}}$ B. Le, ${ }^{102}$ O. Le Dortz, ${ }^{133}$ E. Le Guirriec, ${ }^{99}$ E. P. Le Quilleuc, ${ }^{142}$ M. LeBlanc, ${ }^{7}$ T. LeCompte, ${ }^{6}$ F. Ledroit-Guillon, ${ }^{56}$ C. A. Lee, ${ }^{29}$ G. R. Lee, ${ }^{144 a}$ L. Lee,${ }^{57}$ S. C. Lee, ${ }^{155}$ B. Lefebvre, ${ }^{101}$ M. Lefebvre, ${ }^{173}$ F. Legger, ${ }^{112}$ C. Leggett, ${ }^{18}$ G. Lehmann Miotto, ${ }^{35}$ W. A. Leight ${ }^{44}$ A. Leisos, ${ }^{159, i i}$ M. A. L. Leite, ${ }^{78 d}$ R. Leitner, ${ }^{140}$ D. Lellouch, ${ }^{177}$ B. Lemmer, ${ }^{51}$ K. J. C. Leney, ${ }^{92}$ T. Lenz,${ }^{24}$ B. Lenzi, ${ }^{35}$ R. Leone, ${ }^{7}$ S. Leone, ${ }^{69 a}$ C. Leonidopoulos, ${ }^{48}$ G. Lerner,${ }^{153}$ C. Leroy, ${ }^{107}$ R. Les, ${ }^{164}$ A. A. J. Lesage, ${ }^{142}$ C. G. Lester, ${ }^{31}$ M. Levchenko, ${ }^{135}$ J. Levêque, ${ }^{5}$ D. Levin, ${ }^{103}$ L. J. Levinson, ${ }^{177}$ D. Lewis, ${ }^{90}$

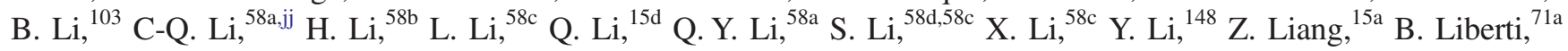
A. Liblong, ${ }^{164}$ K. Lie, ${ }^{61 \mathrm{c}}$ S. Liem, ${ }^{118}$ A. Limosani, ${ }^{154}$ C. Y. Lin, ${ }^{31}$ K. Lin, ${ }^{104}$ T. H. Lin, ${ }^{97}$ R. A. Linck, ${ }^{63}$ B. E. Lindquist,${ }^{152}$ A. L. Lionti, ${ }^{52}$ E. Lipeles, ${ }^{134}$ A. Lipniacka, ${ }^{17}$ M. Lisovyi, ${ }^{59 b}$ T. M. Liss, ${ }^{170, k k}$ A. Lister, ${ }^{172}$ A. M. Litke, ${ }^{143}$ J. D. Little, ${ }^{8}$ 
B. Liu, ${ }^{76}$ B. L. Liu, ${ }^{6}$ H. B. Liu, ${ }^{29}$ H. Liu, ${ }^{103}$ J. B. Liu,${ }^{58 a}$ J. K. K. Liu, ${ }^{132}$ K. Liu, ${ }^{133}$ M. Liu, ${ }^{58 a}$ P. Liu, ${ }^{18}$ Y. Liu, ${ }^{15 a}$ Y. L. Liu, ${ }^{58 a}$ Y. W. Liu, ${ }^{58 \mathrm{a}}$ M. Livan, ${ }^{68 \mathrm{a}, 68 \mathrm{~b}}$ A. Lleres,${ }^{56}$ J. Llorente Merino, ${ }^{15 \mathrm{a}}$ S. L. Lloyd, ${ }^{90}$ C. Y. Lo ${ }^{61 \mathrm{~b}}$ F. Lo Sterzo,${ }^{41}$ E. M. Lobodzinska, ${ }^{44}$ P. Loch, ${ }^{7}$ F. K. Loebinger, ${ }^{98}$ K. M. Loew, ${ }^{26}$ T. Lohse, ${ }^{19}$ K. Lohwasser,${ }^{146}$ M. Lokajicek, ${ }^{138}$ B. A. Long, ${ }^{25}$ J. D. Long, ${ }^{170}$ R. E. Long, ${ }^{87}$ L. Longo, ${ }^{65 a, 65 b}$ K. A. Looper ${ }^{123}$ J. A. Lopez, ${ }^{144 b}$ I. Lopez Paz, ${ }^{14}$ A. Lopez Solis, ${ }^{133}$ J. Lorenz, ${ }^{112}$ N. Lorenzo Martinez, ${ }^{5}$ M. Losada, ${ }^{22}$ P. J. Lösel,${ }^{112}$ A. Lösle, ${ }^{50}$ X. Lou, ${ }^{44}$ X. Lou, ${ }^{15 a}$ A. Lounis, ${ }^{129}$ J. Love, ${ }^{6}$ P. A. Love, ${ }^{87}$ J. J. Lozano Bahilo, ${ }^{171}$ H. Lu, ${ }^{61 a}$ N. Lu, ${ }^{103}$ Y. J. Lu, ${ }^{62}$ H. J. Lubatti, ${ }^{145}$ C. Luci, ${ }^{70 a, 70 b}$ A. Lucotte ${ }^{56}$ C. Luedtke ${ }^{50}$ F. Luehring, ${ }^{63}$ I. Luise, ${ }^{133}$ W. Lukas, ${ }^{74}$ L. Luminari, ${ }^{70 a}$ B. Lund-Jensen, ${ }^{151}$ M. S. Lutz, ${ }^{100}$ P. M. Luzi, ${ }^{133}$ D. Lynn, ${ }^{29}$ R. Lysak, ${ }^{138}$ E. Lytken, ${ }^{94}$ F. Lyu, ${ }^{15 a}$ V. Lyubushkin, ${ }^{77}$ H. Ma, ${ }^{29}$ L. L. Ma ${ }^{58 b}$ Y. Ma, ${ }^{58 b}$ G. Maccarrone, ${ }^{49}$ A. Macchiolo, ${ }^{113}$ C. M. Macdonald, ${ }^{146}$ J. Machado Miguens, ${ }^{134,137 b}$ D. Madaffari, ${ }^{171}$ R. Madar, ${ }^{37}$ W. F. Mader, ${ }^{46}$ A. Madsen, ${ }^{44}$ N. Madysa, ${ }^{46}$ J. Maeda, ${ }^{80}$ S. Maeland, ${ }^{17}$ T. Maeno, ${ }^{29}$ A. S. Maevskiy, ${ }^{11}$ V. Magerl,${ }^{50}$ C. Maidantchik, ${ }^{78 b}$ T. Maier, ${ }^{12}$ A. Maio, ${ }^{137 a, 137 b, 137 d}$ O. Majersky, ${ }^{28 a}$ S. Majewski, ${ }^{128}$ Y. Makida, ${ }^{79}$ N. Makovec, ${ }^{129}$ B. Malaescu, ${ }^{133}$ Pa. Malecki, ${ }^{82}$ V. P. Maleev, ${ }^{135}$ F. Malek, ${ }^{56}$ U. Mallik, ${ }^{75}$ D. Malon, ${ }^{6}$ C. Malone, ${ }^{31}$ S. Maltezos, ${ }^{10}$ S. Malyukov, ${ }^{35}$ J. Mamuzic, ${ }^{171}$ G. Mancini, ${ }^{49}$ I. Mandić, ${ }^{89}$ J. Maneira, ${ }^{137 a}$ L. Manhaes de Andrade Filho, ${ }^{78 a}$ J. Manjarres Ramos, ${ }^{46}$ K. H. Mankinen, ${ }^{44}$ A. Mann, ${ }^{112}$ A. Manousos,${ }^{74}$ B. Mansoulie, ${ }^{142}$ J. D. Mansour, ${ }^{15 a}$ M. Mantoani, ${ }^{51}$ S. Manzoni, ${ }^{66 a, 66 b}$ G. Marceca, ${ }^{30}$ L. March, ${ }^{52}$ L. Marchese, ${ }^{132}$ G. Marchiori, ${ }^{133}$ M. Marcisovsky, ${ }^{138}$ C. A. Marin Tobon, ${ }^{35}$ M. Marjanovic, ${ }^{37}$ D. E. Marley, ${ }^{103}$ F. Marroquim, ${ }^{78 b}$ Z. Marshall, ${ }^{18}$ M. U. F. Martensson, ${ }^{169}$ S. Marti-Garcia, ${ }^{171}$ C. B. Martin, ${ }^{123}$ T. A. Martin, ${ }^{175}$ V. J. Martin, ${ }^{48}$ B. Martin dit Latour, ${ }^{17}$ M. Martinez, ${ }^{14, x}$ V. I. Martinez Outschoorn, ${ }^{100}$ S. Martin-Haugh, ${ }^{141}$ V. S. Martoiu, ${ }^{27 b}$ A. C. Martyniuk, ${ }^{92}$ A. Marzin, ${ }^{35}$ L. Masetti, ${ }^{97}$ T. Mashimo, ${ }^{160}$ R. Mashinistov, ${ }^{108}$ J. Masik, ${ }^{98}$ A. L. Maslennikov, ${ }^{120 b, 120 \mathrm{a}}$ L. H. Mason, ${ }^{102}$ L. Massa, ${ }^{71 \mathrm{a}, 71 \mathrm{~b}}$ P. Mastrandrea, ${ }^{5}$ A. Mastroberardino, ${ }^{40 \mathrm{~b}, 40 \mathrm{a}}$ T. Masubuchi, ${ }^{160}$ P. Mättig, ${ }^{179}$ J. Maurer, ${ }^{27 b}$ B. Maček, ${ }^{89}$ S. J. Maxfield, ${ }^{88}$ D. A. Maximov, ${ }^{120 b, 120 a}$ R. Mazini,${ }^{155}$ I. Maznas, ${ }^{159}$ S. M. Mazza, ${ }^{143}$ N. C. Mc Fadden, ${ }^{116}$ G. Mc Goldrick, ${ }^{164}$ S. P. Mc Kee, ${ }^{103}$ A. McCarn, ${ }^{103}$ T. G. McCarthy, ${ }^{113}$ L. I. McClymont, ${ }^{92}$ E. F. McDonald, ${ }^{102}$ J. A. Mcfayden, ${ }^{35}$ G. Mchedlidze,${ }^{51}$ M. A. McKay, ${ }^{41}$ K. D. McLean, ${ }^{173}$ S. J. McMahon, ${ }^{141}$ P. C. McNamara, ${ }^{102}$ C. J. McNicol, ${ }^{175}$ R. A. McPherson, ${ }^{173, m}$ J. E. Mdhluli, ${ }^{32 c}$ Z. A. Meadows, ${ }^{100}$ S. Meehan, ${ }^{145}$ T. M. Megy, ${ }^{50}$ S. Mehlhase, ${ }^{112}$ A. Mehta, ${ }^{88}$ T. Meideck,${ }^{56}$ B. Meirose,${ }^{42}$ D. Melini, ${ }^{171,11}$ B. R. Mellado Garcia, ${ }^{32 \mathrm{c}}$ J. D. Mellenthin, ${ }^{51}$ M. Melo, ${ }^{28 a}$ F. Meloni,${ }^{20}$ A. Melzer, ${ }^{24}$ S. B. Menary, ${ }^{98}$

E. D. Mendes Gouveia, ${ }^{137 a}$ L. Meng, ${ }^{88}$ X. T. Meng, ${ }^{103}$ A. Mengarelli, ${ }^{23 b, 23 a}$ S. Menke, ${ }^{113}$ E. Meoni, ${ }^{40 b, 40 a}$ S. Mergelmeyer, ${ }^{19}$ C. Merlassino, ${ }^{20}$ P. Mermod, ${ }^{52}$ L. Merola,${ }^{67,67 b}$ C. Meroni, ${ }^{66 a}$ F. S. Merritt, ${ }^{36}$ A. Messina, ${ }^{70 a, 70 b}$ J. Metcalfe,${ }^{6}$ A. S. Mete, ${ }^{168}$ C. Meyer, ${ }^{134}$ J. Meyer, ${ }^{157}$ J-P. Meyer ${ }^{142}$ H. Meyer Zu Theenhausen, ${ }^{59 a}$ F. Miano, ${ }^{153}$ R. P. Middleton, ${ }^{141}$ L. Mijović, ${ }^{48}$ G. Mikenberg, ${ }^{177}$ M. Mikestikova, ${ }^{138}$ M. Mikuž, ${ }^{89}$ M. Milesi, ${ }^{102}$ A. Milic, ${ }^{164}$ D. A. Millar, ${ }^{90}$ D. W. Miller, ${ }^{36}$ A. Milov, ${ }^{177}$ D. A. Milstead, ${ }^{43,43 b}$ A. A. Minaenko, ${ }^{121}$ I. A. Minashvili, ${ }^{156 b}$ A. I. Mincer, ${ }^{122}$ B. Mindur ${ }^{81 a}$ M. Mineev, ${ }^{77}$ Y. Minegishi, ${ }^{160}$ Y. Ming, ${ }^{178}$ L. M. Mir, ${ }^{14}$ A. Mirto, ${ }^{65 a, 65 b}$ K. P. Mistry, ${ }^{134}$ T. Mitani, ${ }^{176}$ J. Mitrevski, ${ }^{112}$ V. A. Mitsou, ${ }^{171}$ A. Miucci, ${ }^{20}$ P. S. Miyagawa ${ }^{146}$ A. Mizukami ${ }^{79}$ J. U. Mjörnmark,${ }^{94}$ T. Mkrtchyan, ${ }^{181}$ M. Mlynarikova, ${ }^{140}$ T. Moa, ${ }^{43 a, 43 b}$ K. Mochizuki, ${ }^{107}$ P. Mogg, ${ }^{50}$ S. Mohapatra, ${ }^{38}$ S. Molander, ${ }^{43 a, 43 b}$ R. Moles-Valls, ${ }^{24}$ M. C. Mondragon, ${ }^{104}$ K. Mönig, ${ }^{44}$ J. Monk, ${ }^{39}$ E. Monnier, ${ }^{99}$ A. Montalbano, ${ }^{149}$ J. Montejo Berlingen, ${ }^{35}$ F. Monticelli, ${ }^{86}$ S. Monzani ${ }^{66 a}$ R. W. Moore, ${ }^{3}$ N. Morange, ${ }^{129}$ D. Moreno, ${ }^{22}$ M. Moreno Llácer, ${ }^{35}$ P. Morettini, ${ }^{53 b}$ M. Morgenstern, ${ }^{118}$ S. Morgenstern, ${ }^{35}$ D. Mori, ${ }^{149}$ T. Mori, ${ }^{160}$ M. Morii, ${ }^{57}$ M. Morinaga, ${ }^{176}$ V. Morisbak, ${ }^{131}$ A. K. Morley ${ }^{35}$ G. Mornacchi, ${ }^{35}$ A. P. Morris, ${ }^{92}$ J. D. Morris,${ }^{90}$ L. Morvaj, ${ }^{152}$ P. Moschovakos, ${ }^{10}$ M. Mosidze, ${ }^{156 \mathrm{~b}}$ H. J. Moss, ${ }^{146}$ J. Moss, ${ }^{150, \mathrm{~mm}}$ K. Motohashi, ${ }^{162}$ R. Mount, ${ }^{150}$ E. Mountricha ${ }^{35}$ E. J. W. Moyse, ${ }^{100}$ S. Muanza, ${ }^{99}$ F. Mueller, ${ }^{113}$ J. Mueller, ${ }^{136}$ R. S. P. Mueller, ${ }^{12}$ D. Muenstermann, ${ }^{87}$ P. Mullen, ${ }^{55}$ G. A. Mullier, ${ }^{20}$ F. J. Munoz Sanchez, ${ }^{98}$ P. Murin,${ }^{28 b}$ W. J. Murray, ${ }^{175,141}$ A. Murrone,${ }^{66,66 b}$ M. Muškinja, ${ }^{89}$ C. Mwewa, ${ }^{32 a}$ A. G. Myagkov, ${ }^{121, \mathrm{nn}}$ J. Myers, ${ }^{128}$ M. Myska, ${ }^{139}$ B.P. Nachman, ${ }^{18}$ O. Nackenhorst,${ }^{45}$ K. Nagai, ${ }^{132}$ K. Nagano, ${ }^{79}$ Y. Nagasaka, ${ }^{60}$ K. Nagata, ${ }^{166}$ M. Nagel,${ }^{50}$ E. Nagy, ${ }^{99}$ A. M. Nairz, ${ }^{35}$ Y. Nakahama, ${ }^{15}$ K. Nakamura, ${ }^{79}$ T. Nakamura, ${ }^{160}$ I. Nakano, ${ }^{124}$ H. Nanjo, ${ }^{130}$ F. Napolitano, ${ }^{59 a}$ R. F. Naranjo Garcia,${ }^{44}$ R. Narayan, ${ }^{11}$ D. I. Narrias Villar, ${ }^{59 a}$ I. Naryshkin, ${ }^{135}$ T. Naumann, ${ }^{44}$ G. Navarro, ${ }^{22}$ R. Nayyar, ${ }^{7}$ H. A. Neal, ${ }^{103, a}$ P. Y. Nechaeva, ${ }^{108}$ T. J. Neep, ${ }^{142}$ A. Negri, ${ }^{68,68 b}$ M. Negrini, ${ }^{23 b}$ S. Nektarijevic, ${ }^{117}$ C. Nellist, ${ }^{51}$ M. E. Nelson, ${ }^{132}$ S. Nemecek, ${ }^{138}$ P. Nemethy, ${ }^{122}$ M. Nessi, ${ }^{35,00}$ M. S. Neubauer, ${ }^{170}$ M. Neumann, ${ }^{179}$ P. R. Newman, ${ }^{21}$ T. Y. Ng, ${ }^{61 \mathrm{c}}$ Y. S. Ng, ${ }^{19}$ H. D. N. Nguyen, ${ }^{99}$ T. Nguyen Manh, ${ }^{107}$ E. Nibigira, ${ }^{37}$ R. B. Nickerson, ${ }^{132}$ R. Nicolaidou, ${ }^{142}$ J. Nielsen, ${ }^{143}$ N. Nikiforou, ${ }^{11}$ V. Nikolaenko, ${ }^{121, n n}$ I. Nikolic-Audit, ${ }^{133}$ K. Nikolopoulos ${ }^{21}$ P. Nilsson, ${ }^{29}$ Y. Ninomiya,${ }^{79}$ A. Nisati, ${ }^{70 a}$ N. Nishu, ${ }^{58 c}$ R. Nisius, ${ }^{113}$ I. Nitsche, ${ }^{45}$ T. Nitta, ${ }^{176}$ T. Nobe,${ }^{160}$ Y. Noguchi, ${ }^{83}$ M. Nomachi, ${ }^{130}$ I. Nomidis, ${ }^{133}$ M. A. Nomura,${ }^{29}$ T. Nooney, ${ }^{90}$ M. Nordberg,${ }^{35}$ N. Norjoharuddeen, ${ }^{132}$ T. Novak, ${ }^{89}$ O. Novgorodova, ${ }^{46}$ R. Novotny, ${ }^{139}$ M. Nozaki, ${ }^{79}$ L. Nozka, ${ }^{127}$ K. Ntekas, ${ }^{168}$ E. Nurse, ${ }^{92}$ F. Nuti, ${ }^{102}$ 
F. G. Oakham, ${ }^{33, e}$ H. Oberlack, ${ }^{113}$ T. Obermann, ${ }^{24}$ J. Ocariz, ${ }^{133}$ A. Ochi, ${ }^{80}$ I. Ochoa, ${ }^{38}$ J. P. Ochoa-Ricoux, ${ }^{144 a}$ K. O'Connor, ${ }^{26}$ S. Oda, ${ }^{85}$ S. Odaka, ${ }^{79}$ A. Oh, ${ }^{98}$ S. H. Oh, ${ }^{47}$ C. C. Ohm, ${ }^{151}$ H. Oide,${ }^{53 b, 53 a}$ H. Okawa, ${ }^{166}$ Y. Okazaki, ${ }^{83}$ Y. Okumura, ${ }^{160}$ T. Okuyama, ${ }^{79}$ A. Olariu, ${ }^{27 b}$ L. F. Oleiro Seabra, ${ }^{137 a}$ S. A. Olivares Pino, ${ }^{144 a}$ D. Oliveira Damazio, ${ }^{29}$ J. L. Oliver, ${ }^{1}$ M. J. R. Olsson, ${ }^{36}$ A. Olszewski, ${ }^{82}$ J. Olszowska, ${ }^{82}$ D. C. O’Neil, ${ }^{149}$ A. Onofre, ${ }^{137 a, 137 e}$ K. Onogi, ${ }^{115}$ P. U. E. Onyisi, ${ }^{11}$ H. Oppen, ${ }^{131}$ M. J. Oreglia, ${ }^{36}$ Y. Oren, ${ }^{158}$ D. Orestano, ${ }^{72 a, 72 b}$ E. C. Orgill, ${ }^{98}$ N. Orlando, ${ }^{61 b}$ A. A. O'Rourke, ${ }^{44}$ R. S. Orr, ${ }^{164}$ B. Osculati, ${ }^{53 b, 53 a, a}$ V. O’Shea, ${ }^{55}$ R. Ospanov, ${ }^{58 a}$ G. Otero y Garzon, ${ }^{30}$ H. Otono, ${ }^{85}$ M. Ouchrif, ${ }^{34 \mathrm{~d}}$ F. Ould-Saada, ${ }^{131}$ A. Ouraou, ${ }^{142}$ Q. Ouyang, ${ }^{15 a}$ M. Owen, ${ }^{55}$ R. E. Owen, ${ }^{21}$ V. E. Ozcan, ${ }^{12 c}$ N. Ozturk, ${ }^{8}$ J. Pacalt, ${ }^{127}$ H. A. Pacey, ${ }^{31}$ K. Pachal, ${ }^{149}$ A. Pacheco Pages, ${ }^{14}$ L. Pacheco Rodriguez, ${ }^{142}$ C. Padilla Aranda, ${ }^{14}$ S. Pagan Griso, ${ }^{18}$ M. Paganini, ${ }^{180}$ G. Palacino, ${ }^{63}$ S. Palazzo, ${ }^{40 b, 40 a}$ S. Palestini, ${ }^{35}$ M. Palka, ${ }^{81 b}$ D. Pallin, ${ }^{37}$ I. Panagoulias, ${ }^{10}$ C. E. Pandini, ${ }^{35}$ J. G. Panduro Vazquez, ${ }^{91}$ P. Pani, ${ }^{35}$ G. Panizzo, ${ }^{64 a, 64 c}$ L. Paolozzi, ${ }^{52}$ T. D. Papadopoulou, ${ }^{10}$ K. Papageorgiou, ${ }^{9, t}$ A. Paramonov, ${ }^{6}$ D. Paredes Hernandez, ${ }^{61 \mathrm{~b}}$ B. Parida, ${ }^{58 \mathrm{c}}$ A. J. Parker, ${ }^{87}$ K. A. Parker, ${ }^{44}$ M. A. Parker, ${ }^{31}$ F. Parodi, ${ }^{53 b, 53 a}$ J. A. Parsons, ${ }^{38}$ U. Parzefall, ${ }^{50}$ V. R. Pascuzzi, ${ }^{164}$ J. M. P. Pasner, ${ }^{143}$ E. Pasqualucci, ${ }^{70 a}$ S. Passaggio, ${ }^{53 b}$ F. Pastore, ${ }^{91}$ P. Pasuwan, ${ }^{43 a, 43 b}$ S. Pataraia, ${ }^{97}$ J. R. Pater, ${ }^{98}$ A. Pathak, ${ }^{178, f}$ T. Pauly, ${ }^{35}$ B. Pearson, ${ }^{113}$ M. Pedersen, ${ }^{131}$ L. Pedraza Diaz, ${ }^{117}$ S. Pedraza Lopez, ${ }^{171}$ R. Pedro, ${ }^{137 a, 137 b}$ S. V. Peleganchuk, ${ }^{120 b, 120 a}$ O. Penc, ${ }^{138}$ C. Peng, ${ }^{15 d}$ H. Peng, ${ }^{58 a}$ B. S. Peralva, ${ }^{78 a}$ M. M. Perego, ${ }^{142}$ A. P. Pereira Peixoto, ${ }^{137 a}$ D. V. Perepelitsa, ${ }^{29}$ F. Peri, ${ }^{19}$ L. Perini, ${ }^{66 a, 66 b}$ H. Pernegger, ${ }^{35}$ S. Perrella, ${ }^{67 a, 67 b}$ V. D. Peshekhonov, ${ }^{77, a}$ K. Peters, ${ }^{44}$ R. F. Y. Peters, ${ }^{98}$ B. A. Petersen, ${ }^{35}$ T. C. Petersen, ${ }^{39}$ E. Petit, ${ }^{56}$ A. Petridis, ${ }^{1}$ C. Petridou, ${ }^{159}$ P. Petroff, ${ }^{129}$ E. Petrolo, ${ }^{70 a}$ M. Petrov, ${ }^{132}$ F. Petrucci, ${ }^{72 a, 72 b}$ M. Pettee, ${ }^{180}$ N. E. Pettersson, ${ }^{100}$ A. Peyaud, ${ }^{142}$ R. Pezoa, ${ }^{144 b}$ T. Pham, ${ }^{102}$ F. H. Phillips, ${ }^{104}$ P. W. Phillips, ${ }^{141}$ G. Piacquadio, ${ }^{152}$ E. Pianori, ${ }^{18}$ A. Picazio, ${ }^{100}$ M. A. Pickering, ${ }^{132}$ R. Piegaia, ${ }^{30}$ J. E. Pilcher, ${ }^{36}$ A. D. Pilkington, ${ }^{98}$ M. Pinamonti, ${ }^{71 a, 71 b}$ J. L. Pinfold, ${ }^{3}$ M. Pitt, ${ }^{177}$ M.-A. Pleier, ${ }^{29}$ V. Pleskot, ${ }^{140}$ E. Plotnikova, ${ }^{77}$ D. Pluth, ${ }^{76}$ P. Podberezko, ${ }^{120 b, 120 a}$ R. Poettgen, ${ }^{94}$ R. Poggi, ${ }^{52}$ L. Poggioli, ${ }^{129}$ I. Pogrebnyak, ${ }^{104}$ D. Pohl, ${ }^{24}$ I. Pokharel, ${ }^{51}$ G. Polesello, ${ }^{68 a}$ A. Poley, ${ }^{44}$ A. Policicchio, ${ }^{40 b, 40 a}$ R. Polifka, ${ }^{35}$ A. Polini, ${ }^{23 b}$ C. S. Pollard, ${ }^{44}$ V. Polychronakos, ${ }^{29}$ D. Ponomarenko, ${ }^{110}$ L. Pontecorvo, ${ }^{35}$ G. A. Popeneciu, ${ }^{27 d}$ D. M. Portillo Quintero, ${ }^{133}$ S. Pospisil, ${ }^{139}$ K. Potamianos, ${ }^{44}$ I. N. Potrap, ${ }^{77}$ C. J. Potter, ${ }^{31}$ H. Potti, ${ }^{11}$ T. Poulsen, ${ }^{94}$ J. Poveda, ${ }^{35}$ T. D. Powell, ${ }^{146}$ M. E. Pozo Astigarraga, ${ }^{35}$ P. Pralavorio, ${ }^{99}$ S. Prell, ${ }^{76}$ D. Price, ${ }^{98}$ M. Primavera, ${ }^{65 a}$ S. Prince, ${ }^{101}$ N. Proklova, ${ }^{110}$ K. Prokofiev, ${ }^{61 \mathrm{c}}$ F. Prokoshin, ${ }^{144 \mathrm{~b}}$ S. Protopopescu, ${ }^{29}$ J. Proudfoot, ${ }^{6}$ M. Przybycien, ${ }^{81 \mathrm{a}}$ A. Puri, ${ }^{170}$ P. Puzo, ${ }^{129}$ J. Qian, ${ }^{103}$ Y. Qin, ${ }^{98}$ A. Quadt, ${ }^{51}$ M. Queitsch-Maitland, ${ }^{44}$ A. Qureshi, ${ }^{1}$ P. Rados, ${ }^{102}$ F. Ragusa, ${ }^{66,66 b}$ G. Rahal, ${ }^{95}$ J. A. Raine, ${ }^{98}$ S. Rajagopalan, ${ }^{29}$ A. Ramirez Morales, ${ }^{90}$ T. Rashid, ${ }^{129}$ S. Raspopov, ${ }^{5}$ M. G. Ratti, ${ }^{66 a, 66 b}$ D. M. Rauch, ${ }^{44}$ F. Rauscher, ${ }^{112}$ S. Rave, ${ }^{97}$ B. Ravina, ${ }^{146}$ I. Ravinovich, ${ }^{177}$ J. H. Rawling, ${ }^{98}$ M. Raymond, ${ }^{35}$ A. L. Read, ${ }^{131}$ N. P. Readioff, ${ }^{56}$ M. Reale, ${ }^{65 a, 65 b}$ D. M. Rebuzzi, ${ }^{68 a, 68 b}$ A. Redelbach, ${ }^{174}$ G. Redlinger, ${ }^{29}$ R. Reece, ${ }^{143}$ R. G. Reed, ${ }^{32 c}$ K. Reeves, ${ }^{42}$ L. Rehnisch, ${ }^{19}$ J. Reichert, ${ }^{134}$ A. Reiss, ${ }^{97}$ C. Rembser, ${ }^{35}$ H. Ren, ${ }^{15 d}$ M. Rescigno, ${ }^{70 a}$ S. Resconi, ${ }^{66 a}$ E. D. Resseguie, ${ }^{134}$ S. Rettie, ${ }^{172}$ E. Reynolds, ${ }^{21}$ O. L. Rezanova, ${ }^{120 b, 120 \mathrm{a}}$ P. Reznicek, ${ }^{140}$ R. Richter, ${ }^{113}$ S. Richter, ${ }^{92}$ E. Richter-Was, ${ }^{81 b}$ O. Ricken, ${ }^{24}$ M. Ridel, ${ }^{133}$ P. Rieck, ${ }^{113}$ C. J. Riegel, ${ }^{179}$ O. Rifki, ${ }^{44}$ M. Rijssenbeek, ${ }^{152}$ A. Rimoldi, ${ }^{68 a, 68 b}$ M. Rimoldi, ${ }^{20}$ L. Rinaldi, $^{23 b}$ G. Ripellino, ${ }^{151}$ B. Ristić, ${ }^{87}$ E. Ritsch, ${ }^{35}$ I. Riu, ${ }^{14}$ J. C. Rivera Vergara, ${ }^{144 a}{ }^{\text {F. Rizatdinova, }}{ }^{126}$ E. Rizvi, ${ }^{90}$ C. Rizzi, ${ }^{14}$ R. T. Roberts, ${ }^{98}$ S. H. Robertson, ${ }^{101, m}$ A. Robichaud-Veronneau, ${ }^{101}$ D. Robinson, ${ }^{31}$ J. E. M. Robinson, ${ }^{44}$ A. Robson, ${ }^{55}$ E. Rocco, ${ }^{97}$ C. Roda, ${ }^{69 a, 69 b}$ Y. Rodina,${ }^{99}$ S. Rodriguez Bosca, ${ }^{171}$ A. Rodriguez Perez, ${ }^{14}$ D. Rodriguez Rodriguez, ${ }^{171}$ A. M. Rodríguez Vera, ${ }^{165 b}$ S. Roe, ${ }^{35}$ C. S. Rogan, ${ }^{57}$ O. Røhne, ${ }^{131}$ R. Röhrig, ${ }^{113}$ C. P. A. Roland, ${ }^{63}$ J. Roloff, $^{57}$ A. Romaniouk, ${ }^{110}$ M. Romano, ${ }^{23 b, 23 a}$ N. Rompotis, ${ }^{88}$ M. Ronzani, ${ }^{122}$ L. Roos, ${ }^{133}$ S. Rosati, ${ }^{70 a}$ K. Rosbach, ${ }^{50}$ P. Rose, ${ }^{143}$ N-A. Rosien, ${ }^{51}$ E. Rossi, ${ }^{67 a, 67 b}$ L. P. Rossi, ${ }^{53 b}$ L. Rossini, ${ }^{66 a, 66 b}$ J. H. N. Rosten, ${ }^{31}$ R. Rosten, ${ }^{14}$ M. Rotaru, ${ }^{27 b}$ J. Rothberg, ${ }^{145}$ D. Rousseau, ${ }^{129}$ D. Roy, ${ }^{32 c}$ A. Rozanov, ${ }^{99}$ Y. Rozen, ${ }^{157}$ X. Ruan, ${ }^{32 c}$ F. Rubbo, ${ }^{150}$ F. Rühr, ${ }^{50}$ A. Ruiz-Martinez, ${ }^{33}$ Z. Rurikova, ${ }^{50}$ N. A. Rusakovich, ${ }^{77}$ H. L. Russell, ${ }^{101}$ J. P. Rutherfoord, ${ }^{7}$ N. Ruthmann, ${ }^{35}$ E. M. Rüttinger, ${ }^{44, p p}$ Y. F. Ryabov, ${ }^{135}$ M. Rybar, ${ }^{170}$ G. Rybkin, ${ }^{129}$ S. Ryu, ${ }^{6}$ A. Ryzhov, ${ }^{121}$ G. F. Rzehorz, ${ }^{51}$ P. Sabatini, ${ }^{51}$ G. Sabato, ${ }^{118}$ S. Sacerdoti, ${ }^{129}$ H. F-W. Sadrozinski, ${ }^{143}$ R. Sadykov, ${ }^{77}$ F. Safai Tehrani, ${ }^{70 a}$ P. Saha, ${ }^{119}$ M. Sahinsoy, ${ }^{59 a}$ A. Sahu, ${ }^{179}$ M. Saimpert, ${ }^{44}$ M. Saito, ${ }^{160}$ T. Saito, ${ }^{160}$ H. Sakamoto, ${ }^{160}$ A. Sakharov, ${ }^{122, h h}$ D. Salamani, ${ }^{52}$ G. Salamanna, ${ }^{72 a, 72 b}$ J. E. Salazar Loyola, ${ }^{14 b}$ D. Salek, ${ }^{118}$ P. H. Sales De Bruin, ${ }^{169}$ D. Salihagic, ${ }^{113}$ A. Salnikov, ${ }^{150}$ J. Salt, ${ }^{171}$ D. Salvatore, ${ }^{40 b, 40 a}$ F. Salvatore, ${ }^{153}$ A. Salvucci, ${ }^{61 \mathrm{a}, 61 \mathrm{~b}, 61 \mathrm{c}}$ A. Salzburger, ${ }^{35}$ D. Sammel, ${ }^{50}$ D. Sampsonidis, ${ }^{159}$ D. Sampsonidou, ${ }^{159}$ J. Sánchez, ${ }^{171}$ A. Sanchez Pineda, ${ }^{64 a, 64 c}$ H. Sandaker, ${ }^{131}$ C. O. Sander, ${ }^{44}$ M. Sandhoff, ${ }^{179}$ C. Sandoval, ${ }^{22}$ D. P. C. Sankey, ${ }^{141}$ M. Sannino, ${ }^{53 b, 53 a}$ Y. Sano, ${ }^{115}$ A. Sansoni, ${ }^{49}$ C. Santoni, ${ }^{37}$ H. Santos, ${ }^{137 a}$ I. Santoyo Castillo, ${ }^{153}$ A. Sapronov, ${ }^{77}$ J. G. Saraiva, ${ }^{137 a, 137 d}$ O. Sasaki, ${ }^{79}$ K. Sato, ${ }^{166}$ E. Sauvan, ${ }^{5}$ P. Savard, ${ }^{164, e}$ N. Savic, ${ }^{113}$ R. Sawada, ${ }^{160}$ C. Sawyer, ${ }^{141}$ L. Sawyer, ${ }^{93, v}$ C. Sbarra, ${ }^{23 b}$ A. Sbrizzi, ${ }^{23 b, 23 a}$ T. Scanlon, ${ }^{92}$ J. Schaarschmidt, ${ }^{145}$ P. Schacht, ${ }^{113}$ B. M. Schachtner, ${ }^{112}$ 
D. Schaefer, ${ }^{36}$ L. Schaefer, ${ }^{134}$ J. Schaeffer, ${ }^{97}$ S. Schaepe, ${ }^{35}$ U. Schäfer, ${ }^{97}$ A. C. Schaffer, ${ }^{129}$ D. Schaile, ${ }^{112}$ R. D. Schamberger, ${ }^{152}$ N. Scharmberg, ${ }^{98}$ V. A. Schegelsky, ${ }^{135}$ D. Scheirich, ${ }^{140}$ F. Schenck, ${ }^{19}$ M. Schernau, ${ }^{168}$ C. Schiavi, ${ }^{53 b, 53 a}$ S. Schier, ${ }^{143}$ L. K. Schildgen,${ }^{24}$ Z. M. Schillaci, ${ }^{26}$ E. J. Schioppa, ${ }^{35}$ M. Schioppa, ${ }^{40 b, 40 a}$ K. E. Schleicher, ${ }^{50}$ S. Schlenker, ${ }^{35}$ K. R. Schmidt-Sommerfeld, ${ }^{113}$ K. Schmieden, ${ }^{35}$ C. Schmitt, ${ }^{97}$ S. Schmitt, ${ }^{44}$ S. Schmitz, ${ }^{97}$ U. Schnoor, ${ }^{50}$ L. Schoeffel, ${ }^{142}$ A. Schoening, ${ }^{59 b}$ E. Schopf, ${ }^{24}$ M. Schott, ${ }^{97}$ J. F. P. Schouwenberg, ${ }^{117}$ J. Schovancova, ${ }^{35}$ S. Schramm, ${ }^{52}$ A. Schulte, ${ }^{97}$ H-C. Schultz-Coulon, ${ }^{59 a}$ M. Schumacher, ${ }^{50}$ B. A. Schumm, ${ }^{143}$ Ph. Schune, ${ }^{142}$ A. Schwartzman, ${ }^{150}$

T. A. Schwarz, ${ }^{103}$ H. Schweiger, ${ }^{98}$ Ph. Schwemling, ${ }^{142}$ R. Schwienhorst, ${ }^{104}$ A. Sciandra, ${ }^{24}$ G. Sciolla, ${ }^{26}$ M. Scornajenghi, ${ }^{40 b, 40 a}$ F. Scuri, ${ }^{69 a}$ F. Scutti, ${ }^{102}$ L. M. Scyboz, ${ }^{113}$ J. Searcy, ${ }^{103}$ C. D. Sebastiani, ${ }^{70 a, 70 b}$ P. Seema, ${ }^{24}$

S. C. Seidel, ${ }^{116}$ A. Seiden, ${ }^{143}$ T. Seiss, ${ }^{36}$ J. M. Seixas, ${ }^{78 b}$ G. Sekhniaidze, ${ }^{67 a}$ K. Sekhon, ${ }^{103}$ S. J. Sekula, ${ }^{41}$ N. Semprini-Cesari, ${ }^{23 b, 23 a}$ S. Sen, ${ }^{47}$ S. Senkin, ${ }^{37}$ C. Serfon, ${ }^{131}$ L. Serin, ${ }^{129}$ L. Serkin, ${ }^{64 a, 64 b}$ M. Sessa, ${ }^{72 a, 72 b}$ H. Severini, ${ }^{125}$ F. Sforza, ${ }^{167}$ A. Sfyrla, ${ }^{52}$ E. Shabalina, ${ }^{51}$ J. D. Shahinian, ${ }^{143}$ N. W. Shaikh,${ }^{43 a, 43 b}$ L. Y. Shan, ${ }^{15 a}$ R. Shang, ${ }^{170}$ J. T. Shank, ${ }^{25}$ M. Shapiro, ${ }^{18}$ A. S. Sharma, ${ }^{1}$ A. Sharma, ${ }^{132}$ P. B. Shatalov, ${ }^{109}$ K. Shaw, ${ }^{153}$ S. M. Shaw, ${ }^{98}$ A. Shcherbakova, ${ }^{135}$ Y. Shen, ${ }^{125}$ N. Sherafati, ${ }^{33}$ A. D. Sherman, ${ }^{25}$ P. Sherwood, ${ }^{92}$ L. Shi, ${ }^{155, q 9}$ S. Shimizu, ${ }^{80}$ C. O. Shimmin, ${ }^{180}$ M. Shimojima, ${ }^{114}$ I. P. J. Shipsey, ${ }^{132}$ S. Shirabe, ${ }^{85}$ M. Shiyakova, ${ }^{77}$ J. Shlomi, ${ }^{177}$ A. Shmeleva, ${ }^{108}$ D. Shoaleh Saadi, ${ }^{107}$ M. J. Shochet, ${ }^{36}$ S. Shojaii, ${ }^{102}$ D. R. Shope, ${ }^{125}$ S. Shrestha, ${ }^{123}$ E. Shulga, ${ }^{110}$ P. Sicho, ${ }^{138}$ A. M. Sickles, ${ }^{170}$ P. E. Sidebo, ${ }^{151}$

E. Sideras Haddad, ${ }^{32 c}$ O. Sidiropoulou, ${ }^{174}$ A. Sidoti, ${ }^{23 b, 23 a}$ F. Siegert, ${ }^{46}$ Dj. Sijacki, ${ }^{16}$ J. Silva, ${ }^{137 a}$ M. Silva Jr., ${ }^{178}$ M. V. Silva Oliveira, ${ }^{78 a}$ S. B. Silverstein, ${ }^{43 a}$ L. Simic, ${ }^{77}$ S. Simion, ${ }^{129}$ E. Simioni, ${ }^{97}$ M. Simon, ${ }^{97}$ P. Sinervo, ${ }^{164}$ N. B. Sinev, ${ }^{128}$ M. Sioli, ${ }^{23 b, 23 a}$ G. Siragusa, ${ }^{174}$ I. Siral, ${ }^{103}$ S. Yu. Sivoklokov, ${ }^{111}$ J. Sjölin, ${ }^{43 a, 43 b}$ M. B. Skinner, ${ }^{87}$ P. Skubic, ${ }^{125}$ M. Slater, ${ }^{21}$ T. Slavicek, ${ }^{139}$ M. Slawinska, ${ }^{82}$ K. Sliwa, ${ }^{167}$ R. Slovak, ${ }^{140}$ V. Smakhtin, ${ }^{177}$ B. H. Smart, ${ }^{5}$ J. Smiesko, ${ }^{28 a}$ N. Smirnov, ${ }^{110}$ S. Yu. Smirnov, ${ }^{110}$ Y. Smirnov, ${ }^{110}$ L. N. Smirnova, ${ }^{111}$ O. Smirnova, ${ }^{94}$ J. W. Smith, ${ }^{51}$ M. N. K. Smith, ${ }^{38}$ R. W. Smith, ${ }^{38}$ M. Smizanska, ${ }^{87}$ K. Smolek, ${ }^{139}$ A. A. Snesarev, ${ }^{108}$ I. M. Snyder, ${ }^{128}$ S. Snyder, ${ }^{29}$ R. Sobie, ${ }^{173, m}$ A. M. Soffa, ${ }^{168}$ A. Soffer, ${ }^{178}$ A. Søgaard ${ }^{48}$ D. A. Soh, ${ }^{155}$ G. Sokhrannyi, ${ }^{89}$ C. A. Solans Sanchez, ${ }^{35}$ M. Solar, ${ }^{139}$ E. Yu. Soldatov, ${ }^{110}$ U. Soldevila, ${ }^{171}$ A. A. Solodkov, ${ }^{121}$ A. Soloshenko, ${ }^{77}$ O. V. Solovyanov, ${ }^{121}$ V. Solovyev, ${ }^{135}$ P. Sommer, ${ }^{146}$ H. Son, ${ }^{167}$ W. Song, ${ }^{141}$

A. Sopczak, ${ }^{139}$ F. Sopkova, ${ }^{28 b}$ D. Sosa, ${ }^{59 b}$ C. L. Sotiropoulou, ${ }^{69 a, 69 b}$ S. Sottocornola, ${ }^{6 a, 68 b}$ R. Soualah, ${ }^{64 a, 64 c, r r}$ A. M. Soukharev, ${ }^{120 b, 120 a}$ D. South, ${ }^{44}$ B. C. Sowden, ${ }^{91}$ S. Spagnolo, ${ }^{65 a, 65 b}$ M. Spalla, ${ }^{113}$ M. Spangenberg, ${ }^{175}$ F. Spanò, ${ }^{91}$ D. Sperlich, ${ }^{19}$ F. Spettel, ${ }^{113}$ T. M. Spieker, ${ }^{59 a}$ R. Spighi, ${ }^{23 b}$ G. Spigo,${ }^{35}$ L. A. Spiller, ${ }^{102}$ D. P. Spiteri,${ }^{55}$ M. Spousta, ${ }^{140}$ A. Stabile, ${ }^{66 a, 66 b}$ R. Stamen, ${ }^{59 a}$ S. Stamm, ${ }^{19}$ E. Stanecka, ${ }^{82}$ R. W. Stanek, ${ }^{6}$ C. Stanescu, ${ }^{72 a}$ B. Stanislaus,${ }^{132}$ M. M. Stanitzki, ${ }^{44}$ B. Stapf, ${ }^{118}$ S. Stapnes, ${ }^{131}$ E. A. Starchenko, ${ }^{121}$ G. H. Stark,${ }^{36}$ J. Stark, ${ }^{56}$ S. H. Stark, ${ }^{39}$ P. Staroba,${ }^{138}$ P. Starovoitov, ${ }^{59 a}$ S. Stärz, ${ }^{35}$ R. Staszewski, ${ }^{82}$ M. Stegler, ${ }^{44}$ P. Steinberg, ${ }^{29}$ B. Stelzer, ${ }^{149}$ H. J. Stelzer, ${ }^{35}$ O. Stelzer-Chilton, ${ }^{165 a}$ H. Stenzel,${ }^{54}$

T. J. Stevenson, ${ }^{90}$ G. A. Stewart ${ }^{55}$ M. C. Stockton, ${ }^{128}$ G. Stoicea, ${ }^{27 b}$ P. Stolte, ${ }^{51}$ S. Stonjek, ${ }^{113}$ A. Straessner, ${ }^{46}$ J. Strandberg, ${ }^{151}$ S. Strandberg, ${ }^{43 a, 43 b}$ M. Strauss, ${ }^{125}$ P. Strizenec,${ }^{28 b}$ R. Ströhmer,${ }^{174}$ D. M. Strom, ${ }^{128}$ R. Stroynowski, ${ }^{41}$ A. Strubig ${ }^{48}$ S. A. Stucci, ${ }^{29}$ B. Stugu, ${ }^{17}$ J. Stupak, ${ }^{125}$ N. A. Styles, ${ }^{44}$ D. Su, ${ }^{150}$ J. Su, ${ }^{136}$ S. Suchek ${ }^{59 a}$ Y. Sugaya,${ }^{130}$ M. Suk, ${ }^{139}$ V. V. Sulin, ${ }^{108}$ D. M. S. Sultan, ${ }^{52}$ S. Sultansoy, ${ }^{4 \mathrm{c}}$ T. Sumida, ${ }^{83}$ S. Sun, ${ }^{103}$ X. Sun, ${ }^{3}$ K. Suruliz, ${ }^{153}$ C. J. E. Suster, ${ }^{154}$ M. R. Sutton, ${ }^{153}$ S. Suzuki, ${ }^{79}$ M. Svatos, ${ }^{138}$ M. Swiatlowski, ${ }^{36}$ S. P. Swift, ${ }^{2}$ A. Sydorenko, ${ }^{97}$ I. Sykora, ${ }^{28 a}$ T. Sykora, ${ }^{140}$ D. Ta ${ }^{97}$ K. Tackmann, ${ }^{44, s s}$ J. Taenzer, ${ }^{158}$ A. Taffard, ${ }^{168}$ R. Tafirout, ${ }^{165 a}$ E. Tahirovic, ${ }^{90}$ N. Taiblum, ${ }^{158}$ H. Takai, ${ }^{29}$ R. Takashima ${ }^{84}$ E. H. Takasugi, ${ }^{113}$ K. Takeda,${ }^{80}$ T. Takeshita, ${ }^{147}$ Y. Takubo ${ }^{79}$ M. Talby, ${ }^{99}$ A. A. Talyshev, ${ }^{120 b, 120 a}$ J. Tanaka, ${ }^{160}$ M. Tanaka, ${ }^{162}$ R. Tanaka ${ }^{129}$ R. Tanioka ${ }^{80}$ B. B. Tannenwald ${ }^{123}$ S. Tapia Araya, ${ }^{144 \mathrm{~b}}$ S. Tapprogge, ${ }^{97}$ A. Tarek Abouelfadl Mohamed, ${ }^{133}$ S. Tarem, ${ }^{157}$ G. Tarna,${ }^{27 b, q}$ G. F. Tartarelli, ${ }^{66 a}$ P. Tas, ${ }^{140}$ M. Tasevsky,${ }^{138}$ T. Tashiro, ${ }^{83}$ E. Tassi, ${ }^{40 b, 40 a}$ A. Tavares Delgado, ${ }^{137 a, 137 b}$ Y. Tayalati, ${ }^{34 \mathrm{e}}$ A. C. Taylor, ${ }^{116}$ A. J. Taylor, ${ }^{48}$ G. N. Taylor, ${ }^{102}$ P. T. E. Taylor, ${ }^{102}$ W. Taylor, ${ }^{165 b}$ A. S. Tee, ${ }^{87}$ P. Teixeira-Dias, ${ }^{91}$ D. Temple, ${ }^{149}$ H. Ten Kate,${ }^{35}$ P. K. Teng, ${ }^{155}$ J. J. Teoh,${ }^{130}$ F. Tepel, ${ }^{179}$ S. Terada, ${ }^{79}$ K. Terashi, ${ }^{160}$ J. Terron, ${ }^{96}$ S. Terzo,${ }^{14}$ M. Testa, ${ }^{49}$ R. J. Teuscher,${ }^{164, \mathrm{~m}}$ S. J. Thais, ${ }^{180}$ T. Theveneaux-Pelzer,${ }^{44}$ F. Thiele, ${ }^{39}$ J. P. Thomas, ${ }^{21}$ A. S. Thompson, ${ }^{55}$ P. D. Thompson, ${ }^{21}$ L. A. Thomsen, ${ }^{180}$ E. Thomson, ${ }^{134}$ Y. Tian, ${ }^{38}$ R. E. Ticse Torres,${ }^{51}$ V. O. Tikhomirov, ${ }^{108, t \mathrm{t}}$ Yu. A. Tikhonov, ${ }^{120 b, 120 \mathrm{a}}$ S. Timoshenko, ${ }^{110}$ P. Tipton, ${ }^{180}$ S. Tisserant, ${ }^{99}$ K. Todome, ${ }^{162}$ S. Todorova-Nova, ${ }^{5}$ S. Todt, ${ }^{46}$ J. Tojo, ${ }^{85}$ S. Tokár, ${ }^{28 a}$ K. Tokushuku, ${ }^{79}$ E. Tolley, ${ }^{123}$ K. G. Tomiwa ${ }^{32 c}$ M. Tomoto, ${ }^{115}$ L. Tompkins, ${ }^{150, \mathrm{gg}}$ K. Toms, ${ }^{116}$ B. Tong, ${ }^{57}$ P. Tornambe, ${ }^{50}$ E. Torrence, ${ }^{128}$ H. Torres, ${ }^{46}$ E. Torró Pastor, ${ }^{145}$ C. Tosciri, ${ }^{132}$ J. Toth,${ }^{9, \text {,u }}$ F. Touchard, ${ }^{99}$ D. R. Tovey, ${ }^{146}$ C. J. Treado, ${ }^{122}$ T. Trefzger, ${ }^{174}$ F. Tresoldi, ${ }^{153}$ A. Tricoli, ${ }^{29}$ I. M. Trigger, ${ }^{165 a}$ S. Trincaz-Duvoid,${ }^{133}$ M. F. Tripiana, ${ }^{14}$ W. Trischuk, ${ }^{164}$ B. Trocmé,${ }^{56}$ A. Trofymov, ${ }^{129}$ C. Troncon, ${ }^{66 a}$ M. Trovatelli, ${ }^{173}$ F. Trovato, ${ }^{153}$ L. Truong, ${ }^{32 b}$ M. Trzebinski, ${ }^{82}$ A. Trzupek, ${ }^{82}$ F. Tsai, ${ }^{44}$ J. C-L. Tseng, ${ }^{132}$ P. V. Tsiareshka, ${ }^{105}$ N. Tsirintanis, ${ }^{9}$ V. Tsiskaridze, ${ }^{152}$ E. G. Tskhadadze, ${ }^{156 a}$ I. I. Tsukerman, ${ }^{109}$ V. Tsulaia, ${ }^{18}$ S. Tsuno, ${ }^{79}$ D. Tsybychev, ${ }^{152}$ 
Y. Tu, ${ }^{61 \mathrm{~b}}$ A. Tudorache, ${ }^{27 \mathrm{~b}}$ V. Tudorache, ${ }^{27 \mathrm{~b}}$ T. T. Tulbure, ${ }^{27 \mathrm{a}}$ A. N. Tuna, ${ }^{57}$ S. Turchikhin, ${ }^{77}$ D. Turgeman, ${ }^{177}$ I. Turk Cakir, ${ }^{4 b, v v}$ R. Turra, ${ }^{66 a}$ P. M. Tuts, ${ }^{38}$ E. Tzovara, ${ }^{97}$ G. Ucchielli, ${ }^{23 b, 23 a}$ I. Ueda, ${ }^{79}$ M. Ughetto, ${ }^{43 a, 43 b}$ F. Ukegawa, ${ }^{166}$ G. Unal, ${ }^{35}$ A. Undrus, ${ }^{29}$ G. Unel, ${ }^{168}$ F. C. Ungaro, ${ }^{102}$ Y. Unno, ${ }^{79}$ K. Uno, ${ }^{160} \mathrm{~J}$ Urban, ${ }^{28 b}$ P. Urquijo, ${ }^{102}$ P. Urrejola, ${ }^{97}$ G. Usai, ${ }^{8}$ J. Usui, ${ }^{79}$ L. Vacavant, ${ }^{99}$ V. Vacek, ${ }^{139}$ B. Vachon, ${ }^{101}$ K. O. H. Vadla, ${ }^{131}$ A. Vaidya, ${ }^{92}$ C. Valderanis, ${ }^{112}$ E. Valdes Santurio, ${ }^{43 a, 43 b}$ M. Valente, ${ }^{52}$ S. Valentinetti, ${ }^{23 b, 23 a}$ A. Valero, ${ }^{171}$ L. Valéry, ${ }^{44}$ R. A. Vallance, ${ }^{21}$ A. Vallier, ${ }^{5}$ J. A. Valls Ferrer, ${ }^{171}$ T. R. Van Daalen, ${ }^{14}$ W. Van Den Wollenberg, ${ }^{118}$ H. Van der Graaf, ${ }^{118}$ P. Van Gemmeren, ${ }^{6}$ J. Van Nieuwkoop, ${ }^{149}$ I. Van Vulpen, ${ }^{118}$ M. C. van Woerden, ${ }^{118}$ M. Vanadia, ${ }^{71 a, 71 b}$ W. Vandelli, ${ }^{35}$ A. Vaniachine, ${ }^{163}$ P. Vankov, ${ }^{118}$ R. Vari, ${ }^{70 a}$ E. W. Varnes, ${ }^{7}$ C. Varni, ${ }^{53 b, 53 a}$ T. Varol, ${ }^{41}$ D. Varouchas, ${ }^{129}$ A. Vartapetian, ${ }^{8}$ K. E. Varvell, ${ }^{154}$ G. A. Vasquez, ${ }^{144 b}$ J. G. Vasquez, ${ }^{180}$ F. Vazeille, ${ }^{37}$ D. Vazquez Furelos, ${ }^{14}$ T. Vazquez Schroeder, ${ }^{101}$ J. Veatch, ${ }^{51}$ V. Vecchio, ${ }^{72 a, 72 b}$ L. M. Veloce, ${ }^{164}$ F. Veloso, ${ }^{137 a, 137 c}$ S. Veneziano, ${ }^{70 a}$ A. Ventura, ${ }^{65 a, 65 b}$ M. Venturi, ${ }^{173}$ N. Venturi, ${ }^{35}$ V. Vercesi, ${ }^{68 \mathrm{a}}$ M. Verducci, ${ }^{72 \mathrm{a}, 72 \mathrm{~b}}$ C. M. Vergel Infante, ${ }^{76}$ W. Verkerke, ${ }^{118}$ A. T. Vermeulen, ${ }^{118}$ J. C. Vermeulen, ${ }^{118}$ M. C. Vetterli, ${ }^{149, \mathrm{e}}$ N. Viaux Maira, ${ }^{144 \mathrm{~b}}$ O. Viazlo, ${ }^{94}$ I. Vichou, ${ }^{170, \mathrm{a}}$ T. Vickey, ${ }^{146}$ O. E. Vickey Boeriu, ${ }^{146}$ G. H. A. Viehhauser, ${ }^{132}$ S. Viel, ${ }^{18}$ L. Vigani, ${ }^{132}$ M. Villa, ${ }^{23 b, 23 a}$ M. Villaplana Perez, ${ }^{66,66 b}$ E. Vilucchi, ${ }^{49}$ M. G. Vincter, ${ }^{33}$ V. B. Vinogradov, ${ }^{77}$ A. Vishwakarma, ${ }^{44}$ C. Vittori, ${ }^{23 b, 23 a}$ I. Vivarelli, ${ }^{153}$ S. Vlachos, ${ }^{10}$ M. Vogel, ${ }^{179}$ P. Vokac, ${ }^{139}$ G. Volpi, ${ }^{14}$ S. E. von Buddenbrock, ${ }^{32 c}$ E. Von Toerne, ${ }^{24}$ V. Vorobel, ${ }^{140}$ K. Vorobev, ${ }^{110}$ M. Vos, ${ }^{171}$ J. H. Vossebeld, ${ }^{88}$ N. Vranjes, ${ }^{16}$

M. Vranjes Milosavljevic, ${ }^{16}$ V. Vrba, ${ }^{139}$ M. Vreeswijk, ${ }^{118}$ T. Šfiligoj, ${ }^{89}$ R. Vuillermet, ${ }^{35}$ I. Vukotic, $^{36}$ T. Ženiš, ${ }^{28 a}$

L. Živković, ${ }^{16}$ P. Wagner, ${ }^{24}$ W. Wagner, ${ }^{179}$ J. Wagner-Kuhr, ${ }^{112}$ H. Wahlberg, ${ }^{86}$ S. Wahrmund, ${ }^{46}$ K. Wakamiya, ${ }^{80}$ V. M. Walbrecht, ${ }^{113}$ J. Walder, ${ }^{87}$ R. Walker, ${ }^{112}$ W. Walkowiak, ${ }^{148}$ V. Wallangen, ${ }^{43 a, 43 b}$ A. M. Wang, ${ }^{57}$ C. Wang, ${ }^{58, q}$ F. Wang, ${ }^{178}$ H. Wang, ${ }^{18}$ H. Wang, ${ }^{3}$ J. Wang, ${ }^{154}$ J. Wang, ${ }^{59 b}$ P. Wang, ${ }^{41}$ Q. Wang, ${ }^{125}$ R.-J. Wang, ${ }^{133}$ R. Wang, ${ }^{58 a}$ R. Wang, ${ }^{6}$

S. M. Wang, ${ }^{155}$ W. T. Wang, ${ }^{58 \mathrm{a}}$ W. Wang, ${ }^{155, w w}$ W. X. Wang, ${ }^{58 \mathrm{a}, \mathrm{xx}}$ Y. Wang, ${ }^{58 \mathrm{a}, \mathrm{jj}}$ Z. Wang, ${ }^{58 \mathrm{c}}$ C. Wanotayaroj, ${ }^{44}$ A. Warburton, ${ }^{101}$ C. P. Ward, ${ }^{31}$ D. R. Wardrope, ${ }^{92}$ A. Washbrook, ${ }^{48}$ P. M. Watkins, ${ }^{21}$ A. T. Watson, ${ }^{21}$ M. F. Watson, ${ }^{21}$

G. Watts, ${ }^{145}$ S. Watts, ${ }^{98}$ B. M. Waugh, ${ }^{92}$ A. F. Webb, ${ }^{11}$ S. Webb, ${ }^{97}$ C. Weber, ${ }^{180}$ M. S. Weber, ${ }^{20}$ S. A. Weber, ${ }^{33}$

S. M. Weber, ${ }^{59 a}$ J. S. Webster, ${ }^{6}$ A. R. Weidberg, ${ }^{132}$ B. Weinert, ${ }^{63}$ J. Weingarten, ${ }^{51}$ M. Weirich, ${ }^{97}$ C. Weiser, ${ }^{50}$ P. S. Wells, ${ }^{35}$ T. Wenaus, ${ }^{29}$ T. Wengler, ${ }^{35}$ S. Wenig, ${ }^{35}$ N. Wermes, ${ }^{24}$ M. D. Werner, ${ }^{76}$ P. Werner, ${ }^{35}$ M. Wessels, ${ }^{59 a}$ T. D. Weston, ${ }^{20}$ K. Whalen, ${ }^{128}$ N. L. Whallon, ${ }^{145}$ A. M. Wharton, ${ }^{87}$ A. S. White, ${ }^{103}$ A. White, ${ }^{8}$ M. J. White, ${ }^{1}$ R. White, ${ }^{144 b}$ D. Whiteson, ${ }^{168}$ B. W. Whitmore, ${ }^{87}$ F. J. Wickens, ${ }^{141}$ W. Wiedenmann, ${ }^{178}$ M. Wielers, ${ }^{141}$ C. Wiglesworth, ${ }^{39}$ L. A. M. Wiik-Fuchs, ${ }^{50}$ A. Wildauer, ${ }^{113}$ F. Wilk, ${ }^{98}$ H. G. Wilkens, ${ }^{35}$ L. J. Wilkins, ${ }^{91}$ H. H. Williams, ${ }^{134}$ S. Williams, ${ }^{31}$ C. Willis, ${ }^{104}$ S. Willocq, ${ }^{100}$ J. A. Wilson, ${ }^{21}$ I. Wingerter-Seez, ${ }^{5}$ E. Winkels, ${ }^{153}$ F. Winklmeier, ${ }^{128}$ O. J. Winston, ${ }^{153}$ B. T. Winter, ${ }^{24}$ M. Wittgen, ${ }^{150}$ M. Wobisch, ${ }^{93}$ A. Wolf, ${ }^{97}$ T. M. H. Wolf, ${ }^{118}$ R. Wolff, ${ }^{99}$ M. W. Wolter, ${ }^{82}$ H. Wolters, ${ }^{137 a, 137 c}$ V. W. S. Wong, ${ }^{172}$ N. L. Woods, ${ }^{143}$ S. D. Worm, ${ }^{21}$ B. K. Wosiek, ${ }^{82}$ K. W. Woźniak, ${ }^{82}$ K. Wraight, ${ }^{55}$ M. Wu, ${ }^{36}$ S. L. Wu, ${ }^{178}$ X. Wu, ${ }^{52}$ Y. Wu, ${ }^{58 a}$ T. R. Wyatt, ${ }^{98}$ B. M. Wynne, ${ }^{48}$ S. Xella, ${ }^{39}$ Z. Xi, ${ }^{103}$ L. Xia, ${ }^{175}$ D. Xu, ${ }^{15 a}$ H. Xu, ${ }^{58 a, q}$ L. Xu, ${ }^{29}$ T. Xu, ${ }^{142}$ W. Xu, ${ }^{103}$ B. Yabsley, ${ }^{154}$ S. Yacoob, ${ }^{32 a}$ K. Yajima, ${ }^{130}$ D. P. Yallup, ${ }^{92}$ D. Yamaguchi, ${ }^{162}$ Y. Yamaguchi, ${ }^{162}$ A. Yamamoto, ${ }^{79}$ T. Yamanaka, ${ }^{160}$ F. Yamane, ${ }^{80}$ M. Yamatani, ${ }^{160}$ T. Yamazaki, ${ }^{160}$ Y. Yamazaki, ${ }^{80}$ Z. Yan, ${ }^{25}$ H. J. Yang, ${ }^{58,58 d}$ H. T. Yang, ${ }^{18}$ S. Yang, ${ }^{75}$ Y. Yang, ${ }^{160}$ Z. Yang, ${ }^{17}$ W-M. Yao, ${ }^{18}$ Y. C. Yap, ${ }^{44}$ Y. Yasu, ${ }^{79}$ E. Yatsenko, ${ }^{58 c}$ J. Ye, ${ }^{41}$ S. Ye, ${ }^{29}$ I. Yeletskikh, $^{77}$ E. Yigitbasi, ${ }^{25}$ E. Yildirim, ${ }^{97}$ K. Yorita, ${ }^{176}$ K. Yoshihara, ${ }^{134}$ C. J. S. Young, ${ }^{35}$ C. Young, ${ }^{150}$ J. Yu, ${ }^{8}$ J. Yu, ${ }^{76}$ X. Yue, ${ }^{59 a}$ S. P. Y. Yuen, ${ }^{24}$ I. Yusuff, ${ }^{31, y y}$ B. Zabinski, ${ }^{82}$ G. Zacharis, ${ }^{10}$ E. Zaffaroni, ${ }^{52}$ R. Zaidan, ${ }^{14}$ A. M. Zaitsev, ${ }^{121, n n}$ N. Zakharchuk, ${ }^{44}$ J. Zalieckas, ${ }^{17}$ S. Zambito, ${ }^{57}$ D. Zanzi, ${ }^{35}$ D. R. Zaripovas, ${ }^{55}$ S. V. Zeißner, ${ }^{45}$ C. Zeitnitz, ${ }^{179}$ G. Zemaityte, ${ }^{132}$ J. C. Zeng, ${ }^{170}$ Q. Zeng, ${ }^{150}$ O. Zenin, ${ }^{121}$ D. Zerwas, ${ }^{129}$ M. Zgubič, ${ }^{132}$ D. F. Zhang, ${ }^{58 \mathrm{~b}}$ D. Zhang, ${ }^{103}$ F. Zhang, ${ }^{178}$ G. Zhang, ${ }^{58 \mathrm{a}, \mathrm{xx}}$ H. Zhang, ${ }^{15 \mathrm{c}}$ J. Zhang, ${ }^{6}$ L. Zhang, ${ }^{50}$ L. Zhang, ${ }^{58 \mathrm{a}}$ M. Zhang, ${ }^{170}$ P. Zhang, ${ }^{15 \mathrm{c}}$ R. Zhang, ${ }^{58 \mathrm{a}, \mathrm{q}}$ R. Zhang, ${ }^{24} \mathrm{X}$. Zhang, ${ }^{58 \mathrm{~b}} \mathrm{Y}$. Zhang, ${ }^{15 \mathrm{~d}} \mathrm{Z}$. Zhang, ${ }^{129}$ P. Zhao, ${ }^{47}$ X. Zhao, ${ }^{41}$ Y. Zhao, ${ }^{58 b, 129, z}$ Z. Zhao, ${ }^{58 a}$ A. Zhemchugov, ${ }^{77}$ B. Zhou, ${ }^{103}$ C. Zhou, ${ }^{178}$ L. Zhou, ${ }^{41}$ M. S. Zhou, ${ }^{15 d}$ M. Zhou, ${ }^{152}$ N. Zhou, ${ }^{58 \mathrm{c}}$ Y. Zhou, ${ }^{7}$ C. G. Zhu, ${ }^{58 b}$ H. L. Zhu, ${ }^{58 \mathrm{a}}$ H. Zhu, ${ }^{15 \mathrm{a}}$ J. Zhu, ${ }^{103}$ Y. Zhu, ${ }^{58 \mathrm{a}}$ X. Zhuang, ${ }^{15 \mathrm{a}}$ K. Zhukov, ${ }^{108}$ V. Zhulanov, ${ }^{120 b, 120 a}$ A. Zibell, ${ }^{174}$ D. Zieminska, ${ }^{63}$ N. I. Zimine, ${ }^{77}$ S. Zimmermann, ${ }^{50}$ Z. Zinonos, ${ }^{113}$ M. Zinser, ${ }^{97}$

M. Ziolkowski, ${ }^{148}$ G. Zobernig, ${ }^{178}$ A. Zoccoli, ${ }^{23 b, 23 a}$ K. Zoch, ${ }^{51}$ T. G. Zorbas, ${ }^{146}$ R. Zou, ${ }^{36}$ M. Zur Nedden, ${ }^{19}$ and L. Zwalinski ${ }^{35}$

(ATLAS Collaboration)

\footnotetext{
${ }^{1}$ Department of Physics, University of Adelaide, Adelaide, Australia

${ }^{2}$ Physics Department, SUNY Albany, Albany, New York, USA

${ }^{3}$ Department of Physics, University of Alberta, Edmonton, Alberta, Canada
} 
${ }^{4 a}$ Department of Physics, Ankara University, Ankara, Turkey

${ }^{4 \mathrm{~b}}$ Istanbul Aydin University, Istanbul, Turkey

${ }^{4 c}$ Division of Physics, TOBB University of Economics and Technology, Ankara, Turkey

${ }^{5}$ LAPP, Université Grenoble Alpes, Université Savoie Mont Blanc, CNRS/IN2P3, Annecy, France

${ }^{6}$ High Energy Physics Division, Argonne National Laboratory, Argonne, Illinois, USA

${ }^{7}$ Department of Physics, University of Arizona, Tucson, Arizona, USA

${ }^{8}$ Department of Physics, University of Texas at Arlington, Arlington, Texas, USA

${ }^{9}$ Physics Department, National and Kapodistrian University of Athens, Athens, Greece

${ }^{10}$ Physics Department, National Technical University of Athens, Zografou, Greece

${ }^{11}$ Department of Physics, University of Texas at Austin, Austin, Texas, USA

${ }^{12 \mathrm{a}}$ Bahcesehir University, Faculty of Engineering and Natural Sciences, Istanbul, Turkey

${ }^{12 \mathrm{~b}}$ Istanbul Bilgi University, Faculty of Engineering and Natural Sciences, Istanbul, Turkey

${ }^{12 \mathrm{c}}$ Department of Physics, Bogazici University, Istanbul, Turkey

${ }^{12 \mathrm{~d}}$ Department of Physics Engineering, Gaziantep University, Gaziantep, Turkey

${ }^{13}$ Institute of Physics, Azerbaijan Academy of Sciences, Baku, Azerbaijan

${ }^{14}$ Institut de Física d'Altes Energies (IFAE), Barcelona Institute of Science and Technology, Barcelona, Spain

${ }^{15 a}$ Institute of High Energy Physics, Chinese Academy of Sciences, Beijing, China

${ }^{15 \mathrm{~b}}$ Physics Department, Tsinghua University, Beijing, China

${ }^{15 c}$ Department of Physics, Nanjing University, Nanjing, China

${ }^{15 \mathrm{~d}}$ University of Chinese Academy of Science (UCAS), Beijing, China

${ }^{16}$ Institute of Physics, University of Belgrade, Belgrade, Serbia

${ }^{17}$ Department for Physics and Technology, University of Bergen, Bergen, Norway

${ }^{18}$ Physics Division, Lawrence Berkeley National Laboratory and University of California, Berkeley, California, USA

${ }^{19}$ Institut für Physik, Humboldt Universität zu Berlin, Berlin, Germany

${ }^{20}$ Albert Einstein Center for Fundamental Physics and Laboratory for High Energy Physics, University of Bern, Bern, Switzerland

${ }^{21}$ School of Physics and Astronomy, University of Birmingham, Birmingham, United Kingdom

${ }^{22}$ Centro de Investigaciónes, Universidad Antonio Nariño, Bogota, Colombia

${ }^{23 a}$ Dipartimento di Fisica e Astronomia, Università di Bologna, Bologna, Italy

${ }^{23 \mathrm{~b}}$ INFN Sezione di Bologna, Italy

${ }^{24}$ Physikalisches Institut, Universität Bonn, Bonn, Germany

${ }^{25}$ Department of Physics, Boston University, Boston, Massachusetts, USA

${ }^{26}$ Department of Physics, Brandeis University, Waltham, Massachusetts, USA

${ }^{27}$ Transilvania University of Brasov, Brasov, Romania

${ }^{27 \mathrm{~b}}$ Horia Hulubei National Institute of Physics and Nuclear Engineering, Bucharest, Romania

${ }^{27 \mathrm{c}}$ Department of Physics, Alexandru Ioan Cuza University of Iasi, Iasi, Romania

${ }^{27 \mathrm{~d}}$ National Institute for Research and Development of Isotopic and Molecular Technologies, Physics Department, Cluj-Napoca, Romania

${ }^{27 \mathrm{e}}$ University Politehnica Bucharest, Bucharest, Romania

${ }^{27 \mathrm{f}}$ West University in Timisoara, Timisoara, Romania

${ }^{28 a}$ Faculty of Mathematics, Physics and Informatics, Comenius University, Bratislava, Slovak Republic

${ }^{28 \mathrm{~b}}$ Department of Subnuclear Physics, Institute of Experimental Physics of the Slovak Academy of Sciences,

Kosice, Slovak Republic

${ }^{29}$ Physics Department, Brookhaven National Laboratory, Upton, New York, USA

${ }^{30}$ Departamento de Física, Universidad de Buenos Aires, Buenos Aires, Argentina

${ }^{31}$ Cavendish Laboratory, University of Cambridge, Cambridge, United Kingdom

${ }^{32 a}$ Department of Physics, University of Cape Town, Cape Town, South Africa

${ }^{32 \mathrm{~b}}$ Department of Mechanical Engineering Science, University of Johannesburg, Johannesburg, South Africa

${ }^{32 \mathrm{c}}$ School of Physics, University of the Witwatersrand, Johannesburg, South Africa

${ }^{33}$ Department of Physics, Carleton University, Ottawa, Ontario, Canada

${ }^{34 \mathrm{a}}$ Faculté des Sciences Ain Chock, Réseau Universitaire de Physique des Hautes Energies-Université Hassan II, Casablanca, Morocco

${ }^{34 \mathrm{~b}}$ Centre National de l'Energie des Sciences Techniques Nucleaires (CNESTEN), Rabat, Morocco

${ }^{34 \mathrm{c}}$ Faculté des Sciences Semlalia, Université Cadi Ayyad, LPHEA-Marrakech, Morocco

${ }^{34 \mathrm{~d}}$ Faculté des Sciences, Université Mohamed Premier and LPTPM, Oujda, Morocco

${ }^{34 \mathrm{e}}$ Faculté des sciences, Université Mohammed V, Rabat, Morocco

${ }^{35}$ CERN, Geneva, Switzerland 
${ }^{36}$ Enrico Fermi Institute, University of Chicago, Chicago, Illinois, USA

${ }^{37}$ LPC, Université Clermont Auvergne, CNRS/IN2P3, Clermont-Ferrand, France

${ }^{38}$ Nevis Laboratory, Columbia University, Irvington, New York, USA

${ }^{39}$ Niels Bohr Institute, University of Copenhagen, Copenhagen, Denmark

${ }^{40 a}$ Dipartimento di Fisica, Università della Calabria, Rende, Italy

${ }^{40 \mathrm{~b}}$ INFN Gruppo Collegato di Cosenza, Laboratori Nazionali di Frascati, Italy

${ }^{41}$ Physics Department, Southern Methodist University, Dallas, Texas, USA

${ }^{42}$ Physics Department, University of Texas at Dallas, Richardson, Texas, USA

${ }^{43}$ Department of Physics, Stockholm University, Sweden

${ }^{43 \mathrm{~b}}$ Oskar Klein Centre, Stockholm, Sweden

${ }^{44}$ Deutsches Elektronen-Synchrotron DESY, Hamburg and Zeuthen, Germany

${ }^{45}$ Lehrstuhl für Experimentelle Physik IV, Technische Universität Dortmund, Dortmund, Germany

${ }^{46}$ Institut für Kern- und Teilchenphysik, Technische Universität Dresden, Dresden, Germany

${ }^{47}$ Department of Physics, Duke University, Durham, North Carolina, USA

${ }^{48}$ SUPA-School of Physics and Astronomy, University of Edinburgh, Edinburgh, United Kingdom

${ }^{49}$ INFN e Laboratori Nazionali di Frascati, Frascati, Italy

${ }^{50}$ Physikalisches Institut, Albert-Ludwigs-Universität Freiburg, Freiburg, Germany

${ }^{51}$ II. Physikalisches Institut, Georg-August-Universität Göttingen, Göttingen, Germany

${ }^{52}$ Département de Physique Nucléaire et Corpusculaire, Université de Genève, Genève, Switzerland

${ }^{53}$ Dipartimento di Fisica, Università di Genova, Genova, Italy

${ }^{53 \mathrm{~b}}$ INFN Sezione di Genova, Italy

${ }^{54}$ II. Physikalisches Institut, Justus-Liebig-Universität Giessen, Giessen, Germany

${ }^{55}$ SUPA-School of Physics and Astronomy, University of Glasgow, Glasgow, United Kingdom

${ }^{56}$ LPSC, Université Grenoble Alpes, CNRS/IN2P3, Grenoble INP, Grenoble, France

${ }^{57}$ Laboratory for Particle Physics and Cosmology, Harvard University, Cambridge, Massachusetts, USA

${ }^{58 \mathrm{a}}$ Department of Modern Physics and State Key Laboratory of Particle Detection and Electronics, University of Science and Technology of China, Hefei, China

${ }^{58 \mathrm{~b}}$ Institute of Frontier and Interdisciplinary Science and Key Laboratory of Particle Physics and

Particle Irradiation (MOE), Shandong University, Qingdao, China

${ }^{58 \mathrm{c}}$ School of Physics and Astronomy, Shanghai Jiao Tong University, KLPPAC-MoE, SKLPPC, Shanghai, China

${ }^{58 \mathrm{~d}}$ Tsung-Dao Lee Institute, Shanghai, China

${ }^{59 a}$ Kirchhoff-Institut für Physik, Ruprecht-Karls-Universität Heidelberg, Heidelberg, Germany

${ }^{59 \mathrm{~b}}$ Physikalisches Institut, Ruprecht-Karls-Universität Heidelberg, Heidelberg, Germany

${ }^{60}$ Faculty of Applied Information Science, Hiroshima Institute of Technology, Hiroshima, Japan

${ }^{61 a}$ Department of Physics, Chinese University of Hong Kong, Shatin, N.T., Hong Kong, China

${ }^{61 \mathrm{~b}}$ Department of Physics, University of Hong Kong, Hong Kong, China

${ }^{61 \mathrm{c} D e p a r t m e n t}$ of Physics and Institute for Advanced Study, Hong Kong University of Science and

Technology, Clear Water Bay, Kowloon, Hong Kong, China

${ }^{62}$ Department of Physics, National Tsing Hua University, Hsinchu, Taiwan

${ }^{63}$ Department of Physics, Indiana University, Bloomington, Indiana, USA

${ }^{64 a}$ INFN Gruppo Collegato di Udine, Sezione di Trieste, Udine, Italy

${ }^{64 \mathrm{~b}}$ ICTP, Trieste, Italy

${ }^{64 \mathrm{c}}$ Dipartimento di Chimica, Fisica e Ambiente, Università di Udine, Udine, Italy

${ }^{65}$ INFN Sezione di Lecce, Italy

${ }^{65 \mathrm{~b}}$ Dipartimento di Matematica e Fisica, Università del Salento, Lecce, Italy

${ }^{66 a}$ INFN Sezione di Milano, Italy

${ }^{66 \mathrm{~b}}$ Dipartimento di Fisica, Università di Milano, Milano, Italy

${ }^{67 a}$ INFN Sezione di Napoli, Italy

${ }^{67 \mathrm{~b}}$ Dipartimento di Fisica, Università di Napoli, Napoli, Italy

${ }^{68 \mathrm{a}}$ INFN Sezione di Pavia, Italy

${ }^{68 \mathrm{~b}}$ Dipartimento di Fisica, Università di Pavia, Pavia, Italy

${ }^{69 a}$ INFN Sezione di Pisa, Italy

${ }^{69 \mathrm{~b}}$ Dipartimento di Fisica E. Fermi, Università di Pisa, Pisa, Italy

${ }^{70 a}$ INFN Sezione di Roma, Italy

${ }^{70 \mathrm{~b}}$ Dipartimento di Fisica, Sapienza Università di Roma, Roma, Italy

${ }^{71 a}$ INFN Sezione di Roma Tor Vergata, Italy

${ }^{71 b}$ Dipartimento di Fisica, Università di Roma Tor Vergata, Roma, Italy

${ }^{72 \mathrm{a}}$ INFN Sezione di Roma Tre, Italy

${ }^{72 b}$ Dipartimento di Matematica e Fisica, Università Roma Tre, Roma, Italy 


\author{
${ }^{73 a}$ INFN-TIFPA, Italy \\ ${ }^{73 \mathrm{~b}}$ Università degli Studi di Trento, Trento, Italy \\ ${ }^{74}$ Institut für Astro- und Teilchenphysik, Leopold-Franzens-Universität, Innsbruck, Austria \\ ${ }^{75}$ University of Iowa, Iowa City, Iowa, USA \\ ${ }^{76}$ Department of Physics and Astronomy, Iowa State University, Ames, Iowa, USA \\ ${ }^{77}$ Joint Institute for Nuclear Research, Dubna, Russia \\ ${ }^{78 a}$ Departamento de Engenharia Elétrica, Universidade Federal de Juiz de Fora (UFJF), \\ Juiz de Fora, Brazil \\ ${ }^{78 b}$ Universidade Federal do Rio De Janeiro COPPE/EE/IF, Rio de Janeiro, Brazil \\ ${ }^{78 c}$ Universidade Federal de São João del Rei (UFSJ), São João del Rei, Brazil \\ ${ }^{78 d}$ Instituto de Física, Universidade de São Paulo, São Paulo, Brazil \\ ${ }^{79}$ KEK, High Energy Accelerator Research Organization, Tsukuba, Japan \\ ${ }^{80}$ Graduate School of Science, Kobe University, Kobe, Japan \\ ${ }^{81 \mathrm{a}}$ AGH University of Science and Technology, Faculty of Physics and Applied Computer Science, \\ Krakow, Poland \\ ${ }^{81 \mathrm{~b}}$ Marian Smoluchowski Institute of Physics, Jagiellonian University, Krakow, Poland \\ ${ }^{82}$ Institute of Nuclear Physics Polish Academy of Sciences, Krakow, Poland \\ ${ }^{83}$ Faculty of Science, Kyoto University, Kyoto, Japan \\ ${ }^{84}$ Kyoto University of Education, Kyoto, Japan \\ ${ }^{85}$ Research Center for Advanced Particle Physics and Department of Physics, Kyushu University, \\ Fukuoka, Japan \\ ${ }^{86}$ Instituto de Física La Plata, Universidad Nacional de La Plata and CONICET, La Plata, Argentina \\ ${ }^{87}$ Physics Department, Lancaster University, Lancaster, United Kingdom \\ ${ }^{88}$ Oliver Lodge Laboratory, University of Liverpool, Liverpool, United Kingdom \\ ${ }^{89}$ Department of Experimental Particle Physics, Jožef Stefan Institute and Department of Physics, \\ University of Ljubljana, Ljubljana, Slovenia \\ ${ }^{90}$ School of Physics and Astronomy, Queen Mary University of London, London, United Kingdom \\ ${ }^{91}$ Department of Physics, Royal Holloway University of London, Egham, United Kingdom \\ ${ }^{92}$ Department of Physics and Astronomy, University College London, London, United Kingdom \\ ${ }^{93}$ Louisiana Tech University, Ruston, Louisiana, USA \\ ${ }^{94}$ Fysiska institutionen, Lunds universitet, Lund, Sweden \\ ${ }^{95}$ Centre de Calcul de l'Institut National de Physique Nucléaire et de Physique des Particules (IN2P3), \\ Villeurbanne, France \\ ${ }^{96}$ Departamento de Física Teorica C-15 and CIAFF, Universidad Autónoma de Madrid, Madrid, Spain \\ ${ }^{97}$ Institut für Physik, Universität Mainz, Mainz, Germany \\ ${ }^{98}$ School of Physics and Astronomy, University of Manchester, Manchester, United Kingdom \\ ${ }^{99} \mathrm{CPPM}$, Aix-Marseille Université, CNRS/IN2P3, Marseille, France \\ ${ }^{100}$ Department of Physics, University of Massachusetts, Amherst, Massachusetts, USA \\ ${ }^{101}$ Department of Physics, McGill University, Montreal, Québec, Canada \\ ${ }^{102}$ School of Physics, University of Melbourne, Victoria, Australia \\ ${ }^{103}$ Department of Physics, University of Michigan, Ann Arbor, Michigan, USA \\ ${ }^{104}$ Department of Physics and Astronomy, Michigan State University, East Lansing, Michigan, USA \\ ${ }^{105}$ B. I. Stepanov Institute of Physics, National Academy of Sciences of Belarus, Minsk, Belarus \\ ${ }^{106}$ Research Institute for Nuclear Problems of Byelorussian State University, Minsk, Belarus \\ ${ }^{107}$ Group of Particle Physics, University of Montreal, Montreal, Québec, Canada \\ ${ }^{108}$ P. N. Lebedev Physical Institute of the Russian Academy of Sciences, Moscow, Russia \\ ${ }^{109}$ Institute for Theoretical and Experimental Physics (ITEP), Moscow, Russia \\ ${ }^{110}$ National Research Nuclear University MEPhI, Moscow, Russia \\ ${ }^{111}$ D. V. Skobeltsyn Institute of Nuclear Physics, M. V. Lomonosov Moscow State University, \\ Moscow, Russia \\ ${ }^{112}$ Fakultät für Physik, Ludwig-Maximilians-Universität München, München, Germany \\ ${ }^{113}$ Max-Planck-Institut für Physik (Werner-Heisenberg-Institut), München, Germany \\ ${ }^{114}$ Nagasaki Institute of Applied Science, Nagasaki, Japan \\ ${ }^{115}$ Graduate School of Science and Kobayashi-Maskawa Institute, Nagoya University, Nagoya, Japan \\ ${ }^{116}$ Department of Physics and Astronomy, University of New Mexico, Albuquerque, New Mexico, USA \\ ${ }^{117}$ Institute for Mathematics, Astrophysics and Particle Physics, Radboud University Nijmegen/Nikhef, \\ Nijmegen, Netherlands \\ ${ }^{118}$ Nikhef National Institute for Subatomic Physics and University of Amsterdam, Amsterdam, Netherlands \\ ${ }^{119}$ Department of Physics, Northern Illinois University, DeKalb, Illinois, USA \\ ${ }^{120 a}$ Budker Institute of Nuclear Physics, SB RAS, Novosibirsk, Russia
}




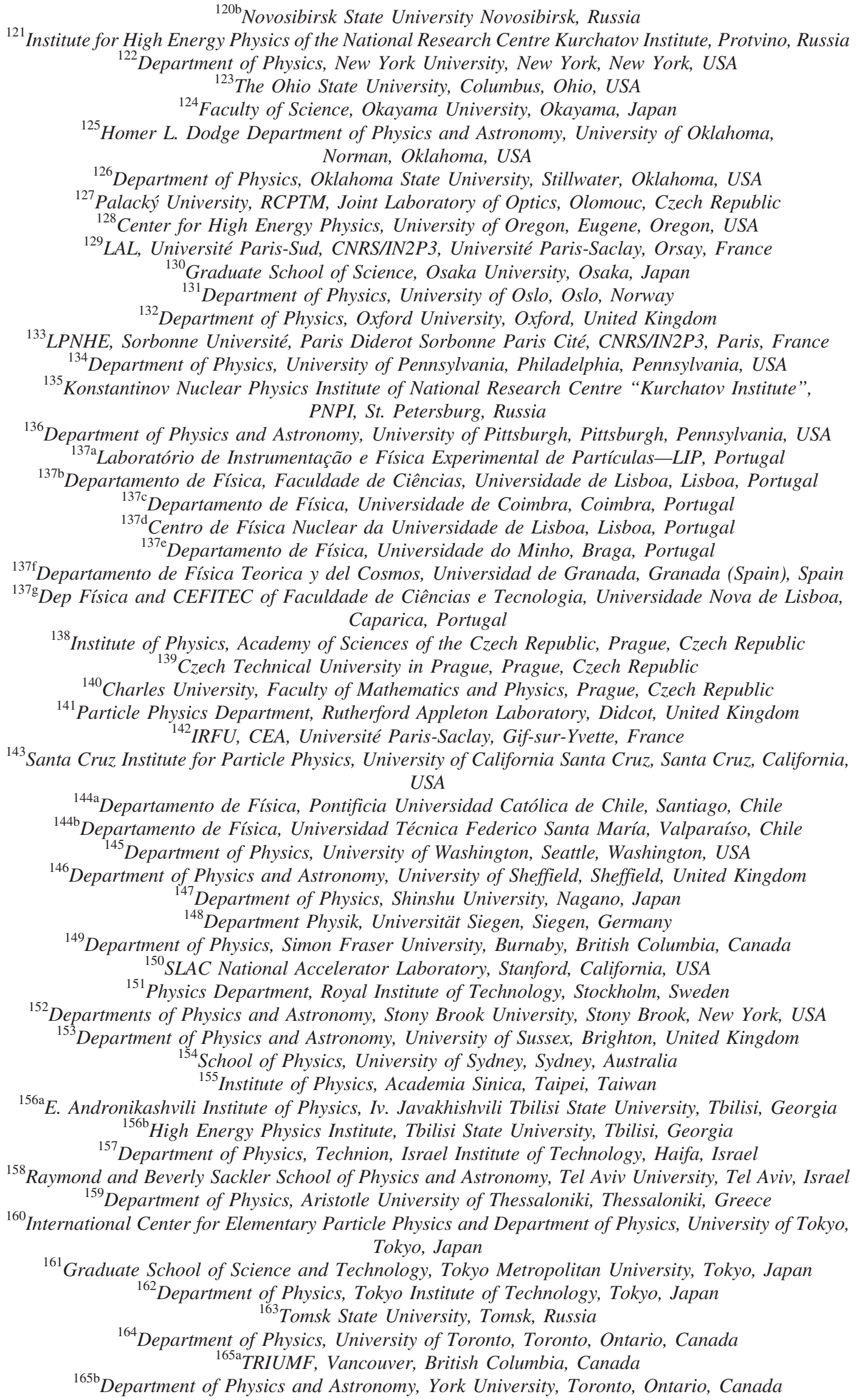


${ }^{166}$ Division of Physics and Tomonaga Center for the History of the Universe, Faculty of Pure and Applied Sciences, University of Tsukuba, Tsukuba, Japan

${ }^{167}$ Department of Physics and Astronomy, Tufts University, Medford, Massachusetts, USA

${ }^{168}$ Department of Physics and Astronomy, University of California Irvine, Irvine, California, USA

${ }^{169}$ Department of Physics and Astronomy, University of Uppsala, Uppsala, Sweden

${ }^{170}$ Department of Physics, University of Illinois, Urbana, Illinois, USA

${ }^{171}$ Instituto de Física Corpuscular (IFIC), Centro Mixto Universidad de Valencia-CSIC, Valencia, Spain

${ }^{172}$ Department of Physics, University of British Columbia, Vancouver, British Columbia, Canada

${ }^{173}$ Department of Physics and Astronomy, University of Victoria, Victoria, British Columbia, Canada

${ }^{174}$ Fakultät für Physik und Astronomie, Julius-Maximilians-Universität Würzburg, Würzburg, Germany

${ }^{175}$ Department of Physics, University of Warwick, Coventry, United Kingdom

${ }^{176}$ Waseda University, Tokyo, Japan

${ }^{177}$ Department of Particle Physics, Weizmann Institute of Science, Rehovot, Israel

${ }^{178}$ Department of Physics, University of Wisconsin, Madison, Wisconsin, USA

${ }^{179}$ Fakultät für Mathematik und Naturwissenschaften, Fachgruppe Physik, Bergische Universität Wuppertal, Wuppertal, Germany

${ }^{180}$ Department of Physics, Yale University, New Haven, Connecticut, USA

${ }^{181}$ Yerevan Physics Institute, Yerevan, Armenia

${ }^{\mathrm{a}}$ Deceased.

${ }^{\mathrm{b}}$ Also at Department of Physics, King's College London, London, United Kingdom.

${ }^{\mathrm{c}}$ Also at Istanbul University, Dept. of Physics, Istanbul, Turkey.

${ }^{\mathrm{d}}$ Also at Institute of Physics, Azerbaijan Academy of Sciences, Baku, Azerbaijan.

${ }^{\mathrm{e}}$ Also at TRIUMF, Vancouver, British Columbia, Canada.

${ }_{\mathrm{f}}^{\mathrm{f}}$ Also at Department of Physics and Astronomy, University of Louisville, Louisville, Kentucky, USA.

${ }^{\mathrm{g}}$ Also at Department of Physics, California State University, Fresno, California, USA.

${ }^{\mathrm{h}}$ Also at Department of Physics, University of Fribourg, Fribourg, Switzerland.

${ }^{\mathrm{i}}$ Also at Departament de Fisica de la Universitat Autonoma de Barcelona, Barcelona, Spain.

${ }^{\mathrm{j}}$ Also at Tomsk State University, Tomsk, and Moscow Institute of Physics and Technology State University, Dolgoprudny, Russia.

${ }^{\mathrm{k}}$ Also at The Collaborative Innovation Center of Quantum Matter (CICQM), Beijing, China.

${ }^{1}$ Also at Universita di Napoli Parthenope, Napoli, Italy.

${ }^{\mathrm{m}}$ Also at Institute of Particle Physics (IPP), Canada.

${ }^{\mathrm{n}}$ Also at II. Physikalisches Institut, Georg-August-Universität Göttingen, Göttingen, Germany.

${ }^{\circ}$ Also at Dipartimento di Fisica E. Fermi, Università di Pisa, Pisa, Italy.

${ }^{\mathrm{p}}$ Also at Horia Hulubei National Institute of Physics and Nuclear Engineering, Bucharest, Romania.

${ }^{\mathrm{q}}$ Also at CPPM, Aix-Marseille Université, CNRS/IN2P3, Marseille, France.

${ }^{\mathrm{r}}$ Also at Department of Physics, St. Petersburg State Polytechnical University, St. Petersburg, Russia.

${ }^{\mathrm{s}}$ Also at Borough of Manhattan Community College, City University of New York, New York, USA.

${ }^{t}$ Also at Department of Financial and Management Engineering, University of the Aegean, Chios, Greece.

${ }^{\mathrm{u}}$ Also at Centre for High Performance Computing, CSIR Campus, Rosebank, Cape Town, South Africa.

${ }^{v}$ Also at Louisiana Tech University, Ruston, Louisiana, USA.

${ }^{\mathrm{w}}$ Also at California State University, East Bay, USA.

${ }^{\mathrm{x}}$ Also at Institucio Catalana de Recerca i Estudis Avancats, ICREA, Barcelona, Spain.

${ }^{y}$ Also at Department of Physics, University of Michigan, Ann Arbor, Michigan, USA.

${ }^{\mathrm{z}}$ Also at LAL, Université Paris-Sud, CNRS/IN2P3, Université Paris-Saclay, Orsay, France.

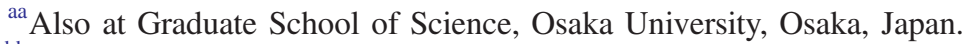

${ }^{\mathrm{bb}}$ Also at Physikalisches Institut, Albert-Ludwigs-Universität Freiburg, Freiburg, Germany.

${ }^{c c}$ Also at Institute for Mathematics, Astrophysics and Particle Physics, Radboud University Nijmegen/Nikhef, Nijmegen, Netherlands.

${ }^{\mathrm{dd}}$ Also at Near East University, Nicosia, North Cyprus, Mersin, Turkey.

${ }^{e e}$ Also at Institute of Theoretical Physics, Ilia State University, Tbilisi, Georgia.

${ }^{\mathrm{ff}}$ Also at CERN, Geneva, Switzerland.

gg Also at Department of Physics, Stanford University, USA.

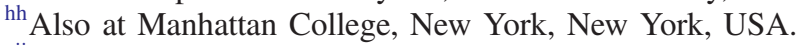

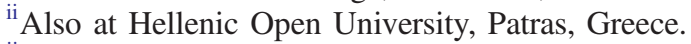

${ }^{j j}$ Also at LPNHE, Sorbonne Université, Paris Diderot Sorbonne Paris Cité, CNRS/IN2P3, Paris, France.

${ }^{\mathrm{k}}$ Also at The City College of New York, New York, New York, USA.

${ }^{11}$ Also at Departamento de Física Teorica y del Cosmos, Universidad de Granada, Granada (Spain), Spain.

${ }^{\mathrm{mm}}$ Also at Department of Physics, California State University, Sacramento, California, USA.

${ }^{n n}$ Also at Moscow Institute of Physics and Technology State University, Dolgoprudny, Russia. 
${ }^{\text {oo }}$ Also at Département de Physique Nucléaire et Corpusculaire, Université de Genève, Genève, Switzerland.

${ }^{\mathrm{pp}}$ Also at Department of Physics and Astronomy, University of Sheffield, Sheffield, United Kingdom.

${ }^{\mathrm{qq}}$ Also at School of Physics, Sun Yat-sen University, Guangzhou, China.

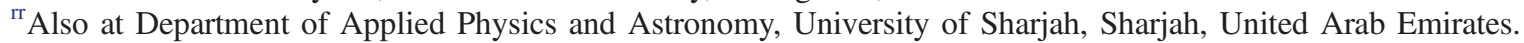

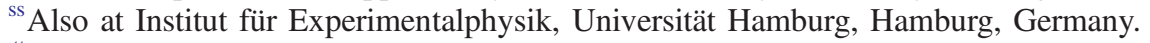

${ }^{\text {tt} A l s o ~ a t ~ N a t i o n a l ~ R e s e a r c h ~ N u c l e a r ~ U n i v e r s i t y ~ M E P h I, ~ M o s c o w, ~ R u s s i a . ~}$

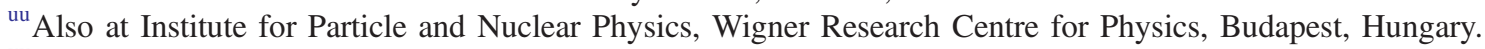

${ }^{\mathrm{vv}}$ Also at Giresun University, Faculty of Engineering, Giresun, Turkey.

${ }^{w w}$ Also at Department of Physics, Nanjing University, Nanjing, China.

${ }^{\mathrm{xx}}$ Also at Institute of Physics, Academia Sinica, Taipei, Taiwan.

${ }^{y y}$ Also at Department of Physics, University of Malaya, Kuala Lumpur, Malaysia. 